Aus der Poliklinik für Kieferorthopädie

(Prof. Dr. med. dent. P. Meyer-Marcotty)

im Zentrum Zahn-, Mund- und Kieferheilkunde

der Medizinischen Fakultät der Universität Göttingen

\title{
Pathologische Zahnwanderung der Frontzähne im parodontal geschädigten Gebiss
}

INAUGURAL-DISSERTATION

zur Erlangung des Doktorgrades

für Zahnmedizin

der Medizinischen Fakultät der

Georg-August-Universität zu Göttingen

vorgelegt von

Larissa Knocks

aus

Minden

Göttingen 2020 
Dekan:

Betreuungsausschuss

Betreuer:

Ko-Betreuerin:

\section{Prüfungskommission}

Referent:

Ko-Referentin:

Drittreferent:
Prof. Dr. med. W. Brück

Prof. Dr. med. dent. P. Meyer-Marcotty

Prof. Dr. med. dent. A. Wiegand

Prof. Dr. med. dent. P. Meyer-Marcotty

Prof. Dr. med. dent. A. Wiegand

Prof. Dr. med. M. Oppermann

Datum der mündlichen Prüfung: 08.06.2021 
Hiermit erkläre ich, die Dissertation mit dem Titel „Pathologische Zahnwanderung der Frontzähne im parodontal geschädigten Gebiss“ eigenständig angefertigt und keine anderen als die von mir angegebenen Quellen und Hilfsmittel verwendet zu haben.

Göttingen, den .............. 
Die Daten, auf denen die vorliegende Arbeit basiert, wurden teilweise publiziert:

„Vortrag im Rahmen der Deutschen Gesellschaft für Kieferorthopädie e.V., Bonn, 11.10.-14.10.2017“

„Posterpräsentation im Rahmen des european orthodontic society congress, Nizza, 17.06.-22.06.2019“ 


\section{Inhaltsverzeichnis}

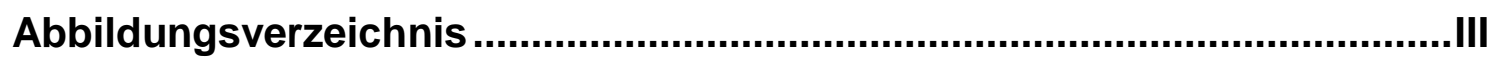

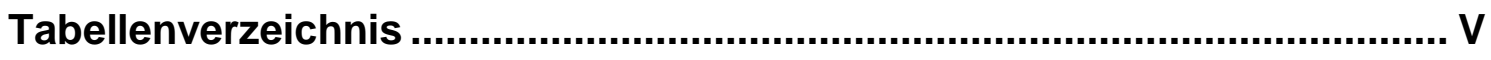

Abkürzungsverzeichnis ........................................................................ VI

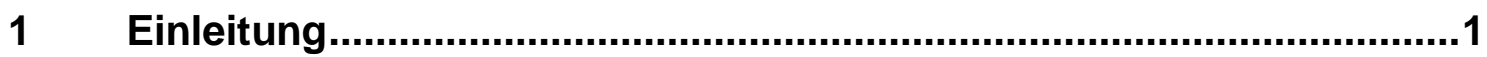

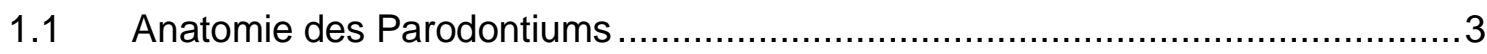

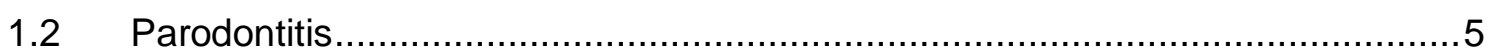

1.2.1 Ätiologie und Pathogenese der Parodontalerkrankungen ................................5

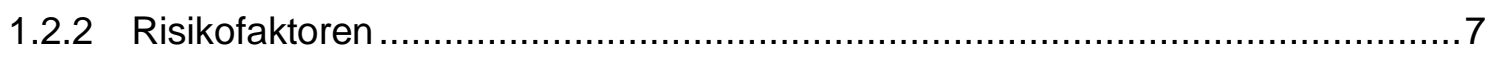

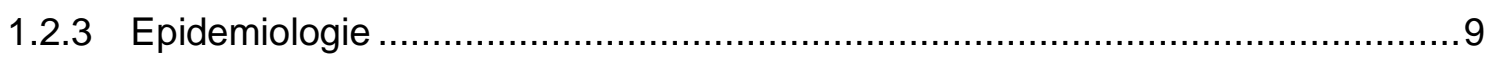

1.2.4 Definition und Klassifikation der Parodontalerkrankungen...............................10

1.3 Zusammenhang zwischen Parodontitis und pathologischen

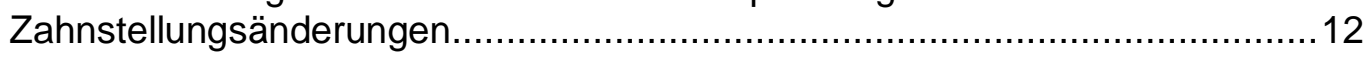

1.3.1 Kombinierte kieferorthopädisch-parodontale Therapie ...................................13

1.3.2 Mehrwert der kieferorthopädischen Therapie im parodontal geschwächten

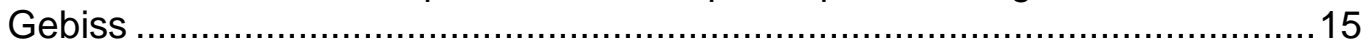

1.3.3 Beispiel eines Patientenfalls aus der Poliklinik für Kieferorthopädie der

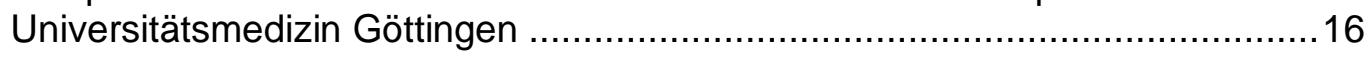

1.3.4 Intrusion parodontal kompromittierter Frontzähne ......................................19

1.4 Dreidimensionale Generierung von Oberflächendaten in der Zahnheilkunde ...20

1.4.1 Pathologische Weichteilveränderungen aufgrund von Zahnfehlstellungen.......21

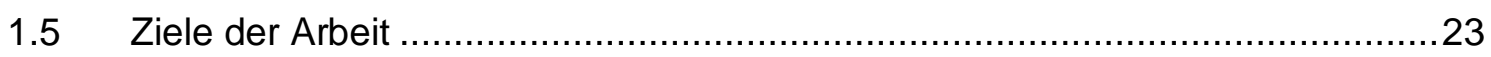

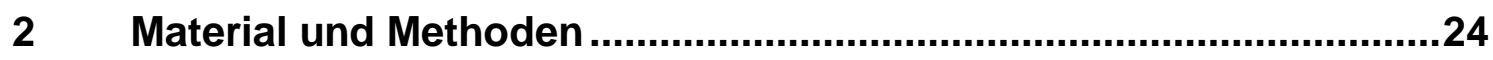

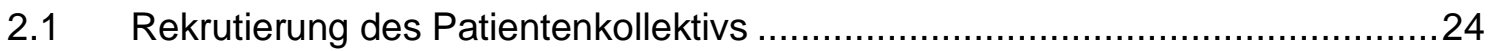

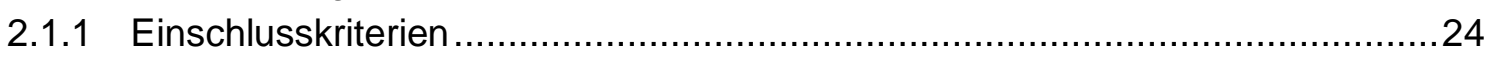

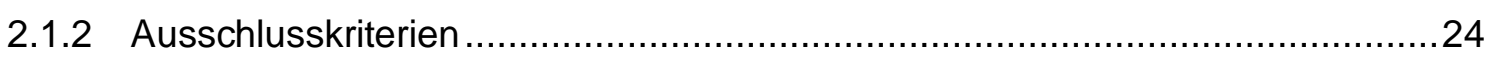

2.2 Datenerhebung - klinisch parodontale Parameter ………………................25

2.2.1 Definition der Patientengruppen..................................................................26

2.3 Modellvermessung - orthodontische Parameter ……….................................28

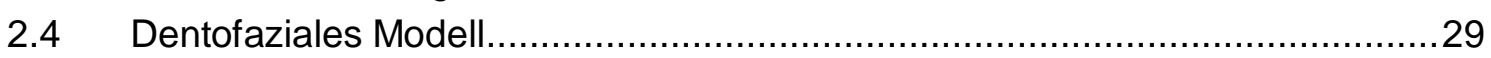

2.4.1 Erstellung des virtuellen Patientenmodells .................................................29

2.4.2 Erstellung des dreidimensionalen Patientenmodells .......................................31

2.4.3 Vermessung des dreidimensionalen Patientenmodells ....................................34

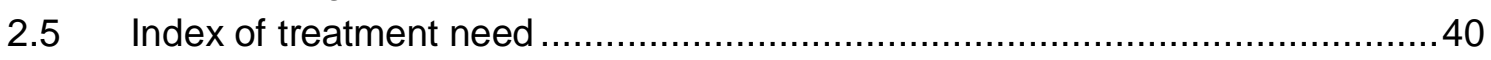

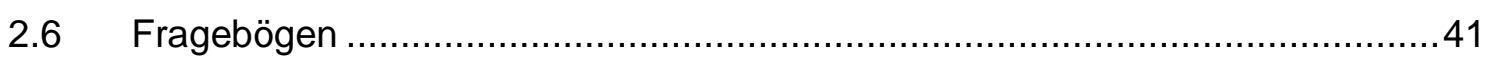

2.6.1 Fragebogen zum patientenindividuellen kierferorthopädischen

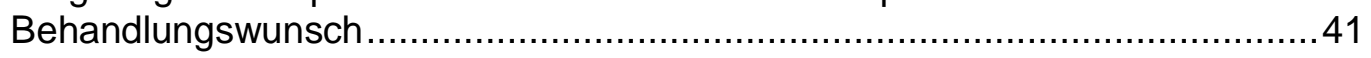

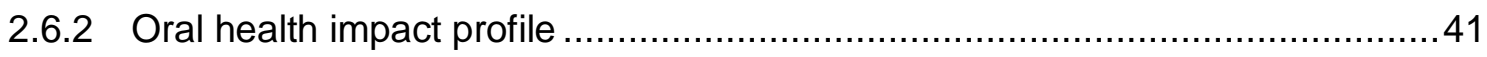

2.6.3 General / geriatric oral health assessment index.........................................42

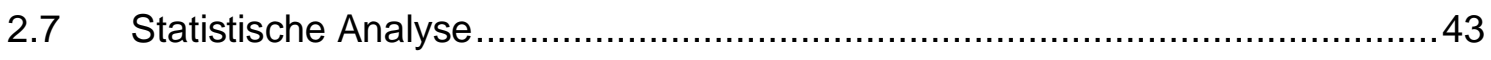

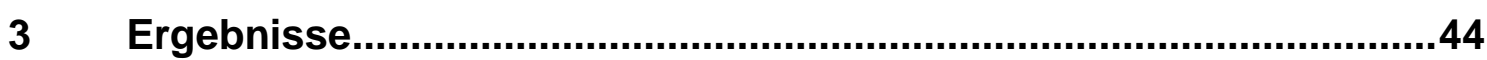




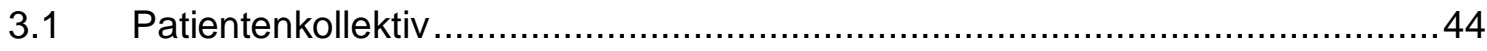

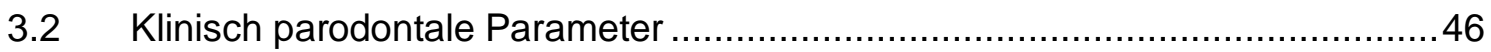

3.2.1 Parameter der Gruppeneinteilung .................................................... 46

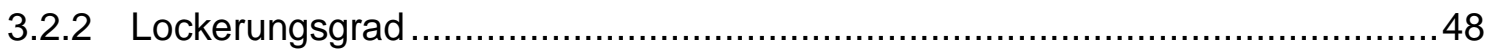

3.3 Modellvermessung - orthodontische Parameter ........................................49

3.3.1 Overjet, Overbite, Little-Index .............................................................. 49

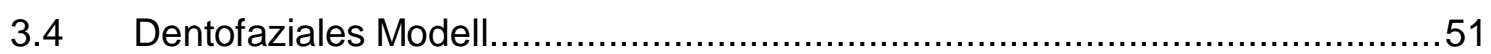

3.4.1 Virtuelles Patientenmodell - Überlagerungspräzision ...................................51

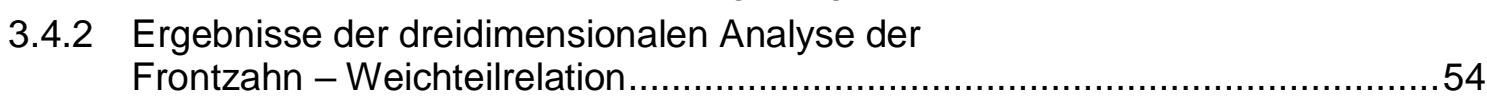

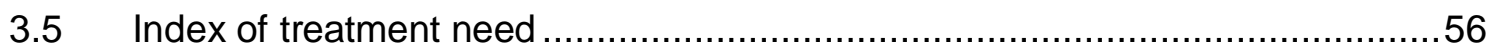

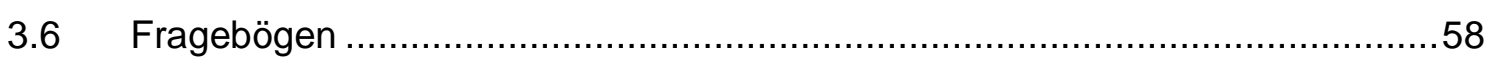

3.6.1 Ergebnisse des patientenindividuellen kieferorthopädischen Fragebogens ......58

3.6.2 Bewusstseinsveränderung hinsichtlich der Mundgesundheit seit der

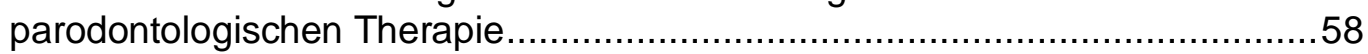

3.6.3 Informationen über eine kieferorthopädische Therapie ................................59

3.6.4 Interesse an einer kieferorthopädischen Behandlung ...................................60

3.6.5 Erwartungen an eine kieferorthopädische Behandlung ..............................61

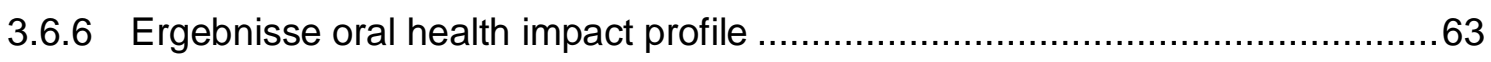

3.6.7 Ergebnisse general / geriatric oral health assessment index ...........................65

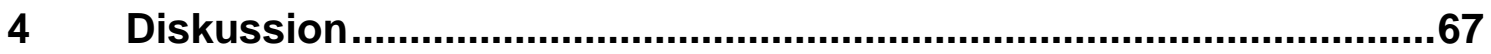

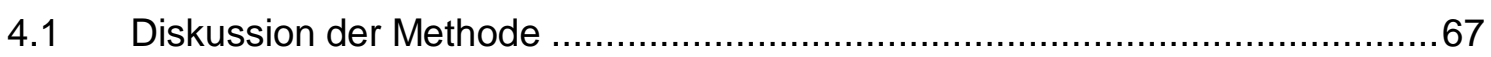

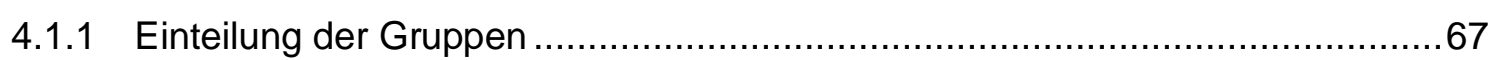

4.1.2 Klinische Datenerhebung und Modellanalyse..............................................68

4.1.3 Digitale Datengenerierung und Erstellung des virtuellen Patienten ..................70

4.2 Diskussion der Ergebnisse ................................................................... 72

4.2.1 Klinisch-parodontale Parameter.............................................................. 72

4.2.2 Orthodontische Parameter ........................................................................ 72

4.2.3 Dreidimensionale Analyse / Virtuelles Patientenmodell ....................................74

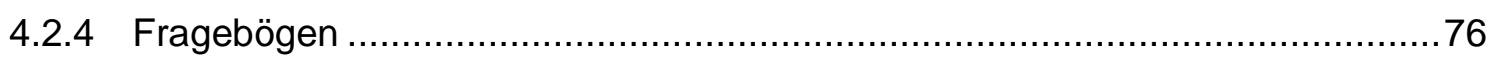

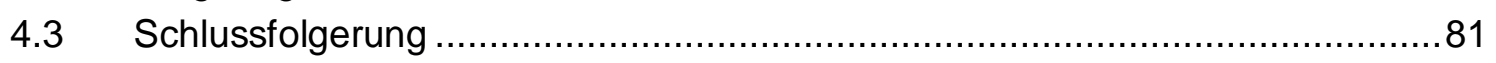

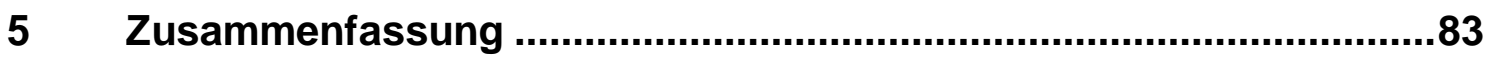

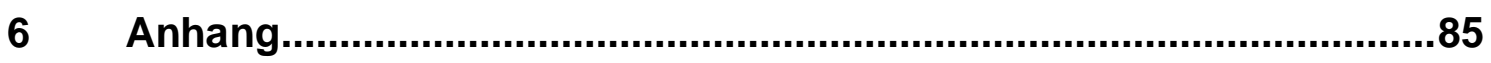

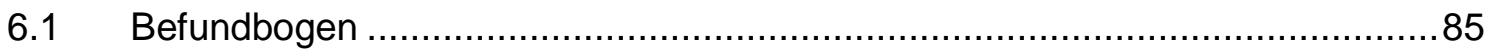

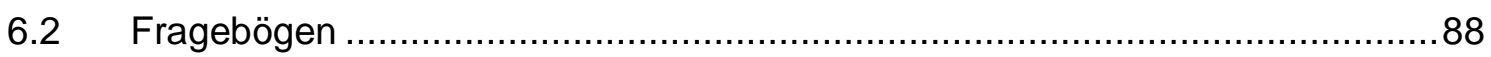

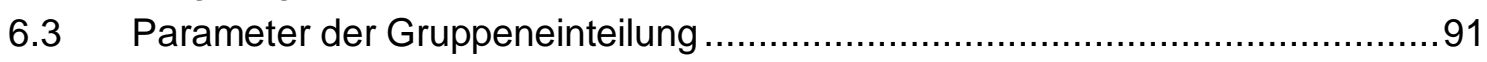

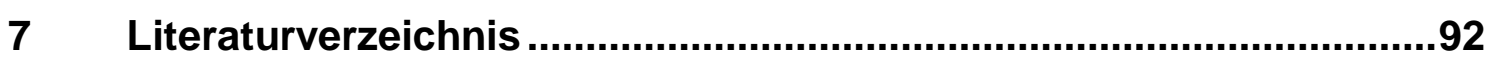




\section{Abbildungsverzeichnis}

Abbildung 1.1: Anatomischer Aufbau des Parodontiums. ........................................ 4

Abbildung 1.2: Pathogeneseschema modifiziert nach Page und Kornman (1997).........7

Abbildung 1.3: Übersicht der neuen Parodontalklassifikation, modifiziert nach Papapanou et al. (2018)

Abbildung 1.4: Übersicht der neuen Parodontalklassifikation - Staging, modifiziert nach Papapanou et al. (2018).

Abbildung 1.5: Übersicht der neuen Parodontalklassifikation - Grading, modifiziert nach Papapanou et al. (2018).

Abbildung 1.6: Frontale und sagittale intraorale Fotoaufnahme eines Patienten mit pathologischen Zahnwanderungen und interdentalen Dunkelzonen.

Abbildung 1.7: Fotostat-Aufnahmen vor und nach der kieferorthopädischparodontologischen Therapie.

Abbildung 1.8: Intraorale Aufnahmen vor, während und nach der

kieferorthopädisch-parodontologischen Therapie.

Abbildung 1.9: FRS und dreidimensionaler Oberflächenscan im Profil vor und nach der kieferorthopädisch-parodontologischen Therapie.

Abbildung 1.10: Dreidimensionale Darstellung eines kieferorthopädischparodontologisch kombiniert therapierten Patienten.

Abbildung 2.1: Dreidimensionale Modelle in Frontansicht und sagittaler Ansicht.........27

Abbildung 2.2: Dreidimensionale Modelle in der Aufsicht des Ober- und Unterkiefers.

Abbildung 2.3: Dreidimensionale Darstellung des overjets, overbites und LittleIndex hier im Unterkiefer.

Abbildung 2.4: Scanner S300 Ortho der Firma Zirkonzahn, Vectra ${ }^{\circledR}$ N5-HeadScanner.

Abbildung 2.5: Registrierung I: Überlagerung der dreidimensionalen Datensätze. ......32

Abbildung 2.6: Registrierung II: Überlagerung der dreidimensionalen Datensätze........32

Abbildung 2.7: Definition der axialen, sagittalen und koronalen Referenzebenen, aufgespanntes Koordinatenkreuz....

Abbildung 2.8: Messungen im dreidimensionalen Raum in dem Programm 3-matic Research 13.0.

Abbildung 2.9: Messungen im dreidimensionalen Raum in dem Programm 3-matic Research 13.0.

Abbildung 3.1: Scatter Plot Registrierung I. Wertepaare der Überlagerung von digitalem Modell und Weichteilscan mit Wangenabhaltern.

Abbildung 3.2: Scatter Plot Registrierung II. Wertepaare der Überlagerung von Weichteilscan in Okklusion und Weichteilscan mit Wangenabhaltern.

Abbildung 3.3: Prävalenz der IOTN-DHC-Level bezogen auf die Patientengruppen „Keine Parodontitis“, „Moderate Parodontitis“, „Schwere Parodontitis“.

Abbildung 3.4: Kreisdiagramm zur Verteilung der Veränderung des Bewusstseins für Zähne, Mund und Mundgesundheit .....

Abbildung 3.5: Kreisdiagramm zur Verteilung des Informationsgewinns über die kieferorthopädischen Behandlungsmöglichkeiten.

Abbildung 3.6: Das Balkendiagramm zeigt das Interesse an einer kieferorthopädischen Behandlung im Allgemeinen.

Abbildung 3.7: Das Balkendiagramm zeigt die Erwartungen an eine kieferorthopädische Behandlung bezogen auf den Erhalt der Zähne 
Abbildung 3.8: Das Balkendiagramm zeigt die Erwartungen an eine kieferorthopädische Behandlung bezogen auf die ästhetische Komponente. ...662

Abbildung 3.9: Die Box-Plots zeigten die OHIP-Summen zwischen den Patientengruppen. 64

Abbildung 3.10: Die Box-Plots zeigen die GOHAI-Summen der Patientengruppen „keine Parodontitis“, „moderate Parodontitis“ und „schwere Parodontitis“. .......66

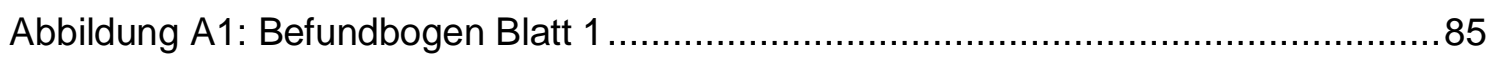

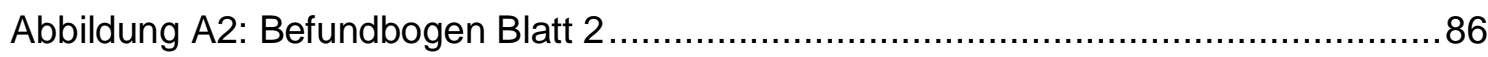

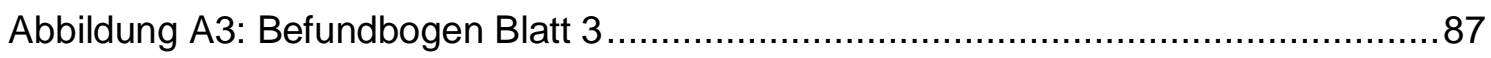

Abbildung A4: Fragebogen zum patientenindividuellen kieferorthopädischen

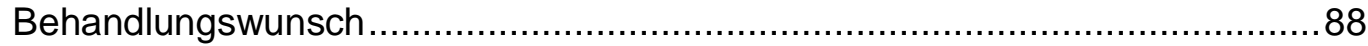

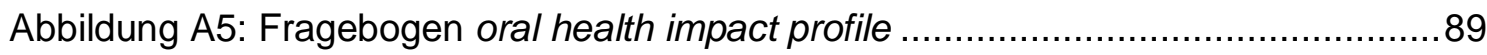

Abbildung A6: Fragebogen general / geriatric oral health assessment index ................90

Abbildung A7: Box Plot Darstellungen zu den Ergebnissen der Parameter der Gruppeneinteilung 


\section{Tabellenverzeichnis}

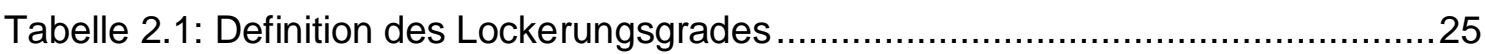

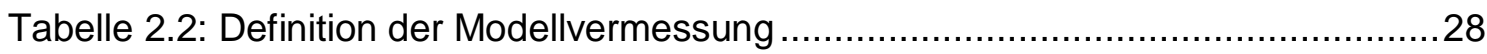

Tabelle 2.3: Definition der anatomischen Messpunkte............................................... 35

Tabelle 2.4: Definition der anatomischen Linien. ..................................................... 36

Tabelle 2.5: Definition der anatomischen Ebenen. ................................................... 36

Tabelle 2.6: Definition des index of treatment need ................................................... 40

Tabelle 3.1: Deskriptive Statistik der Bisslage (Angle-Klassifikation) von allen Patienten bezogen auf die Gruppen....................................................... 45

Tabelle 3.2: Deskriptive Statistik der Anzahl, des Alters und Geschlechts von allen Patienten bezogen auf die Gruppen.

Tabelle 3.3: Vergleich der klinisch-parodontalen Parameter zwischen den Patientengruppen.

Tabelle 3.4: Vergleich des Lockerungsgrades zwischen den Patientengruppen „keine Parodontitis“, „moderate Parodontitis“ und „schwere Parodontitis“. .......48

Tabelle 3.5: Vergleich der Messgenauigkeit der orthodontischen Parameter zu unterschiedlichen Zeitpunkten.

Tabelle 3.6: Vergleich der orthodontischen Parameter $(\mathrm{mm})$ zwischen den Patientengruppen.

Tabelle 3.7: Vergleich der Überlagerungspräzision $(\mathrm{mm})$ zu unterschiedlichen Zeitpunkten.

Tabelle 3.8: Vergleich der dreidimensionalen Parameter der Patientengruppen „keine Parodontitis“, „moderate Parodontitis“ und „schwere Parodontitis“. .......54

Tabelle 3.9: Vergleich der dreidimensionalen Parameter zwischen physiologischem und pathologischem overjet.

Tabelle 3.10: Vergleich der IOTN-DHC Level zwischen den Patientengruppen „keine Parodontitis“, „moderate Parodontitis" und „schwere Parodontitis“.

Tabelle 3.11: Vergleich der OHIP-Summe zwischen den Patientengruppen „keine Parodontitis“, „moderate Parodontitis" und „schwere Parodontitis“.

Tabelle 3.12: Vergleich der GOHAI-Summe zwischen den Patientengruppen „keine Parodontitis“, „moderate Parodontitis“ und „schwere Parodontitis“. 


\section{Abkürzungsverzeichnis}

$\begin{array}{ll}\text { CAL } & \text { clinical attachment loss } \\ \text { CT } & \text { Computertomographie } \\ \text { DHC } & \text { dental health component } \\ \text { DVT } & \text { Digitale Volumentomographie } \\ \text { EL } & \text { esthetic line } \\ \text { FAOP } & \text { functional aesthetic occlusal plane } \\ \text { FRS } & \text { Fernoontgenseitenbild } \\ \text { GOHAI } & \text { general / geriatric oral health assessment index } \\ \text { IOTN } & \text { index of treatment need } \\ \text { LI } & \text { labium inferius } \\ \text { LS } & \text { labium superius } \\ \text { MRT } & \text { Magnetresonanztomographie } \\ \text { NHP } & \text { natural head position } \\ \text { OHIP } & \text { oral health impact profile } \\ \text { RZ } & \text { Rezession } \\ \text { ST } & \text { Sondierungstiefe }\end{array}$




\section{Einleitung}

Parodontitis ist eine, vor allem im adulten Gebiss, weit verbreitete Krankheit, welche in Zukunft immer mehr zunehmen wird und mit starken Einschränkungen der Lebensqualität einhergehen kann (Pihlstrom et al. 2005; Schutzhold et al. 2015; Schiffner 2016). Entzündliche Prozesse multifaktorieller Genese stellen sich klinisch in einer Zerstörung des Zahnhalteapparates dar (Page und Kornman 1997). Pathologische Zahnwanderungen können in bis zu 50 \% der Fälle als Komplikation einer moderaten bis schweren Parodontitis auftreten (Proffit 1978; Towfighi et al. 1997; Brunsvold 2005). Die Herausforderungen bei der Behandlung von Patienten mit starken Zahnmigrationen bestehen darin, in einem stark kompromittierten Zahnhalteapparat einen harmonisch ausgeformten und funktionell stabilen Zahnbogen wiederherzustellen. Alleinige parodontologische oder prothetische Therapieansätze sind häufig nicht ausreichend. Eine kombinierte / interdisziplinäre Therapie, bei der insbesondere der Kieferorthopädie die Aufgabe der Wiederherstellung einer stabilen Zahnposition und Okklusion zuteilwird, ist oftmals angezeigt.

Somit sind orthodontische Behandlungen im Erwachsenenalter seit dem 18. Jahrhundert bekannt, im Rahmen moderner Behandlungskonzepte integriert und nehmen in ihrer Häufigkeit kontinuierlich zu (Goldstein 1953; Thilander 1979; Proffit et al. 1998; Wehrbein et al. 2011; Wong et al. 2018).

Befragungen konnten darlegen, dass Patienten mit einer schweren Parodontitis häufig mit ihrer eigenen dentofazialen Ästhetik unzufrieden sind und über eine verminderte mundgesundheitsbezogene Lebensqualität berichten (Helm et al. 1985; Diedrich 1992; Hirschfeld et al. 2019). Daher untersuchten bisherige Studien die Motivation erwachsener Patienten mit parodontalen Erkrankungen zur kieferorthopädischen Behandlung und konnten einen hohen Motivationsgrad bei geringer Therapieaufklärung darlegen (Ehmer 1981; Bauer und Diedrich 1990; Hirschfeld et al. 2019). Des Weiteren gibt es nach Bernhardt et al. (2019) erste Hinweise auf Zusammenhänge zwischen Malokklusionsgrad und parodontaler Destruktion bei jungen Erwachsenen. Die Korrelationen zwischen dem Schweregrad der Parodontitis und pathologischen Zahnwanderungen im Alter ab 40 Jahren ist jedoch bisher nicht erforscht worden. 
Ziel dieser Studie war es pathologische Zahnwanderungen zu quantifizieren, und zu untersuchen, inwiefern der Grad der Parodontitis mit dem Ausmaß von Zahnmigrationen zusammenhängt. Bezüge zwischen dem Malokklusionsgrad und dem Interesse an einer kieferorthopädischen Behandlung sowie der mundgesundheitsbezogenen Lebensqualität sollten aufgedeckt werden. Darüber hinaus wurde ein virtuelles Patientenmodell erstellt, um zukünftig mögliche Weichteilveränderungen der Lippenregion als Folge der pathologischen Zahnwanderungen zu identifizieren. 


\subsection{Anatomie des Parodontiums}

Unter dem Begriff Parodontium (Zahnhalteapparat) werden vier verschiedene Weich- und Hartgewebe zusammengefasst, welche ein funktionelles System bilden. Hierzu gehören die Gingiva, das Wurzelzement, der Alveolarknochen und das die beiden letzteren Strukturen verbindende Desmodont (Rateitschak et al. 1989). Weski (1921) formulierte ursprünglich den Begriff Paradentium zur Beschreibung der unmittelbaren Umgebung des Zahnes. Diese Formulierung und die Untersuchung von Häupl und Lang (1927) haben die funktionelle Einheit aller vier Gewebsgruppen gezeigt.

Aus klinischer Sicht kann die Gingiva als eine Kombination aus Epithel und Bindegewebe angesehen werden (Schroeder und Listgarten 1997). Sie stellt sowohl einen Teil der Mundschleimhaut als auch den koronalsten Anteil des Parodonts dar. An der mukogingivalen Grenze hat sie ihren Ursprung und umschließt terminal die Zähne mit Hilfe des Saumepithels (Rateitschak et al. 1989). Die Gingiva bildet die erste und wichtigste Grenze gegen Bakterien, welche zu einer Parodontitis führen können (Schroeder und Listgarten 1997). Die Oberflächenbeschaffenheit kann derb, dick und stark gestippelt (dicker Phänotyp) oder zart, dünn und kaum gestippelt sein (dünner Phänotyp) (Eger et al. 1996; Müller et al. 2000).

Das Wurzelzement ist ein avaskuläres mineralisiertes Gewebe, welches die gesamte Wurzeloberfläche bedeckt. Es bildet die Grenzfläche zwischen Wurzeldentin und dem parodontalen Ligament. Seine Funktion ist es, den Zahn in seiner Alveole zu verankern (Cho und Garant 2000).

Der knöcherne Stützapparat wird als Alveolarknochen bezeichnet. Er setzt sich aus den Alveolarfortsätzen des Ober- und Unterkiefers zusammen, welche die zahntragenden Strukturen darstellen. Die Alveolarfortsätze entwickeln sich mit der Zahnbildung und Eruption. Sie sind somit zahnabhängige knöcherne Strukturen (Schroeder 1991). Die Funktion des Alveolarknochens ist es, Kaukräfte zu absorbieren und zu verteilen. Nach Verlust eines Zahns und der damit verbundenen Zerstörung des Parodontiums atrophiert der Knochen durch fehlende funktionelle Beanspruchung (Landsberger 1911).

Das Desmodont, auch parodontales Ligament genannt, ist die verbindende Struktur zwischen dem Zahn und dem Alveolarknochen (Schroeder 1986). Seine 
wichtigste und einzigartige Funktion besteht darin, die Zahnwurzel in der Alveole über Sharpey-Fasern aufzuhängen. Diese Fasern stellen die Verbindung zwischen Alveolarknochen und Wurzelzement dar. Sie sind schräg angeordnet, um Kaukräfte ideal aufnehmen zu können (Feneis 1952; Cho und Garant 2000). Das Desmodont ist kontinuierlich mit dem Bindegewebe der Gingiva verbunden und enthält zahlreiche Blutgefäße und Nerven (Selvig und Mjör 1979).

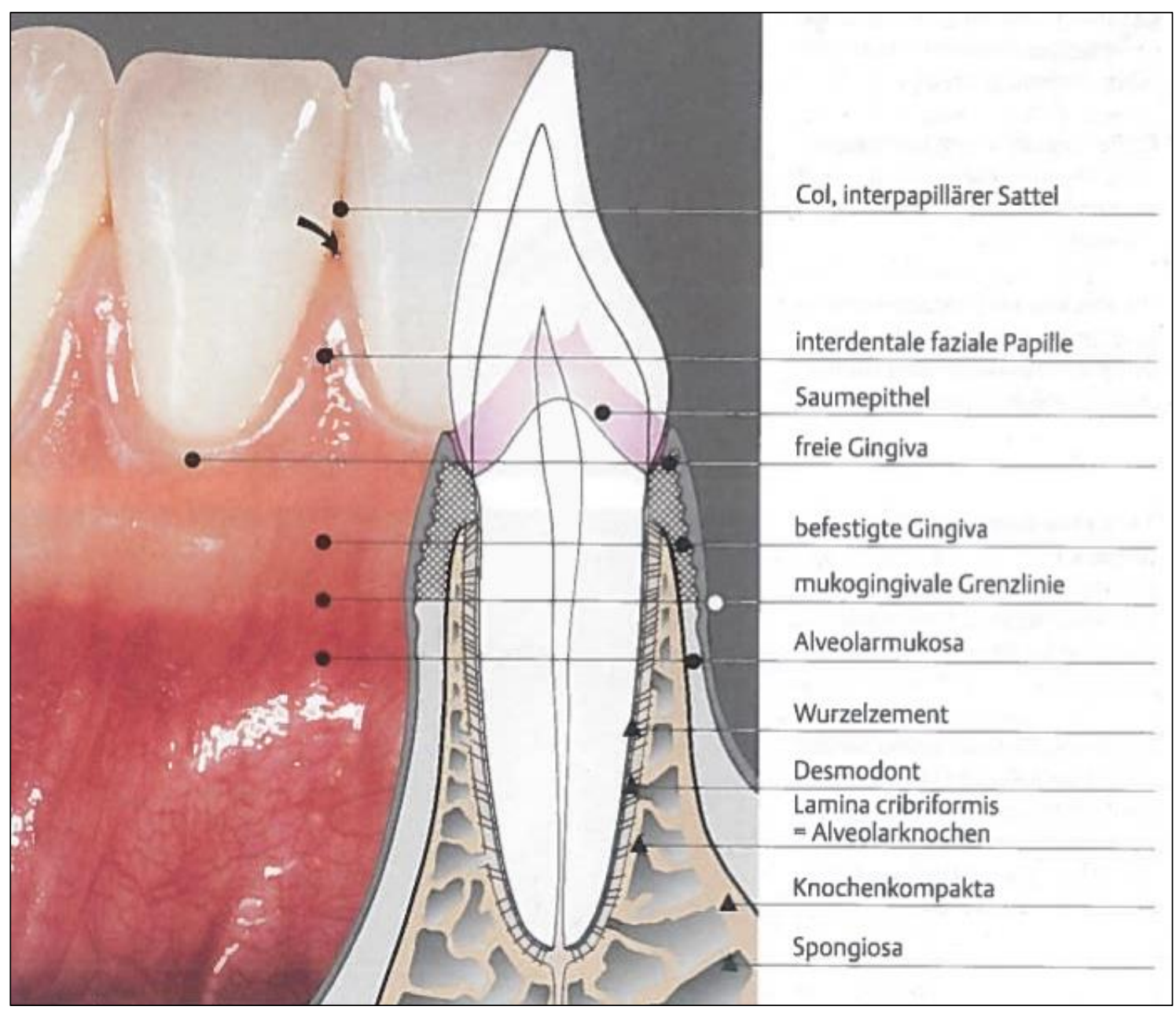

Abbildung 1.1: Anatomischer Aufbau des Parodontiums (Rateitschak et al. 2012). Die Verwendung erfolgt mit freundlicher Genehmigung des Thieme Verlags. 


\subsection{Parodontitis}

Die Parodontitis ist eine multifaktorielle, entzündliche und destruktive Erkrankung des Zahnhalteapparates. Dieser liegt eine Akkumulation von parodontalpathogenen Keimen in Form von supra- und subgingivaler Plaque zugrunde, welche eine Entzündung hervorrufen können (Socransky und Haffajee 2005; Tsuchida et al. 2018). Die dentale Plaque haftet der Zahnoberfläche fest an. Sie setzt sich aus zahlreichen Mikroorganismen zusammen, welche sich in einer extrazellulären Matrix anordnen. Diese wird auch als dentaler Biofilm bezeichnet (Hojo et al. 2009). Die Parodontitis wird durch die verschiedenen Spezies der Bakterien und ihre Eigenschaften, aber auch durch die immunologische Wirtsantwort maßgeblich beeinflusst. Sowohl die den Wirt betreffenden Umwelt- als auch sozioökonomischen Faktoren nehmen Einfluss auf den Verlauf und die Ausprägung der Krankheit (Loe 1983; Newman 1985; Bascones-Martinez und Figuero-Ruiz 2004; Pihlstrom et al. 2005). Betroffene Personen leiden an einer Beeinträchtigung der Lebensqualität durch Zahnverlust, erschwerter Nahrungsaufnahme sowie sozialen Problemen (Popova et al. 2013).

\subsection{1 Ätiologie und Pathogenese der Parodontalerkrankungen}

Der zentrale ätiologische Faktor bei der Parodontitisentstehung ist die Etablierung einer parodontalpathogenen Mikroflora. Die Akkumulation von bakterieller Plaque auf der Zahnoberfläche ruft eine reproduzierbare Entzündungsantwort im gingivalen Gewebe hervor. Die Plaqueentfernung führt zum Rückgang der klinischen Entzündungszeichen (Loe et al. 1965; Theilade et al. 1966). Ausgereifte vollpathogene Plaque etabliert sich nach 7-14 Tagen und ist umso parodontalpathogener, je mehr gramnegative und anaerobe Erreger in ihrer Mikroflora enthalten sind (Newman 1985; Rateitschak et al. 1989). Zunächst entsteht als Reaktion auf die Plaqueanlagerung in Form eines Biofilms auf der Zahnoberfläche eine initiale Gingivitis (Loe et al. 1965). Aus einer unbehandelten etablierten Gingivitis, welche nach 3-4 Wochen Plaqueakkumulation entsteht und bei der erste Abbauprozesse im bindegewebigen Zahnhalteapparat stattfinden, kann eine destruktive Parodontitis entstehen. Diese geht mit einer apikalen Proliferation des Taschenepithels einher. Hierdurch kommt es zu einer echten Taschenbildung. Außerdem findet ein fortschreitender bindgewebiger Attachmentverlust sowie 
Resorption des Alveolarknochens statt (Schroeder 1997). Subgingivale Plaqueakkumulation und Bildung von Gingivaexsudat begünstigen die Besiedlung mit parodontalpathogenen Keimen (Theilade 1986). Diese unterscheiden sich in ihrer Pathogenität und nehmen unterschiedlichen Einfluss auf die Progression der Parodontitis (Slots 2004; Socransky und Haffajee 2005). $\mathrm{Ab}$ einer bestimmten Bakteriendichte im Biofilm steigt die Konzentration von bakteriellen Signalmolekülen an, woraus das sog. Quorum sensing resultieren kann. Dabei handelt es sich um eine Synchronisierung der Bakterien und gemeinsame vorteilhafte Genexpression. Bakterien können durch das Quorum Sensing Prozesse wie die Änderung des Pathogenitätsfaktors begünstigen (Rutherford und Bassler 2012).

Eine etablierte Gingivitis kann über Jahre stagnieren und ohne Progression bestehen. Entscheidend und bestimmend für das Auftreten und Ausmaß der parodontalen Destruktion ist die Wirtsantwort auf die Herausforderung durch die Plaque. Sobald bei den Wirtsfaktoren Störungen oder Schwächen vorliegen, kann eine bislang nichtpathogene Plaque parodontalpathogen werden. Ebenso kann eine bis dahin erfolgreiche Wirtsabwehr nicht mehr ausreichen, wenn die Zusammensetzung der Plaque Modifikationen mit Verschiebung zu erhöhter Pathogenität erfährt. Die Wirtsabwehr wird aus mechanischen Barrieren, wie epithelialen Strukturen, chemischen Barrieren, wie dem Sulkusfluid, aber auch durch das Immunsystem zusammengesetzt (Meyle und Chapple 2015). Entscheidend ist die Einflussnahme auf Bindegewebe und Knochenstoffwechsel, welche sich durch deren Abbau klinisch zeigt (Page und Kornman 1997). Die Kernelemente des klassischen Modells der Krankheitspathogenese von Page und Kornman (1997), welche die dynamischen Wechselwirkungen zwischen verschiedenen mikrobiellen Faktoren und Wirtsfaktoren darstellt, zeigt Abbildung 1.2. Resultat der entzündlichen Erkrankung ist der irreversible Verlust von parodontalem Gewebe. Dies lässt sich durch erhöhte bzw. pathologische Sondierungstiefen klinisch messen. Die parodontale Destruktion führt bei stetiger Progression und ohne adäquate Behandlung zur Lockerung der Zähne und letztendlich zum Zahnverlust. Zudem können unkontrollierte Zahnwanderungen auftreten (Pihlstrom et al. 2005). 


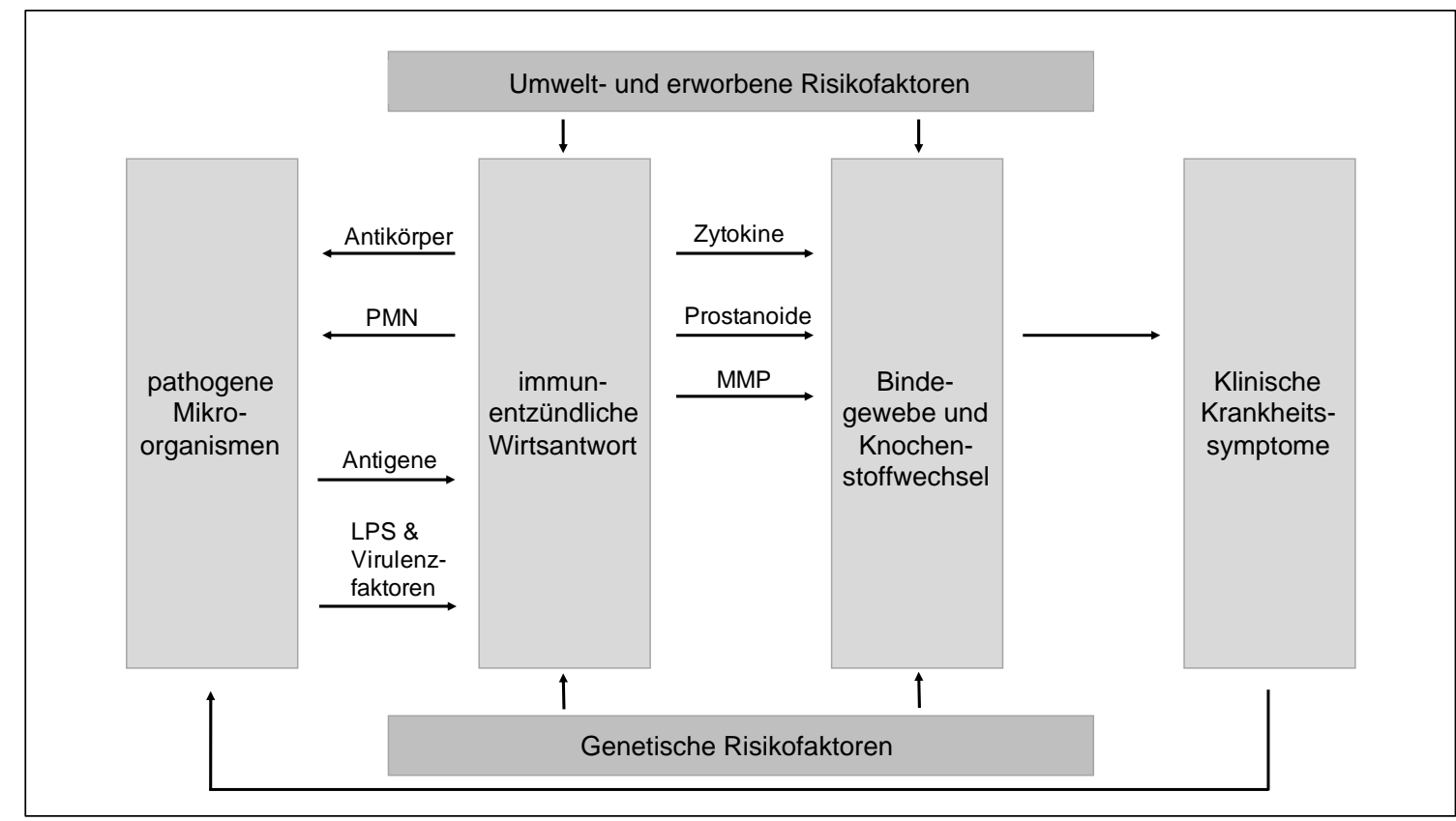

Abbildung 1.2: Pathogeneseschema modifiziert nach Page und Kornman (1997). Die Verwendung erfolgt mit freundlicher Genehmigung des John Wiley and Sons Verlags.

\subsubsection{Risikofaktoren}

Für die Entstehung einer Parodontitis sind verschiedene Risikofaktoren bekannt, die sich in primäre und sekundäre, sowie verhaltensbedingte Faktoren gliedern lassen.

Als primärer Risikofaktor ist die Zusammensetzung des Biofilms beschrieben, der aus Bakterien, extrazellulärer Matrix und Proteinen besteht. Bisher wurden über 700 Mikroorganismen der Mundhöhle erforscht, die in ihrer Pathogenität variieren (Hojo et al. 2009; Dewhirst et al. 2010; Verma et al. 2018).

Beispielhaft seien als besonders parodontalpathogene Bakterien Aggregatibacter actinomycetemcomitans und Porphyromonas gingivalis genannt (Slots 2004). Socransky et al. (1998) haben die Organisation und die Interaktion der verschiedenen Bakterien in Komplexen dargestellt.

Sekundäre Risikofaktoren unterteilen sich sowohl in veränderbare als auch in nicht veränderbare Faktoren, welche Einfluss auf den Krankheitsverlauf nehmen. Zu den nicht veränderbaren gehört ein erhöhtes Lebensalter, das für jede Form der chronischen Erkrankung als Risikofaktor angenommen wird (Eke et al. 2012; Reynolds 2014). Ein erhöhtes Lebensalter ist der am stärksten assoziierte Faktor bezüglich des Attachmentverlusts mit einer Odds Ratio für 35 bis 44-jährige von 1,72 und einer Odds Ratio von 9,01 für 65 bis 74-jährige (Grossi et al. 1994). 
Eine Odds Ratio beschreibt wie viel größer die Wahrscheinlichkeit für das Auftreten einer Erkrankung in einer Gruppe mit Risikofaktor ist, verglichen mit einer Gruppe ohne diesen Risikofaktor.

Verhaltensbedingte und veränderbare Risikofaktoren beziehen sich auf den Verzehr von Genussmitteln, die individuelle Lebensweise und die hieraus folgenden Erkrankungen. Der Konsum von Tabak erhöht das Risiko, an einer Parodontitis zu erkranken im Vergleich zu Nichtrauchern ähnlichen Alters und sozioökonomischen Status (Preber und Bergstrom 1986; Bergstrom 1989). Die Wahrscheinlichkeit an einer Parodontitis zu erkranken ist für starke Raucher um den Faktor fünf erhöht (Stoltenberg et al. 1993; Grossi et al. 1994; Tonetti 1998). Bei Rauchern sind die Abwehrkräfte des Parodontalgewebes durch Reduktion der Immunantwort auf Parodontalpathogene und eine verminderte Wundheilungsfähigkeit beeinträchtigt (Preber und Bergstrom 1990; Palmer et al. 2005). Es kommt zu einer vermehrten Freisetzung proinflammatorischer Zytokine und verminderter Produktion von Antikörpern gegen gramnegative Bakterien (Van Dyke und Sheilesh 2005), sodass es bei Rauchern im Vergleich zu Nichtrauchern zu vermehrten Zahnverlusten und einer stärkeren Knochenatrophie kommt (Bergstrom 1989; Grossi et al. 1994).

Patienten, die unter Diabetes mellitus Typ 1 und Typ 2 leiden, haben ein höheres Risiko an einer Parodontitis zu erkranken, und die parodontale Destruktion nimmt bei schlecht eingestellten Blutzuckerwerten einen schwereren Verlauf (Thorstensson und Hugoson 1993; Clarke und Hirsch 1995; Soskolne und Klinger 2001). Aufgrund des chronisch hohen Blutzuckerspiegels kommt es im Blut zur Glykierung von Proteinen und zur Bildung sog. advanced glycation endproducts. Diese können über die Bindung an einen entsprechenden Rezeptor die Aktivierung der Produktion von proinflammatorischen Zytokinen induzieren. Außerdem werden Osteoklasten, Kollagenasen und Matrixmetalloproteinasen aktiviert. Die Destruktion des Alveolarknochens und Bindegewebes sind die Folgen (Soskolne und Klinger 2001). Es besteht ein bidirektionaler Zusammenhang zwischen einer parodontalen Erkrankung und einem Diabetes mellitus. Die Entzündungsreaktion der Parodontitis verstärkt die Insulinresistenz der Zellen und eine adäquate Blutzuckereinstellung wird erschwert. Außerdem korreliert ein andauernd schlechter Blutzuckerspiegel mit dem Schweregrad der Parodontitis (Taylor 2001). Schlecht eingestellte Diabetiker weisen häufiger 
erhöhte Sondierungstiefen und einen verstärkten Alveolarknochenverlust auf als gesunde Patienten (Thorstensson und Hugoson 1993).

Ebenso ist bewiesen, dass psychologische Faktoren in Form von Stress den Schweregrad der Parodontitis beeinflussen. Es wird angenommen, dass Stress aufgrund seiner immunsuppressiven Wirkung zu einer Verschlechterung des parodontalen Zustandes führt (Doyle und Bartold 2012).

\subsubsection{Epidemiologie}

Parodontitis gilt als eine weltweit stark verbreitete Erkrankung in der modernen Gesellschaft. Im Jahr 2016 war Parodontitis die elfthäufigste Erkrankung in der gesamten Weltbevölkerung mit einer Zunahme um 2,3\% in den letzten zehn Jahren (Vos et al. 2017). Bezogen auf die gesamte Weltbevölkerung betrug die Prävalenz für eine schwere Parodontitis im Jahr 2010 10,8 \%, das entsprach 743 Millionen Menschen (Papapanou und Susin 2017). Im Jahr 2017 betrug die Erkrankungsrate 10-15 \% (Gross et al. 2017).

In Deutschland zeigen epidemiologische Daten in Bezug auf die Mundgesundheit, dass sowohl Zahnkaries als auch Parodontalerkrankungen weit verbreitet sind (Jordan et al. 2014). Die verbreitetsten und wichtigsten Ursachen für Zahnverluste sind Karies und Parodontitis (Pihlstrom et al. 2005; Rozier et al. 2017). Das Risiko, an einer moderaten oder schweren Form der Parodontitis zu erkranken, nimmt mit fortschreitendem Alter zu (Papapanou und Susin 2017). Im Rahmen der fünften Deutschen Mundgesundheitsstudie von 2014 wurde ein Überblick über die Entwicklung parodontaler Erkrankungen in der deutschen Bevölkerung erstellt. Die Zahl der an Parodontitis erkrankten Menschen nahm im Vergleich zur vierten Deutschen Mundgesundheitsstudie zwar ab, aufgrund des demographischen Wandels wird aber in Zukunft ein höherer Behandlungsbedarf prognostiziert (Schiffner 2016). Der Behandlungsbedarf wird weiter zunehmen, weil bei älteren Menschen und Senioren immer mehr natürliche Zähne in situ verbleiben (Schutzhold et al. 2015). Jeder zweite jüngere Erwachsene im Alter von 35 - 44 Jahren ist von einer parodontalen Erkrankung betroffen. Hiervon leidet jeder zehnte an einer schweren Form der Parodontitis. In der Altersklasse zwischen 65 - 74 Jahren hat ebenfalls jeder zweite eine Parodontitis. Jeder fünfte weist eine schwere Ausprägung der Erkrankung auf (Schiffner 2016). 
Aufgabe der zahnärztlichen Gemeinschaft ist es, bereits moderate parodontale Erkrankungen zu erkennen und zu behandeln (Schutzhold et al. 2015). Durch ein besseres Verständnis für Folgen und Ursachen der Erkrankung können auch Hausärzte zu einer Verbesserung der Allgemeingesundheit beitragen (Gross et al. 2017).

\subsubsection{Definition und Klassifikation der Parodontalerkrankungen}

Parodontale Erkrankungen werden seit Juni 2018 neu eingeteilt. Zuvor bestand die Einteilung in chronische und aggressive Parodontitis aus dem Jahr 1999, welche durch ein staging und grading ersetzt wurde (Armitage 1999). Die Ergebnisse des "World Workshop on the Classification of Periodontal and Periimplant Diseases and Conditions" basieren auf vier Konsensusberichten sowie auf dem Klassifikationsschema von 2017 (Berglundh et al. 2018; Caton et al. 2018; Chapple et al. 2018; Jepsen et al. 2018; Papapanou et al. 2018). Nach der Pathophysiologie und dem klinischen Krankheitsbild werden drei Formen der parodontalen Erkrankung unterschieden.

Diejenigen Formen, welche in die Kategorie chronische und aggressive Erkrankung eingeteilt wurden, werden nun in der Kategorie Parodontitis zusammengefasst. Des Weiteren wird zwischen nekrotisierender Parodontitis und Parodontitis als Manifestation systemischer Erkrankungen differenziert. Der Klassifikation liegt eine staging und grading Matrix zugrunde (Abbildung 1.3, Abbildung 1.4, Abbildung 1.5). Das staging beschreibt das Stadium der parodontalen Erkrankung und umfasst vier Stufen. Es ist weitestgehend vom Schweregrad der Erkrankung abhängig, welcher anhand klinischer und röntgenologischer Befunde diagnostiziert wird. Außerdem hat die Komplexität Einfluss auf das Krankheitsmanagement. Das grading gibt ergänzende Informationen hinsichtlich Verlaufsform der Parodontitis in der Vergangenheit und individuellen Risikofaktoren, sowie systemischen Erkrankungen. Dabei wird in ein niedriges, mittleres und hohes Risiko für die Krankheitsprogression eingeteilt. (Papapanou et al. 2018; Tonetti et al. 2018). 
Parodontale Erkrankungen und Zustände

\begin{tabular}{|c|c|c|c|c|c|c|c|c|c|c|}
\hline \multicolumn{3}{|c|}{$\begin{array}{c}\text { Parodontale } \\
\text { Gesundheit, } \\
\text { Gingivale Erkrankungen }\end{array}$} & \multicolumn{3}{|c|}{ Parodontitis } & \multicolumn{5}{|c|}{$\begin{array}{c}\text { Andere das Parodont betreffende } \\
\text { Zustände }\end{array}$} \\
\hline $\begin{array}{l}\text { Paro- } \\
\text { dontale } \\
\text { und } \\
\text { gingivale } \\
\text { Gesund- } \\
\text { heit }\end{array}$ & $\begin{array}{c}\text { Gingivitis: } \\
\text { Biofilm } \\
\text { induziert }\end{array}$ & $\begin{array}{l}\text { Gingivale } \\
\text { Erkran- } \\
\text { kungen: } \\
\text { Nicht } \\
\text { Biofilm- } \\
\text { induziert }\end{array}$ & $\begin{array}{l}\text { Nekroti- } \\
\text { sierende } \\
\text { paro- } \\
\text { dontale } \\
\text { Erkran- } \\
\text { kungen }\end{array}$ & $\begin{array}{l}\text { Paro- } \\
\text { dontitis }\end{array}$ & $\begin{array}{c}\text { Paro- } \\
\text { dontitis als } \\
\text { Mani- } \\
\text { festation } \\
\text { von } \\
\text { System- } \\
\text { erkran- } \\
\text { kungen } \\
\end{array}$ & $\begin{array}{l}\text { System- } \\
\text { ische } \\
\text { Erkran- } \\
\text { kungen } \\
\text { mit } \\
\text { Einfluss } \\
\text { auf das } \\
\text { Parodont } \\
\end{array}$ & $\begin{array}{c}\text { Paro- } \\
\text { dontale } \\
\text { Abs- } \\
\text { zesse / } \\
\text { Endo- } \\
\text { Paro- } \\
\text { Läsion }\end{array}$ & $\begin{array}{l}\text { Muko- } \\
\text { gingivale } \\
\text { Defor- } \\
\text { mitäten } \\
\text { und } \\
\text { Zustände }\end{array}$ & $\begin{array}{l}\text { Traum- } \\
\text { atische } \\
\text { okklusale } \\
\text { Kräfte }\end{array}$ & $\begin{array}{l}\text { Zahn- und } \\
\text { Zahn- } \\
\text { ersatz } \\
\text { asso- } \\
\text { ziierte } \\
\text { Faktoren }\end{array}$ \\
\hline
\end{tabular}

Abbildung 1.3: Übersicht der neuen Parodontalklassifikation, modifiziert nach Papapanou et al. (2018). Die Verwendung erfolgt mit freundlicher Genehmigung des John Wiley and Sons Verlags.

\begin{tabular}{|c|c|c|c|c|c|}
\hline \multicolumn{6}{|c|}{ Parodontitis: Staging } \\
\hline \multicolumn{2}{|c|}{ Parodontitis-Stadien } & Stadium I & Stadium II & Stadium III & Stadium IV \\
\hline \multirow{3}{*}{ Schwere } & $\begin{array}{l}\text { Höchster Interdentaler } \\
\text { CAL }\end{array}$ & $1-2 \mathrm{~mm}$ & 3-4 mm & $\geq 5 \mathrm{~mm}$ & $\geq 5 \mathrm{~mm}$ \\
\hline & $\begin{array}{l}\text { Röntgenologischer } \\
\text { Knochenabbau }\end{array}$ & $\begin{array}{l}\text { koronales Drittel } \\
\quad(<15 \%)\end{array}$ & $\begin{array}{l}\text { koronales Drittel } \\
\quad(15-33 \%)\end{array}$ & $\begin{array}{l}\text { Mittleres oder } \\
\text { apikales } \\
\text { Wurzeldrittel }\end{array}$ & $\begin{array}{l}\text { Mittleres oder } \\
\text { apikales } \\
\text { Wurzeldrittel }\end{array}$ \\
\hline & Zahnverlust & \multicolumn{2}{|c|}{ Kein Zahnverlust aufgrund von Parodontitis } & $\begin{array}{l}\text { Zahnverlust } \\
\text { aufgrund von } \\
\text { Parodontitis von } \\
\leq 4 \text { Zähnen }\end{array}$ & $\begin{array}{l}\text { Zahnverlust } \\
\text { aufgrund von } \\
\text { Parodontitis von } \\
\leq 5 \text { Zähnen }\end{array}$ \\
\hline Komplexität & lokal & $\begin{array}{c}\text { maximale } \\
\text { Sondierungstiefe } 3-4 \mathrm{~mm} \text {, } \\
\text { hauptsächlich } \\
\text { horizontaler } \\
\text { Knochenabbau }\end{array}$ & $\begin{array}{c}\text { maximale } \\
\text { Sondierungstiefe 4-5 mm, } \\
\text { hauptsächlich } \\
\text { horizontaler } \\
\text { Knochenabbau }\end{array}$ & $\begin{array}{l}\text { Zusätzlich } \\
\text { zu Stadium-II: } \\
\text { Sondierungstiefe } \\
\geq 6 \text { mm, } \\
\text { vertikaler } \\
\text { Knochenabbau } \\
\geq 3 \mathrm{~mm}, \\
\text { Furkations- } \\
\text { beteiligung Grad } \\
\text { II / III, moderater } \\
\text { Kammdefekt }\end{array}$ & $\begin{array}{c}\text { Zusätzlich zu } \\
\text { Stadium-III: } \\
\text { Komplexe } \\
\text { Rehabilitation } \\
\text { notwenig, } \\
\text { mastikatorische } \\
\text { Dysfunktion, } \\
\text { sekundäre } \\
\text { okklusale } \\
\text { Trauma, } \\
\text { Zahnbeweg- } \\
\text { lichkeit } \geq \text { Grad 2, } \\
\text { Zahnwanderung, } \\
\text { Auffächerung, } \\
\text { weniger als 20 } \\
\text { Zähne, schwerer } \\
\text { Kammdefekt } \\
\end{array}$ \\
\hline $\begin{array}{l}\text { Ausmaß und } \\
\text { Verteilung }\end{array}$ & $\begin{array}{l}\text { Als Deskriptor zum } \\
\text { Stadium hinzufügen }\end{array}$ & \multicolumn{4}{|c|}{$\begin{array}{c}\text { Für jedes Stadium Ausmaß als lokalisiert, über } 30 \% \text { der Zähne betroffen, generalisiert oder } \\
\text { Molaren-Inzisiven-Muster beschrieben }\end{array}$} \\
\hline
\end{tabular}

Abbildung 1.4: Übersicht der neuen Parodontalklassifikation - Staging, modifiziert nach Papapanou et al. (2018). Die Verwendung erfolgt mit freundlicher Genehmigung des John Wiley and Sons Verlags. 


\begin{tabular}{|c|c|c|c|c|c|}
\hline \multicolumn{6}{|c|}{ Parodontitis: Grading } \\
\hline $\begin{array}{c}\text { Parodontitis- } \\
\text { Grade }\end{array}$ & & & $\begin{array}{c}\text { Grad A } \\
\text { Langsame } \\
\text { Progress- } \\
\text { ionsrate }\end{array}$ & $\begin{array}{c}\text { Grad B } \\
\text { Moderate } \\
\text { Progress- } \\
\text { ionsrate }\end{array}$ & $\begin{array}{c}\text { Grad C } \\
\text { Schnelle } \\
\text { Progress- } \\
\text { ionsrate }\end{array}$ \\
\hline \multirow{3}{*}{ Primäre Kriterien } & $\begin{array}{l}\text { Direkte Evidenz für } \\
\text { Progression }\end{array}$ & $\begin{array}{l}\text { longitudinale Daten } \\
\text { (röntgenologisch oder } \\
\text { Verlust an CAL) }\end{array}$ & $\begin{array}{l}\text { Evidenz für keinen } \\
\text { Abbau über } 5 \text { Jahre }\end{array}$ & $\begin{array}{c}<\text { als } 2 \mathrm{~mm} \text { über } 5 \\
\text { Jahre }\end{array}$ & $\begin{array}{c}\geq 2 \mathrm{~mm} \text { über } 5 \\
\text { Jahre }\end{array}$ \\
\hline & \multirow[b]{2}{*}{$\begin{array}{l}\text { indirekte Evidenz für } \\
\text { Progression }\end{array}$} & Knochenabbau / Alter & $<0,25$ & $0,25-1,0$ & $>1,0$ \\
\hline & & Fall-Phänotyp & $\begin{array}{l}\text { Biofilmauf- } \\
\text { lagerungen mit } \\
\text { wenig } \\
\text { parodontalem } \\
\text { Abbau }\end{array}$ & $\begin{array}{c}\text { parodontale } \\
\text { Destruktion } \\
\text { entspricht } \\
\text { Biofilmauf- } \\
\text { lagerungen }\end{array}$ & $\begin{array}{l}\text { Mehr parodontale } \\
\text { Destruktion als } \\
\text { Biofilmauff- } \\
\text { lagerungen, } \\
\text { schnelle oder } \\
\text { periodische } \\
\text { Progression, kein } \\
\text { Ansprechen auf } \\
\text { Therapie } \\
\end{array}$ \\
\hline \multirow[b]{2}{*}{ Grad-Modifikatoren } & \multirow[b]{2}{*}{ Risikofaktoren } & Rauchen & Nichtraucher & $\begin{array}{c}<10 \text { Zigaretten / } \\
\text { Tag }\end{array}$ & $\geq 10$ Zigaretten / \\
\hline & & Diabetes & $\begin{array}{l}\text { Normoglykämisch } \\
\text { ohne } \\
\text { diagnostizierten } \\
\text { Diabetes }\end{array}$ & $\begin{array}{l}\text { HbA1c }<7,0 \text { bei } \\
\text { Diabetes- } \\
\text { erkrankung }\end{array}$ & $\begin{array}{l}\text { HbA1c } \geq 7,0 \text { bei } \\
\text { Diabetes- } \\
\text { erkrankung }\end{array}$ \\
\hline
\end{tabular}

Abbildung 1.5: Übersicht der neuen Parodontalklassifikation - Grading, modifiziert nach Papapanou et al. (2018). Die Verwendung erfolgt mit freundlicher Genehmigung des John Wiley and Sons Verlags.

\subsection{Zusammenhang zwischen Parodontitis und pathologischen Zahnstellungsänderungen}

Als Folge einer moderaten bis schweren Parodontitis können pathologische Zahnwanderungen auftreten. Die Prävalenz, pathologischer Zahnwanderungen bei parodontal Erkrankten liegt bei bis zu 50 \% (Brunsvold 2005). Sie sind eine häufige Motivation für Betroffene, eine parodontale Therapie in Betracht zu ziehen. Die Ätiologie der Zahnmigration scheint multifaktoriellen Ursprungs zu sein. Die parodontale Destruktion spielt eine wesentliche Rolle in der Entstehung pathologischer Migrationen, begleitet von Zahnverlusten und akuten gingivalen Entzündungen (Towfighi et al. 1997; Brunsvold 2005). Anhand der Odds Ratio lässt sich zeigen, dass pathologische Zahnwanderungen mit zunehmender parodontaler Destruktion verstärkt auftreten. Die Zunahme der Zahnwanderungen bei einer schweren im Vergleich zu einer einer moderaten Parodontitis beträgt gemessen an der Odds Ratio 5,02 (Martinez-Canut et al. 1997). 
Klinisch lassen sich verschiedene morphologische Ausprägungen beobachten. Es kommt zur Auffächerung und Labialkippung der Oberkieferfrontzähne, sowie zur Diastemabildung, Rotation, Extrusion und Kippung (Eliasson et al. 1982; Towfighi et al. 1997; Oh 2011). Außerdem zeigt sich, dass durch Verlust der Dentition in den Stützzonen aufgrund parodontaler Destruktion eine Absenkung der Bisslage auftreten kann, wodurch die Auffächerung beziehungsweise Protrusion der Oberkieferfrontzähne begünstigt wird (Towfighi et al. 1997; Shifman et al. 1998; Brunsvold 2005).

Insbesondere nach starker parodontaler Destruktion können Weichteilkräfte durch Zunge, Lippen und Wangen zu Zahnbewegungen führen (Proffit 1978; Chasens 1979). Diese werden bei nicht erkrankten Patienten normalerweise durch das gesunde Parodont ausgeglichen und es liegt ein Gleichgewicht aller Kräfte vor. Ein gestörtes Gleichgewicht begünstigt die Migration der Frontzähne (Brunsvold 2005). Durch entzündliche Prozesse in der parodontalen Tasche kann es in Folge von Druckveränderungen zum pathologischen Abdriften von Zähnen kommen (Towfighi et al. 1997).

\subsubsection{Kombinierte kieferorthopädisch-parodontale Therapie}

Die Herausforderungen, welche Patienten mit pathologischen Zahnwanderungen und starker parodontaler Destruktion mit sich bringen, sind häufig nicht durch eine alleinige Parodontaltherapie zu lösen. Aufgrund der Zahnlockerung und Wanderung der Zähne kommt es zu einer progredienten Instabilität der betroffenen Zähne durch unphysiologische Zahnvorkontakte. Zusätzlich erfolgt bei jeder Kaubelastung der Zähne mit zunehmender parodontaler Destruktion eine weitere progrediente Auslenkung der Zähne als sekundäres okklusales Trauma. Somit entsteht ein Circulus vitiosus an dessen Ende häufig dann der Zahnverlust steht. Daher kann zur Wiederherstellung einer stabilen Zahnposition eine orthodontische Bewegung der Zähne in ihre alveoläre Hülle indiziert sein (siehe 1.3.2).

Mit dem funktionellen Mehrwert einer kombiniert kieferorthopädischparodontalen Therapie geht zusätzlich der für den Patienten bedeutende ästhetische Mehrwert einher. Die initialen Schwellungen des Weichgewebes nach erfolgreicher Parodontaltherapie klingen ab und es entstehen in zervikalen Bereichen häufig interdentale Dunkelräume (schwarze Dreiecke). Somit ist die 
dentofaziale Ästhetik dieser Patienten stark eingeschränkt (Tugnait und Clerehugh 2001; Sharma und Park 2010). Pathologische Zahnwanderungen als Resultat starker parodontaler Destruktion tragen ebenso zu einer verminderten Ästhetik bei (Brunsvold 2005). Diese Veränderungen können das Selbstbewusstsein und die Lebensqualität der Patienten drastisch reduzieren (Klages et al. 2004; Sun et al. 2017).

Die orthodontische Behandlung hat zum Ziel Zahnfehlstellungen zu korrigieren. In den letzten Jahrzehnten ist die Anzahl der kieferorthopädischen Therapien bei Erwachsenen kontinuierlich angestiegen (Thilander 1979; Proffit et al. 1998; Wong et al. 2018). Dabei spielt die kieferorthopädische Therapie im Erwachsenenalter bei parodontal kompromittierten Patienten eine immer größer werdende Rolle. Sie dient der vollständigen Rehabilitation hinsichtlich Ästhetik und Funktion mit einer guten Langzeitprognose (Ong und Wang 2002).

Pathologische Zahnmigrationen, die in tiefen Bissen, okklusalen Traumata sowie aufgefächerten Frontzähnen mit Labialkippung resultieren können und mit der Schwere der Parodontitis zunehmen, sind nach erfolgreicher Parodontaltherapie durch kieferorthopädische Behandlungen therapierbar. Zähne, für die als alleinige Option zuvor die Extraktion in Betracht gezogen wurde, können so erhalten werden. Es kann ein guter Parodontalstatus erreicht werden, wenn die orthodontische Therapie sorgfältig geplant wird (Cirelli et al. 2006; Xie et al. 2014; Jepsen et al. 2015). Durch eine Optimierung der Zahnstellung werden adäquate Mundhygiene und Pflegeoptionen für den Patienten vereinfacht. Dies begünstigt die Aufrechterhaltung eines entzündungsfreien stabilen Parodonts mit reduzierter Gefahr eines Rezidivs (Gkantidis et al. 2010). Vorangegangene Studien konnten zeigen, dass die kieferorthopädische Therapie parodontale Untersuchungsparameter, wie die Sondierungstiefe, das Bluten auf Sondieren und die Plaquemenge nach erfolgreicher Parodontaltherapie positiv beeinflussen kann. Voraussetzung ist eine strenge Biofilmkontrolle während der aktiven orthodontischen Therapie (Carvalho et al. 2018).

Eine bessere Ästhetik während des Lächelns und ein gesteigertes Selbstvertrauen, respektive eine mundgesundheitsbezogene Lebensqualität, sind ebenfalls durch die interdisziplinäre orthodontisch-parodontologische Therapie zu erwarten und werden von den Patienten als besonders wichtiger 
Faktor hinsichtlich der Therapiewahl beschrieben (Klages et al. 2004; Pabari et al. 2011; Xie et al. 2014). Entscheidend für den Erfolg einer kombinierten Therapie, ist ein stabiler und entzündungsfreier Mundhygienestatus, sowie Parodontalzustand. Dies gelingt nur durch ein hohes $\mathrm{Maß}$ an Compliance seitens des Patienten (Ong und Wang 2002; Gkantidis et al. 2010).
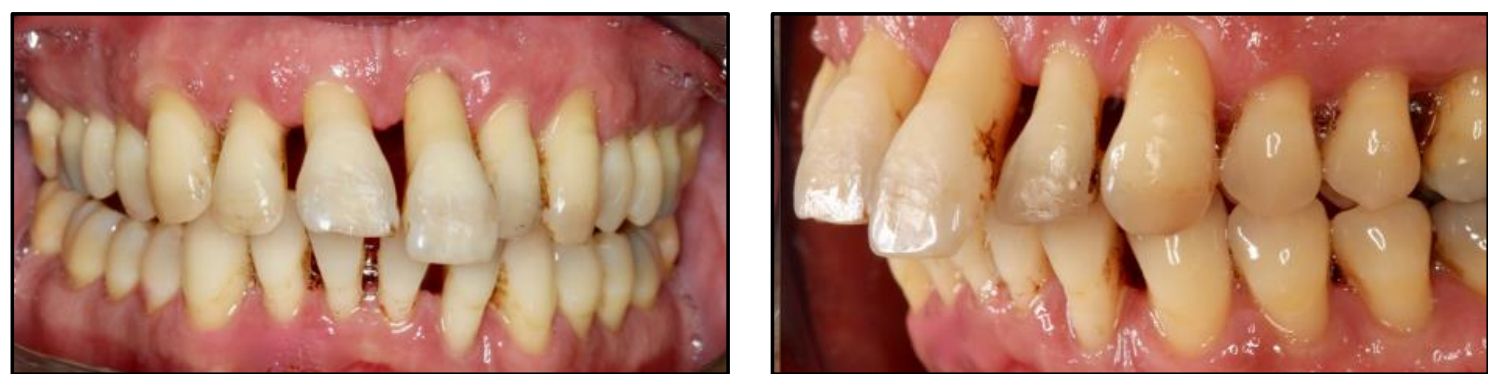

Abbildung 1.6: Frontale (links) und sagittale (rechts) intraorale Fotoaufnahme eines Patienten mit pathologischen Zahnwanderungen und interdentalen Dunkelzonen.

1.3.2 Mehrwert der kieferorthopädischen Therapie im parodontal geschwächten Gebiss

Nach Kessler (1976) ist eine "gute“ Dentition mit idealer Zahnposition, Zahnbogenform und Zahnform mit einem gesunden Parodont vergesellschaftet. Die kieferorthopädische Behandlung kann einen wesentlichen Beitrag zur allgemeinen und speziell funktionellen Rehabilitation des stomatognathen Systems leisten (Gkantidis et al. 2010). Darüber hinaus kann eine kieferorthopädische Behandlung die Möglichkeiten der Parodontaltherapie bei bestimmten Patienten erweitern, indem sie durch eine Optimierung der Zahnstellung zu einer besseren Kontrolle der bakteriellen Besiedlung beiträgt (Carvalho et al. 2018). Kräfte, die potenziell zu nicht gewünschten Bewegungen der Zähne führen, können reduziert werden und schließlich die Gesamtprognose des Gebisses verbessern.

Gingivale Rezessionen können durch Intrusionsbewegungen um bis zu $50 \%$ reduziert werden (Gkantidis et al. 2010; Oh 2011). Darüber hinaus lassen sich Interdentalpapillen orthodontisch rekonstruieren, die aufgrund des knöchernen Einbruches als interdentale Dunkelzonen imponieren (Sharma und Park 2010).

Die Aufrichtung nach mesial gekippter Molaren wird ebenfalls als therapeutische Maßnahme beschrieben. Sie dient der Wiederherstellung der Vertikaldimension, der funktionellen Rehabilitation, sowie einer erleichterten Plaquekontrolle. Ein 
parodontaler Attachmentgewinn und Taschenreduktionen sind die Folge (Diedrich 1989; Gkantidis et al. 2010). Des Weiteren konnte gezeigt werden, dass eine Zahnbewegung in bereits atrophierte Knochenregionen einen Umbau des Alveolarfortsatzes im Sinne eines tissue engineering bewirken kann (Gkantidis et al. 2010).

Zur adäquaten Behandlung dieser klinischen Befunde ist eine sorgfältige interdisziplinäre Planung notwendig (Gkantidis et al. 2010). Bei gründlicher Kontrolle des parodontalen Status und Entzündungsgeschehens kann das parodontale Attachment gesichert und die Prognose der Zähne erheblich verbessert werden (Diedrich 1989; Kirschneck et al. 2017; Gkantidis et al. 2010). Es wird in Einzelfällen von einer Regenerationsfähigkeit des Alveolarknochens berichtet (Xie et al. 2014).

\subsubsection{Beispiel eines Patientenfalls aus der Poliklinik für Kieferorthopädie der Universitätsmedizin Göttingen}

Ein 62-jähriger Patient mit ausgeprägter Parodontitis wurde in der Poliklinik für Kieferorthopädie der Universitätsmedizin Göttingen zur Wiederherstellung der Kaufunktion, beziehungsweise aus parodontalprophylaktischen Gründen vorgestellt. Nach parodontaler Therapie mit Scaling und Wurzelglättung und Aufnahme in ein regelmäßiges Recallprogramm war die orthodontische Korrektur der Zähne angezeigt. Ziel war es, den Langzeiterhalt der Oberkieferfront bei vorangegangenem Knochenverlust zu sichern.

Eine ausführliche kieferorthopädische Befundung folgte. Es zeigte sich ein inkompetenter Lippenschluss, eine Einlagerung der Unterlippe in die deutlich vergrößerte Frontzahnstufe und generalisierter horizontaler Knochenabbau. Die Oberkieferfront stellte sich deutlich aufgefächert in Supraposition, mit einem Diastema mediale und lückiger Verzahnung, dar. Ein overjet von $14,5 \mathrm{~mm}$ und overbite von $10 \mathrm{~mm}$ mit tiefen traumatischen Einbissen wurde befundet.

Zunächst war der Erhalt des Frontzahnes 21 fraglich. Eine Schienung im Oberkiefer wurde prophylaktisch durchgeführt. Anschließend wurde eine Multibracket-Apparatur im Oberkiefer mit maximaler Verankerung an den Molaren 16 und 26 eingegliedert. Außerdem wirkten chirurgische Minischrauben 
regio 13 und 23 zur skelettalen Verankerung unterstützend. Ziel der Therapie war es, die Front zu intrudieren und eine Retrusion zu erreichen. Der Biss sollte in der vertikalen Dimension angehoben werden und gegebenenfalls durch eine konservierende Therapie im Anschluss an die Kieferorthopädie stabilisiert werden. Des Weiteren sollten Lücken verkleinert und das Diastema mediale geschlossen werden. Die abschließende Retention war von besonderer Wichtigkeit und erfolgte sowohl mit einer Schienentherapie als auch über einen oral verklebten Retainer. Es konnten alle Frontzähne erhalten werden und die kieferorthopädische Einordnung war erfolgreich.
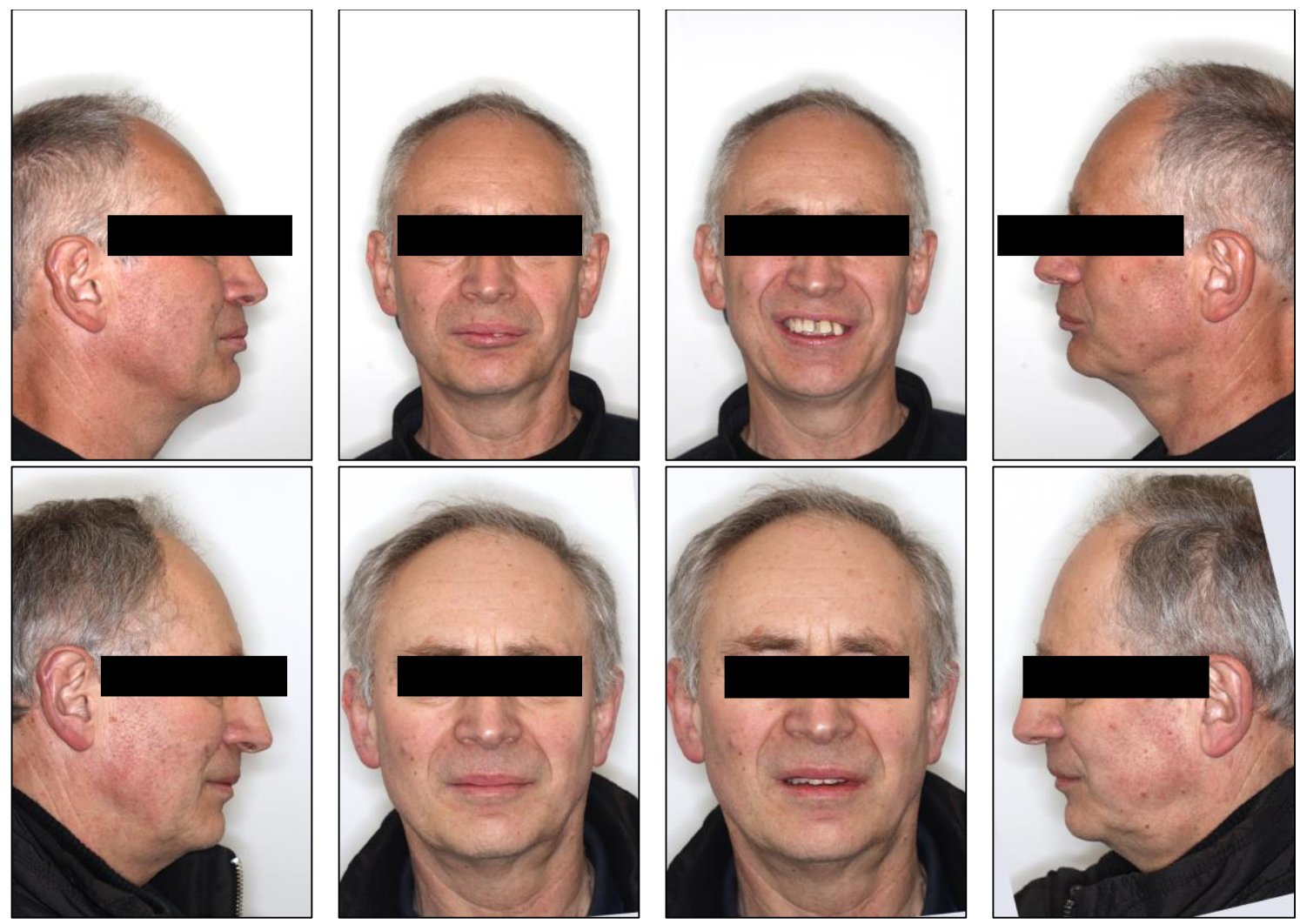

Abbildung 1.7: Fotostat-Aufnahmen vor (obere Zeile) und nach (untere Zeile) der kieferorthopädischparodontologischen Therapie. Beginn der Therapie: 21.09.2016, Ende der Therapie: 21.02.2019 

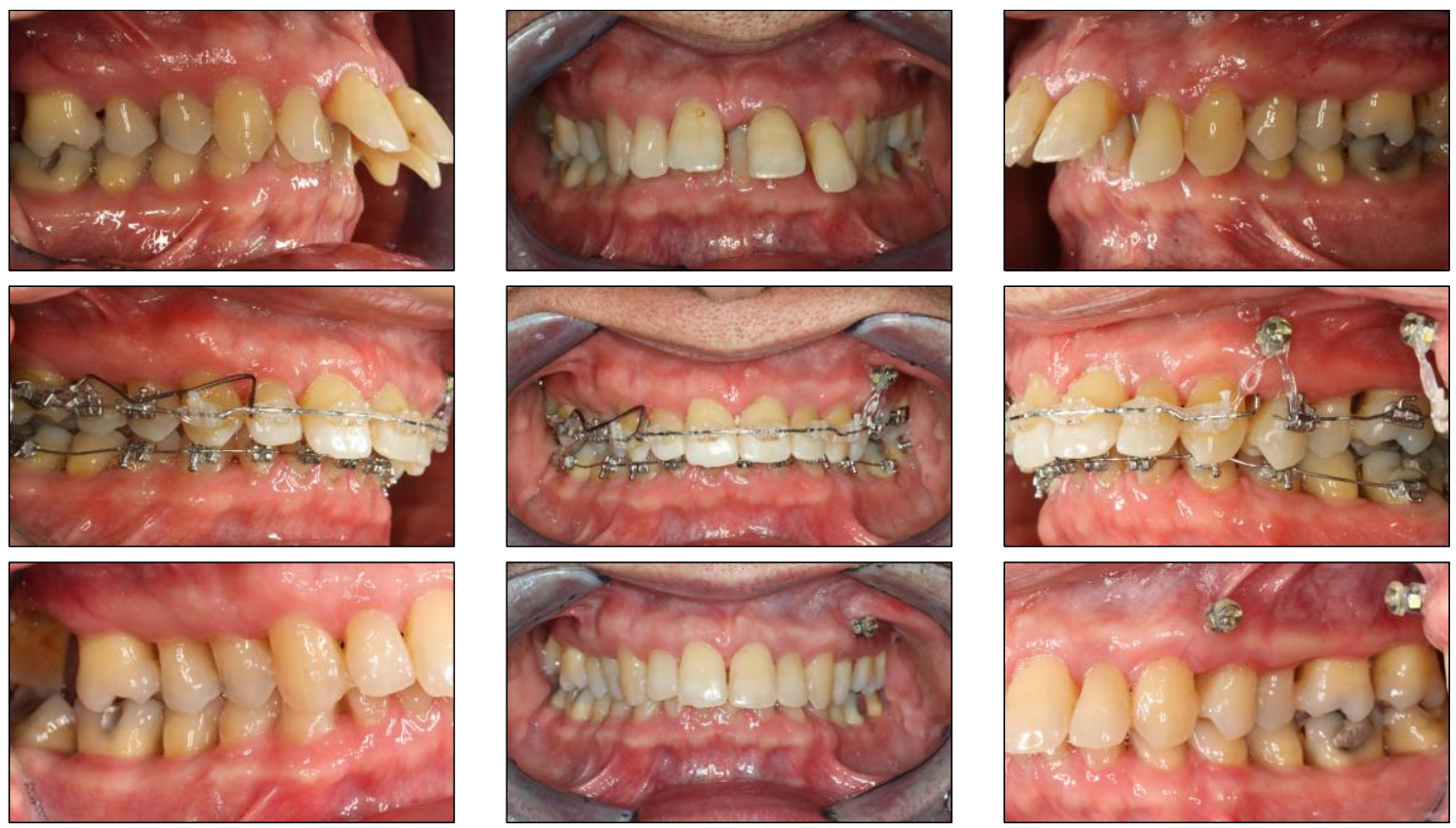

Abbildung 1.8: Intraorale Aufnahmen vor (obere Zeile), während (mittlere Zeile) und nach (untere Zeile) der kieferorthopädisch-parodontologischen Therapie. Oben: 21.09.2016, Mitte: 02.09.2018, Unten: 12.02.2019

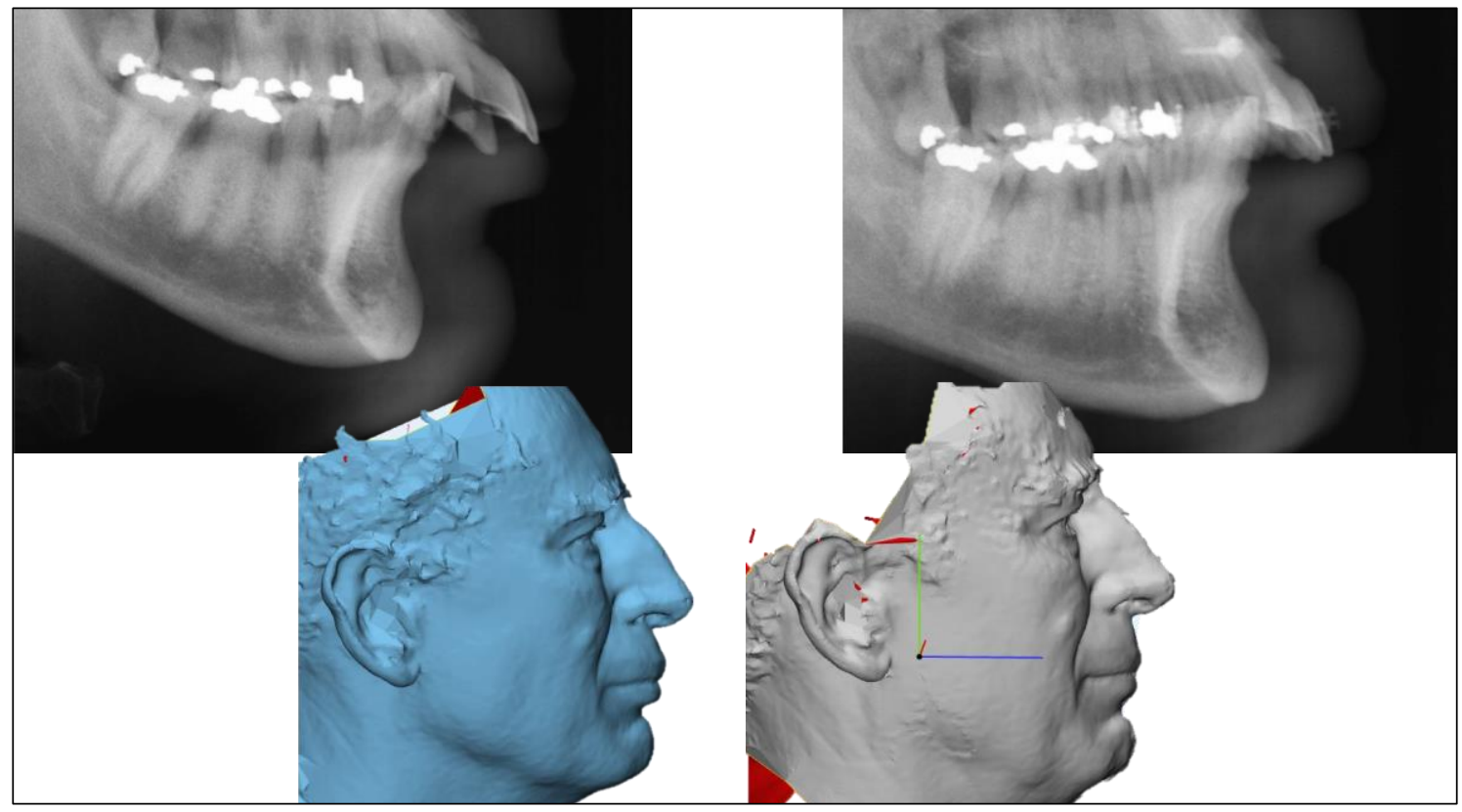

Abbildung 1.9: FRS und dreidimensionaler Oberflächenscan im Profil vor und nach der kieferorthopädischparodontologischen Therapie. 


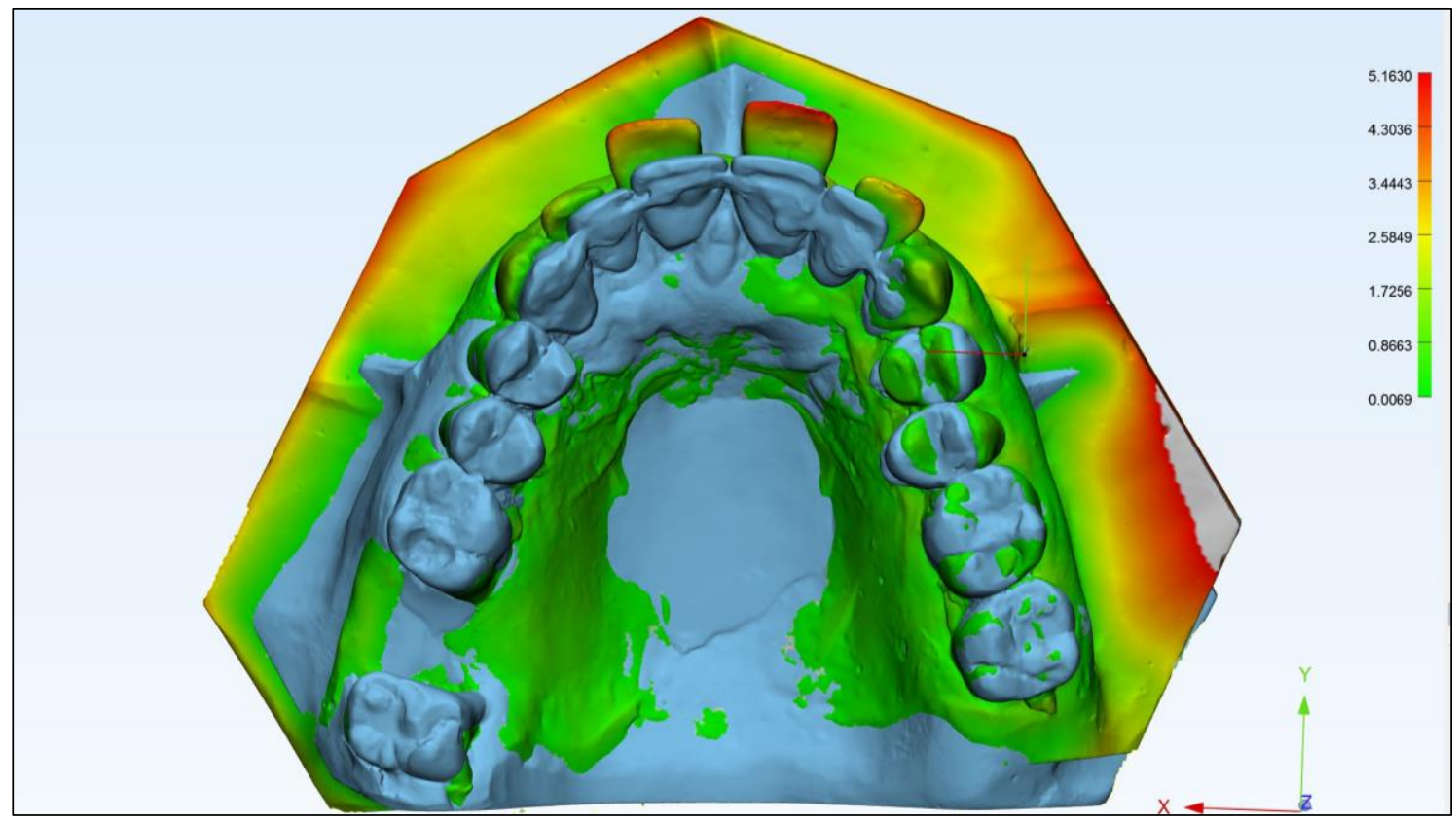

Abbildung 1.10: Dreidimensionale Darstellung eines kieferorthopädisch-parodontologisch kombiniert therapierten Patienten vor Behandlung (bunt) und nach Behandlung (blau).

\subsubsection{Intrusion parodontal kompromittierter Frontzähne}

Zur kieferorthopädischen Behandlung im parodontal geschwächten Gebiss gehört vor allem die in vielen Studien beschriebene Intrusion (Melsen et al. 1989; Cao et al. 2015; Jepsen et al. 2015; Minch et al. 2017). Sie wird vor allem genutzt, um aufgefächerte, labial gekippte und extrudierte Frontzähne in ihre natürliche Position zurückzuführen.

Ziel ist es hierbei, einen physiologischen overjet und overbite herzustellen, um das stomatognathe System aufrechtzuerhalten. Es kommen kontinuierliche leichte, möglichst an der Längsachse des Zahnes ansetzende Kräfte zum Einsatz, um Wurzelresorptionen und Schädigungen des Parodonts zu vermeiden (Diedrich 1989; Melsen et al. 1989; Brunsvold 2005; Gkantidis et al. 2010; Oh 2011; Xie et al. 2014). Ein angebrachter Wert von 0,1 N als Kraft-Dosis-Wirkung scheint dabei effektiv für eine optimale Intrusionen und Regenerationen von Knochendefekten zu sein (Minch et al. 2017).

Die orthodontische Behandlung muss stets in entzündungsfreiem Gewebe stattfinden. Intrusionsbewegungen der Zähne in einem pathologisch besiedelten Gebiss können supragingival gelegene Plaque in eine subgingivale Position verschieben. Dies würde zu weiterer parodontaler Destruktion führen (Ericsson 1986). 
Klinisch und radiologisch konnte gezeigt werden, dass Knochendefekte durch orthodontische Intrusionsbewegungen regenerieren können (Melsen et al. 1989; Jepsen et al. 2015). Das den Zahn umgebende Weichgewebe sowie die interdentale Papille erneuern sich. Außerdem kann die Länge der klinischen Krone reduziert werden. Eine Rehabilitation aller Komponenten (dental / parodontal / gingival / ossär) auf funktioneller Ebene findet statt (Melsen et al. 1989; Cardaropoli et al. 2001; Jepsen et al. 2015). Dabei zeigt sich, dass parodontale Parameter, wie die Sondierungstiefe, der clinical attachment loss und die Rezessionen, nach erfolgreicher kieferorthopädischer Intrusion abnehmen (Cardaropoli et al. 2001; Re et al. 2004; Carvalho et al. 2018).

\subsection{Dreidimensionale Generierung von Oberflächendaten in der Zahnheilkunde}

In der Bildgebung wird allgemein zwischen Volumendaten und reinen Oberflächendaten differenziert. Volumendaten basieren meist auf apparativ aufwendigen Bildgebungstechniken (CT / DVT / MRT), die meist eine Belastung für den Patienten darstellen in Form von Röntgenstrahlung (CT / DVT) oder dem Einsatz von Kontrastmitteln bzw. gegebenenfalls medikamentöser Begleittherapie (MRT). Dagegen können dreidimensionale Oberflächendaten (Gesichtsscans / Modellscans der Zahnbögen etc.) apparativ weniger aufwendig, ohne Röntgenstrahlung und damit für den Patienten weniger belastend durchgeführt werden. Diese Methodik ist zudem auch weniger zeitaufwendig.

Darüber hinaus bieten dreidimensionale Darstellungen von Oberflächen weicher und harter Gewebe eine Fülle neuer Daten, anhand derer der behandelnde Arzt Befunde erheben und Therapien planen kann (Solem 2017). Seit Anfang des 20. Jahrhunderts gibt es die Bemühungen, dreidimensionale Modelle des kompletten Gesichts zu generieren, wobei sich die Dentition in der anatomisch korrekten Position befindet (Rangel et al. 2008). Ab den 1980er Jahren wurden erstmals dreidimensionale Aufnahmetechniken erprobt.

Kawano (1987) verwendete in seiner Studie die Moiré Topography, welche sich an Konturlinien auf einem Objekt orientiert, Moss et al. (1988) nutzten die Computertomographie, die Laser-Scanning Methode wurde von McCance et al. (1992) etabliert und Ayoub et al. (1996) führten neue Aspekte der 
Stereofotogrammmetrie in der Zahn-Mund-Kieferheilkunde ein. Heutzutage wird die zuletzt genannte Methodik bereits häufig in Kliniken und im täglichen Praxisalltag angewandt. Vorteil dabei ist, dass die Oberflächenbildgebung komplett ohne die Verwendung von Röntgenstrahlen auskommt und somit noninvasiv, schnell und beliebig oft wiederholbar ist (Rangel et al. 2008; Rosati et al. 2012; Masoud et al. 2017).

Unter dem Begriff Stereofotogrammmetrie versteht man ein Verfahren zur Bildmessung, bei dem mehrere Aufnahmen aus verschiedenen Richtungen erstellt werden. Diese werden in ihrer genauen Form und Lage bestimmt und anschließend zu einem Objekt zusammengefügt.

Das Verfahren der Stereofotogrammmetrie wird unter anderem in der dreidimensionalen Analyse der Gesichtsoberfläche genutzt. Es ist dadurch möglich, das Lippenprofil in Bezug zur Zahnstellung zu beurteilen (Sawyer et al. 2009; Kim et al. 2014; Kuhn et al. 2016). Es konnten Korrelationen zwischen der Frontzahnstellung und der Lippenmorphologie gezeigt werden. Mit zunehmender Protrusion der Zähne bewegt sich die Lippe nach oben und vorne (Kim et al. 2014)

1.4.1 Pathologische Weichteilveränderungen aufgrund von Zahnfehlstellungen Veränderungen des Weichteilprofils korrelieren stark mit Zahnstellungsänderungen. Bislang existieren zahlreiche Fernröntgenseitenbild (FRS)-Studien zu dieser Thematik. Allerdings spiegelt eine zweidimensionale Betrachtung zwischen Zahnstellung und Weichteilprofil, wie sie das FRS ermöglicht, die Komplexität der Relation nicht wider. Die Quantifizierung sollte auf Basis dreidimensionaler Datensätze mit Integration einer dreidimensionalen Analyse, beispielsweise durch das Setzen von landmarks, erfolgen. Diese Methodik ermöglicht es, Unterschiede zu anderen Patienten oder Verlaufskontrollen desselben Patienten zu erstellen (Solem 2017).

Studien zeigten signifikante Zusammenhänge zwischen der Oberkieferfrontzahnbewegung und Veränderungen der Lippenmorphologie (Kuhn et al. 2016). Damit einhergehen funktionelle und ästhetische Verbesserungen, die durch die Korrektur der Zahnstellung eine Veränderung der Lippenposition und des umgebenden perioralen Weichgewebes bewirken. 
Studien zur Simulation von Bewegungen der Oberkieferfrontzähne konnten zeigen, dass die Positionsänderungen des Weichgewebes in Abhängigkeit von Zahnbewegungen vorhersagbar sind. Analysen auf Basis von Stereofotogrammmetrie in Form von Abstandsmessungen zwischen Dentition und Weichteil werden hierbei genutzt (Kim et al. 2014).

Zukünftig könnten Erkenntnisse über diese Zusammenhänge als Orientierungshilfen bei der kieferorthopädischen Behandlung dienen, um ein funktionelles / dentofaziales Gleichgewicht zu erreichen (Ceinos et al. 2017). 


\subsection{Ziele der Arbeit}

Pathologische Zahnwanderungen im parodontal geschädigten Gebiss lassen sich aktuell bei Erwachsenen ( $\geq 40$ Jahre) mit einer Prävalenz von $>50 \%$ nachweisen und zeigen eine steigende Inzidenz. Demnach kommt diesem Patientenklientel zukünftig hohes wissenschaftliches und klinisches Interesse für parodontal-kieferorthopädische Aspekte zu.

Die Ursachen pathologischer Zahnwanderungen werden dabei auf Basis zahlreicher Faktoren diskutiert. Ein Hauptfaktor scheint der Verlust einer ausreichenden parodontalen Unterstützung im Alveolarfortsatz der Zähne zu sein (Martinez-Canut et al. 1997; Towfighi et al. 1997; Brunsvold 2005). Bisher wurde jedoch die potentielle Interaktion zwischen der Zahnfehlstellung und dem Grad der parodontalen Destruktion unzureichend untersucht. Zusätzlich existiert bislang keine Möglichkeit die charakteristischen Zahnfehlstellungen im Kontext der perioralen Gesichtsweichteile anhand eines dreidimensionalen Patientenmodells zu analysieren. Darüber hinaus fehlen Daten über die Behandlungsbedürftigkeit dieser Patienten, sowie Daten über den Zustand der Mundgesundheit bzw. Aufklärung über Behandlungsmöglichkeiten dieser Patienten.

Daher wurden folgende Ziele formuliert:

Klinisch:

Es sollte ein Zusammenhang zwischen pathologischen Zahnwanderungen und der parodontalen Destruktion, genauer des clinical attachment loss (CAL), in der Frontzahnregion beim älteren erwachsenen kieferorthopädischen Patienten untersucht werden.

Bildgebung:

Ein dreidimensionales virtuelles Patientenmodell sollte anhand einer Oberflächentriangulation der Gesichtsweichteile und der Zahnbögen generiert werden.

Behandlungsbedürftigkeit:

Eine kieferorthopädische Behandlungsbedürftigkeit und der Behandlungswunsch sowie die mundgesundheitsbezogene Lebensqualität bei älteren erwachsenen Patienten sollte im Hinblick auf die parodontale Destruktion quantifiziert werden. 


\section{Material und Methoden}

Die vorliegende explorative Querschnittstudie wurde von der institutionellen Ethik-Kommission der Universitätsmedizin Göttingen (Antragsnummer 3/1/17) genehmigt. Sie wurde gemäß der Deklaration von Helsinki durchgeführt. Alle Patienten nahmen freiwillig an dieser Studie teil. Sie wurden vor Beginn der Studie umfassend über die Ziele und den Ablauf informiert. Die Einverständniserklärung erfolgte schriftlich.

\subsection{Rekrutierung des Patientenkollektivs}

Die Patienten wurden zwischen Februar 2017 und März 2018 im Rahmen eines interdisziplinären Therapieregimes aus der Poliklinik für Präventive Zahnmedizin, Parodontologie und Kariologie rekrutiert. Es wurden alle Patienten anhand des vorhandenen Befundbogens und röntgenologischen Übersichtsaufnahmen ausgeschlossen, welche aufgrund der Dentition nicht den Einschlusskriterien der Studie entsprachen (siehe unten).

\subsubsection{Einschlusskriterien}

Für die Studie wurden Patienten der Poliklinik für Präventive Zahnmedizin, Parodontologie und Kariologie mit einem Alter von $\geq 40$ Jahre zugelassen, deren schriftliches Einverständnis für die Studie vorlag. Es musste eine vollständige Bezahnung in der Oberkieferfront und Unterkieferfront von Eckzahn zu Eckzahn vorhanden sein sowie die Abwesenheit eines dentalen Traumas in der Patientenhistorie.

\subsubsection{Ausschlusskriterien}

Ein fehlendes Einverständnis zur Teilnahme an der Studie führte zum Ausschluss. Außerdem wurden Patienten mit einem anterior offenen Biss und anteriorem Kreuzbiss ausgeschlossen. Ein angeborenes Syndrom, wie z. B. eine Lippen-Kiefer-Gaumenspalte, wurde ebenfalls als Ausschlusskriterium definiert. 


\subsection{Datenerhebung - klinisch parodontale Parameter}

Alle Patienten wurden in der Poliklinik für Kieferorthopädie der Universitätsmedizin Göttingen von demselben Behandler untersucht. Die Daten wurden in einem individualisierten Befundbogen notiert und anschließend ausgewertet. Es wurden Messungen im Ober- und Unterkiefer jeweils an den sechs Frontzähnen vorgenommen. An den Zähnen 13 bis 23 und 33 bis 43 wurden je sechs Messpunkte (mesial, mittig und distal an der labialen und oralen Zahnfläche) untersucht. Insgesamt wurden an 72 Messpunkten mit einer Parodontalsonde die Sondierungstiefen (ST) und Rezessionen (RZ) erhoben. Beide Werte wurden addiert, um den clinical attachment loss (CAL) zu ermitteln. Dieser beschreibt die Distanz zwischen der Schmelz-Zementgrenze und dem klinisch sondierbaren Boden der jeweiligen parodontalen Tasche.

Für jeden Zahn wurde zusätzlich als ordinal orientierter Parameter der Lockerungsgrad bestimmt (Lange 1981). Dies wurde unter Zuhilfenahme des Zeigefingers und eines Instrumentengriffes durchgeführt.

Tabelle 2.1: Definition des Lockerungsgrades

\begin{tabular}{ll}
\hline Lockerungsgrad & Definition \\
\hline Grad 0 & Krone nicht sichtbar auslenkbar \\
Grad I & Krone $\leq 1 \mathrm{~mm}$ auslenkbar \\
Grad II & Krone $>1 \mathrm{~mm}$ auslenkbar \\
Grad III & Krone beweglich auf Lippen- und Zungendruck und / oder in \\
& axialer Richtung \\
\hline
\end{tabular}

Des Weiteren wurden Situationsabformungen des Oberkiefers und Unterkiefers mit dem Alginat Tetrachrom (KANIEDENTA GmbH \& Co. KG Dentalmedizinische Erzeugnisse, Herford) genommen und anschließend mit Spezial-Hartgips (Picodent, pico-crema® soft, Dental-Produktions- und Vertriebs-GmbH, Wipperfürth) ausgegossen. Die habituelle Bisslage wurde mit Regidur® i (Bisico, Bielefelder Dentalsilicone $\mathrm{GmbH} \&$ Co. KG, Bielefeld) registriert. 


\subsubsection{Definition der Patientengruppen}

Die Patienten wurden entsprechend ihres Ausprägungsgrades der parodontalen Destruktion eingeteilt. Basierend auf der klinischen Definition der CDC Working Group (Center for Disease Control and Prevention) der American Association of Periodontology erfolgte die Einteilung der Patienten in drei Gruppen. In der Falldefinition der CDC Working Group wurden nur die dritten Molaren ausgeschlossen. In der vorliegenden Studie wurde die Definition auf das Frontzahnsegment von Eckzahn bis Eckzahn angewandt. Die Falldefinition für eine schwere Parodontitis erfordert zwei oder mehr interproximale Messpunkte an verschiedenen Zähnen mit einem CAL $\geq 6 \mathrm{~mm}$ im Front-Eckzahnbereich und einen oder mehrere Messpunkte mit einer ST von $\geq 5 \mathrm{~mm}$. Um eine moderate Parodontitis handelt es sich, wenn zwei oder mehr interproximale Messpunkte an verschiedenen Zähnen einen CAL von $\geq 4 \mathrm{~mm}$ aufweisen oder an zwei oder mehr interproximalen Stellen eine ST von $\geq 5 \mathrm{~mm}$ gemessen werden. Treffen die Definitionen für eine schwere und moderate Parodontitis nicht zu, liegt eine Nichterkrankung oder schwache Parodontitis vor, bei der der CAL $<4 \mathrm{~mm}$ und die ST bei $<5 \mathrm{~mm}$ liegen (Page und Eke 2007). 


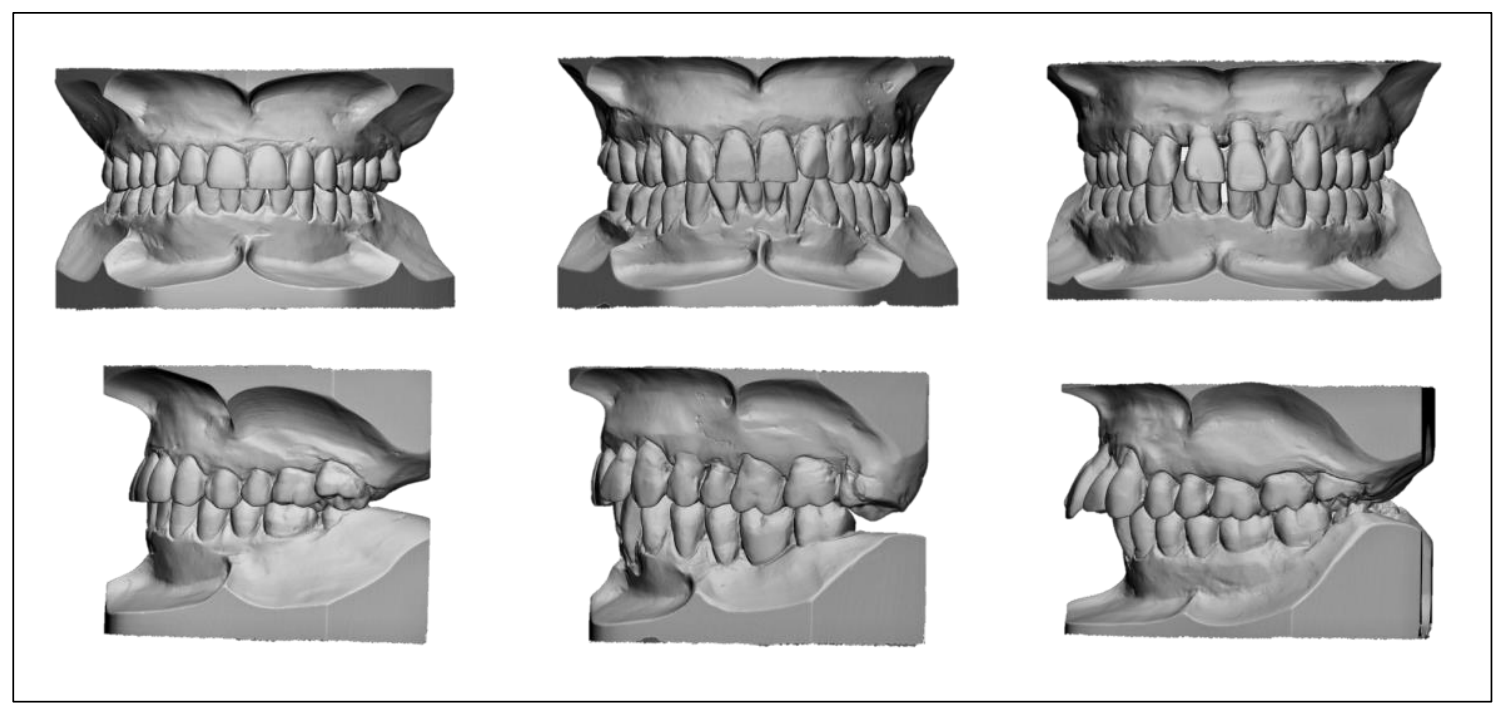

Abbildung 2.1: Dreidimensionale Modelle in Frontansicht (obere Zeile) und sagittaler Ansicht (untere Zeile) je eines Patienten ohne parodontale Erkrankung (links), mit moderater Parodontitis (zentral), mit schwerer Parodontitis (rechts).

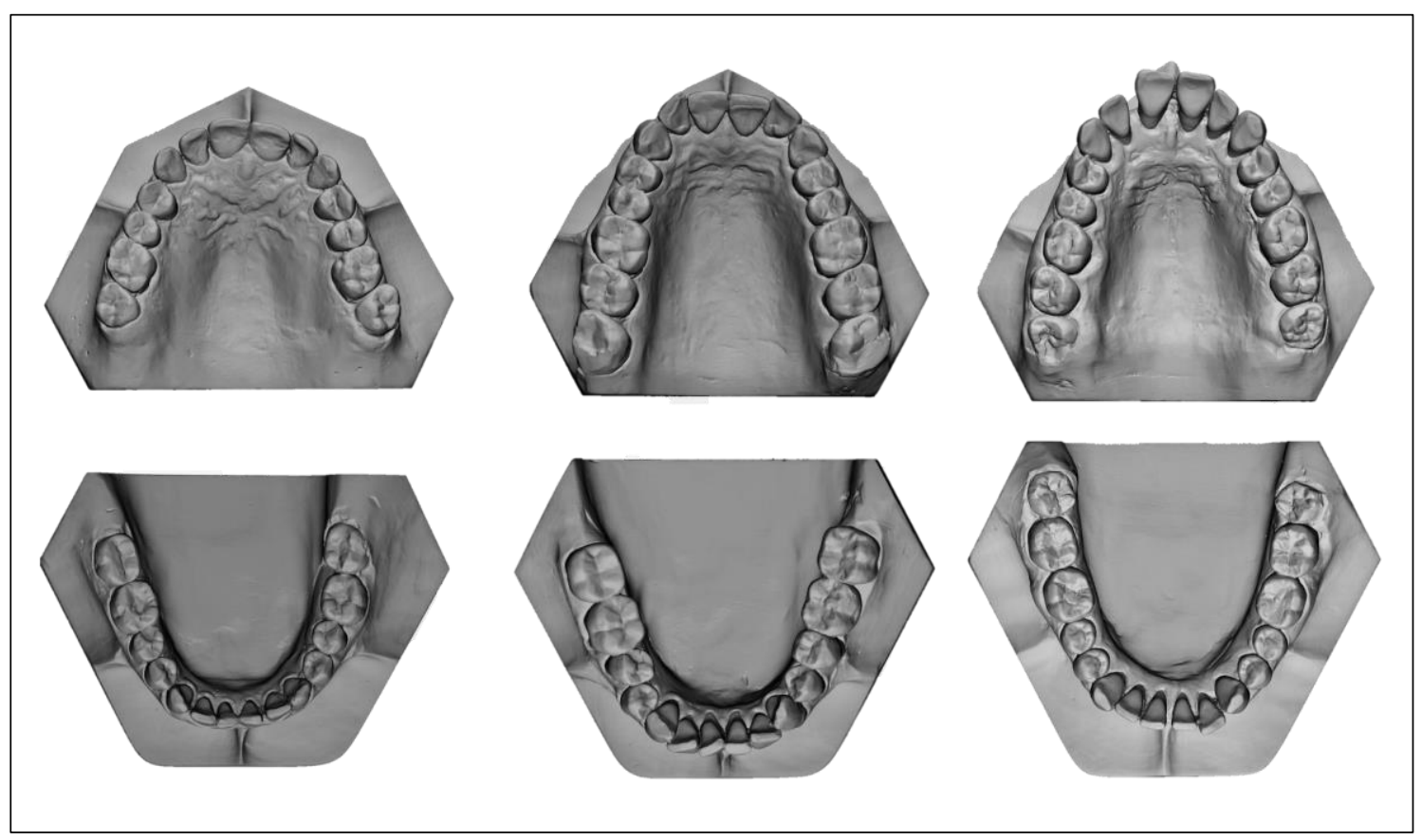

Abbildung 2.2: Dreidimensionale Modelle in der Aufsicht des Ober- (obere Zeile) und Unterkiefers (untere Zeile) je eines Patienten ohne parodontale Erkrankung (links), mit moderater Parodontitis (zentral), mit schwerer Parodontitis (rechts). 


\subsection{Modellvermessung - orthodontische Parameter}

Die Gipsmodelle wurden zunächst dreidimensional in habitueller Interkuspidation getrimmt und auf orthodontische Parameter mit einem millimeter-skalierten Messschieber mit Nonius Ablesung ausgewertet. Gemessen wurden folgende Parameter (siehe Tabelle 2.2), wobei jeweils das größte Ausmaß des jeweiligen Parameters festgesetzt wurde.

Tabelle 2.2: Definition der Modellvermessung

\begin{tabular}{|c|c|c|}
\hline \# & Orthodontische Parameter & Definition \\
\hline 1 & Overjet & $\begin{array}{l}\text { sagittale Frontzahnstufe }(\mathrm{mm}) \text { zur Okklusionsebene an } \\
\text { der am stärksten ausgeprägtesten Stelle gemessen }\end{array}$ \\
\hline 2 & Overbite & $\begin{array}{l}\text { vertikale Frontzahnstufe ( } \mathrm{mm}) \text { an der am stärksten } \\
\text { ausgeprägtesten Stelle gemessen }\end{array}$ \\
\hline 3 & Little-Index in OK / UK & $\begin{array}{l}\text { lineare Abstände zwischen den anatomischen } \\
\text { Kontaktpunkten }(\mathrm{mm}) \text { jedes Inzisivus zu den jeweiligen } \\
\text { entsprechenden Punkten seiner Nachbarzähne gemessen }\end{array}$ \\
\hline
\end{tabular}

Der overjet beschreibt die sagittale Frontzahnstufe und wird an der am stärksten ausgeprägten Stelle des Frontzahnsegments in Millimeter gemessen (Fisk 1960) (Abbildung 2.3.a). Der overbite beschreibt die vertikale Frontzahnstufe und wird auch an der am stärksten ausgeprägten Stelle des Frontzahnsegments in Millimeter bestimmt (McCall 1944) (Abbildung 2.3.b). Um den Schweregrad der Malokklusion zu quantifizieren, wurde der Little-Index erhoben (Little 1975). Dabei wurden die linearen Abstände zwischen den anatomischen Kontaktpunkten jedes Inzisivus zu den jeweiligen entsprechenden Punkten seiner Nachbarzähne gemessen. Der Messschieber wurde dabei parallel zur Okklusionsebene ausgerichtet (Abbildung 2.3.c). Durch diesen Wert konnte eine Aussage darüber getroffen werden, wie groß die tatsächliche Entfernung ist, die die anatomischen Kontaktpunkte aufeinander zu bewegt werden müssten, um eine ideale Zahnreihe zu erhalten. Little (1975) beschrieb die Methodik ursprünglich für das Unterkieferfrontzahnsegment. 
In dieser Studie wurde die Methode ebenfalls auf das Oberkieferfrontzahnsegment übertragen. Anschließend wurden die Einzelwerte addiert und so eine Summe für die Irregularität der anatomischen Kontaktpunkte für den Oberkiefer und den Unterkiefer gebildet.

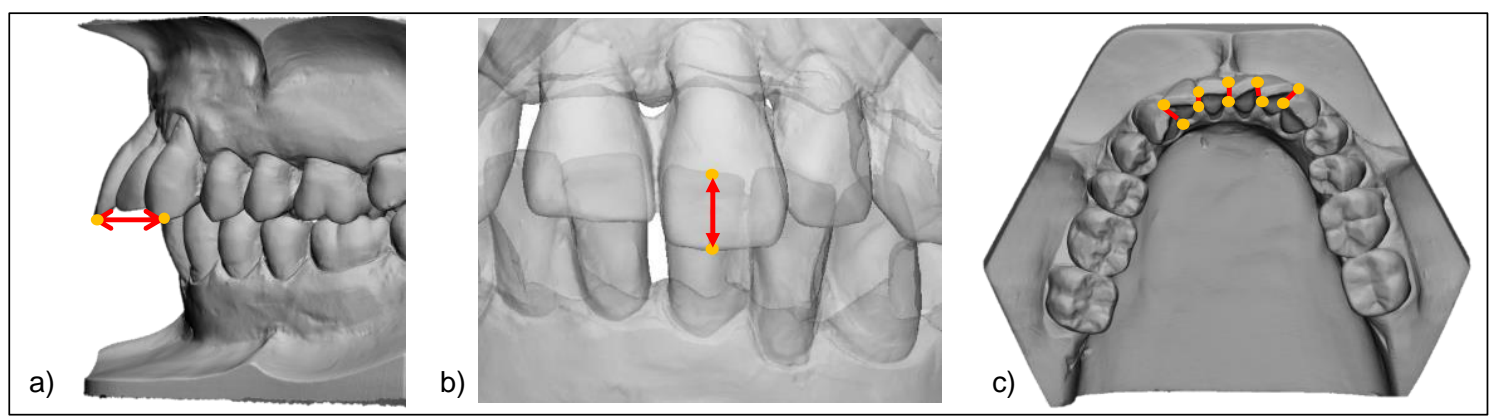

Abbildung 2.3: Dreidimensionale Darstellung des overjets (a), overbites (b) und Little-Index hier im Unterkiefer (c).

\subsection{Dentofaziales Modell}

Zur Analyse der Frontzahnstellung im Verhältnis zur Lippenmorphologie der Patientendaten im Hinblick auf den kieferorthopädischen Behandlungsbedarf, welcher sich in Frontzahndisharmonien, einer vergrößerten sagittalen / vertikalen Stufe, pathologischen Weichteilveränderungen und Komponenten der Ästhetik äußert, wurde ein virtuelles Patientenmodell mit der anatomisch individuellen Positionierung des Oberkiefers und Unterkiefers zu den Gesichtsweichteilen erstellt. Alle Patientendaten wurden dreidimensional erfasst. Anschließend konnte eine dreidimensionale Analyse der Frontzahnstellung zum Gesichtsweichteil durchgeführt werden.

\subsubsection{Erstellung des virtuellen Patientenmodells}

Für eine standardisierte Analyse wurden die analogen Gipsmodelle mit einem optischen Streifenlichtscanner digitalisiert (siehe Abbildung 2.4). Der Scanner S300 Ortho der Firma Zirkonzahn (Zirkonzahn S.R.L., Gais, Italien) hat eine Scanpräzision von $\leq 10 \mu \mathrm{m}$. Über die Funktion „Quick Reg“ wurden zunächst beide Modelle getrennt voneinander eingescannt und anschließend über einen Vestibulärscan in maximaler Interkuspidation mit der „Scan \& Match“ Funktion überlagert. Somit wurden Daten im STL-Format erzeugt. 
Die Gesichtsweichteile wurden mittels lichtoptischer Verfahren mit dem Vectra ${ }^{\circledR}$ N5 Head Scanner (Version 7.4.1, Canfield Scientific, Parsippany, NJ, USA) erfasst. Dieser wurde durch eine wöchentliche Kalibrierung auf seine Genauigkeit geprüft. Von jedem Patienten wurden zwei dreidimensionale Scanaufnahmen der Gesichtsregion angefertigt: 1.) in Lippenschlusslage; 2.) mit Wangenabhalter, um die Zahnreihen in habitueller Okklusion darzustellen.

Anhand gescannter Ober- und Unterkiefer Gipsmodelle wurde die Position der Dentition in dem erhobenen dreidimensionalen Weichteildatensatz ermittelt. Die Zusammenhänge zwischen Weichteilanatomie und Dentition wurden dargestellt. Ein dreidimensionaler Scan besteht aus 5 einzelnen, aus verschiedenen Positionen gleichzeitig aufgenommenen Fotos. Eine Aufnahme von fünf Fotos erfolgte in durchschnittlich 1,5 ms. Diese wurden anschließend computerbasiert zu einem dreidimensionalen Bild zusammengesetzt. Ein dreidimensionaler Scan erfolgte bei geschlossenem und entspanntem Mund. Der Andere wurde mit Wangenabhaltern durchgeführt, welche durch den Behandler positioniert und von dem Patienten während der Aufnahme gehalten wurden.

Um die Aufnahmen zu optimieren, wurden die Zahnoberflächen mit dem Mattierungsmittel CEREC Optispray (Sirona Dental Systems GmbH, Bensheim) besprüht. Die Scans lagen anschließend ebenfalls im STL-Dateiformat vor. 


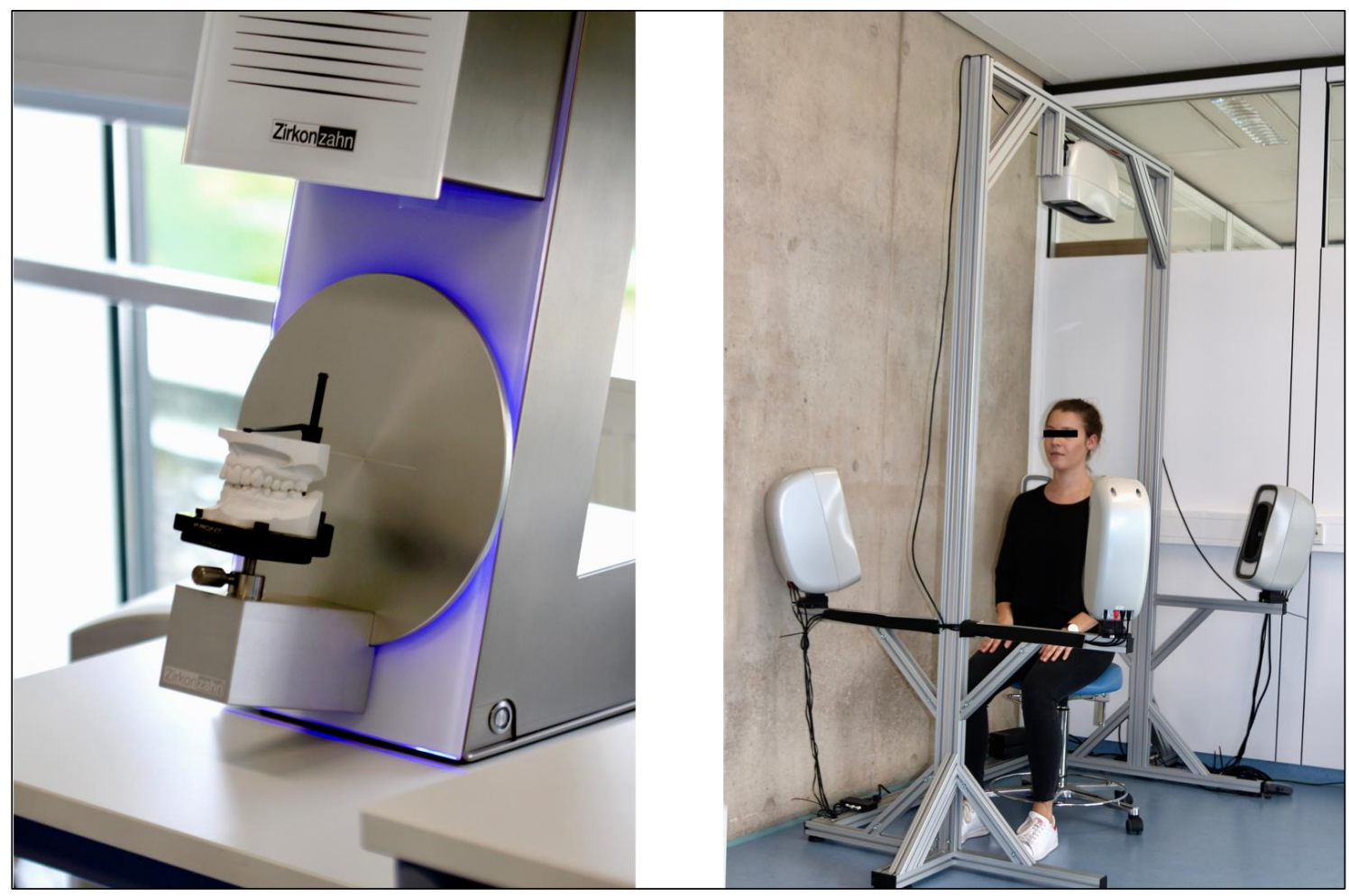

Abbildung 2.4: Scanner S300 Ortho der Firma Zirkonzahn zur Digitalisierung der analogen Gipsmodelle (links), Vectra ${ }^{\circledR}$ N5-Head-Scanner zur lichtoptischen Erfassung der Gesichtsoberfläche (rechts).

\subsubsection{Erstellung des dreidimensionalen Patientenmodells}

Zur Erstellung des dreidimensionalen Patientenmodells wurden der Modellscan und die beiden Weichteilscans, sowohl mit Lippenschluss als auch mit Wangenabhaltern, in dem Programm 3-matic Research 13.0 in Anlehnung an Rangel et al. (2008) überlagert. Hierzu wurde eine N-Point Registration über das Setzen von vier Punkten durchgeführt, um die dreidimensionalen Modelle zueinander im Raum zu orientieren. Der Modellscan wurde zunächst in einer Registrierung I zu dem Weichteilscan mit Wangenabhaltern orientiert und anschließend über eine globale Registrierung feineingestellt. Eine stetige Annäherung der Objekte zueinander bis zu einem stagnierenden Abstandswert wurde durchgeführt. Hierzu wurde der „Distance threshold“ von $2 \mathrm{~mm}$ auf 0,3 mm reduziert, um die Objekte fortwährend einander anzunähern. Der „Distance threshold“ ist so definiert, dass sobald der Abstand zwischen einem Punkt auf dem fixierten Objekt und dem bewegten Objekt größer als der Schwellenwert ist, die Punkte während der Registrierung nicht verwendet werden. Ergebnis der ersten Registrierung war eine virtuelle Darstellung des Modellscans und Weichteilscans mit Wangenabhaltern in korrekter anatomischer Position zueinander (Abbildung 2.5). 


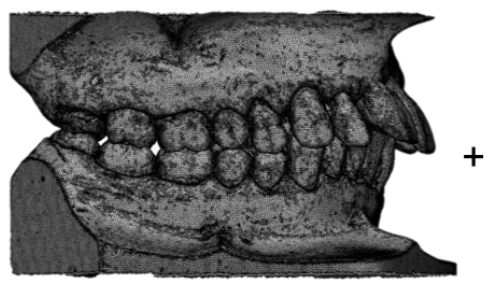

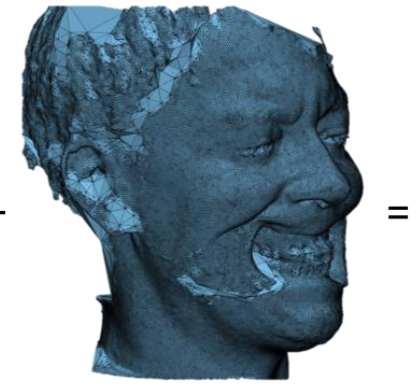

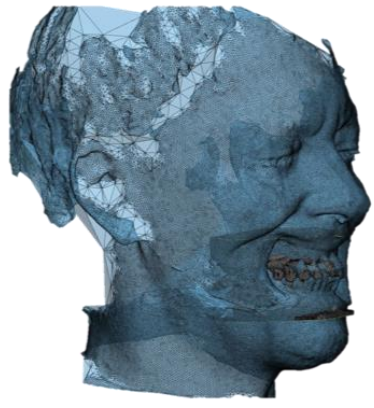

$a b$

Abbildung 2.5: Registrierung I: Überlagerung der dreidimensionalen Datensätze - Modellscan (a) überlagert mit Weichteilscan mit Wangenabhaltern (b) ergibt das anatomisch korrekte virtuelle Patientenmodell ohne komplettes Weichteil (ab).

Anschließend wurde eine Registrierung II durchgeführt mit gleichem Procedere. Allerdings wurde hierfür der Weichteilscan mit Wangenabhaltern und der Weichteilscan mit geschlossenem Mund überlagert und der Modellscan als fixiertes Objekt mitbewegt. Ergebnis ist das komplette anatomische und virtuelle Modell eines Patienten mit der Dentition in korrekter Position (Abbildung 2.6). Durch Ausblenden des Weichteilscans mit Wangenabhaltern entsteht ein dreidimensionales Modell, bestehend aus der Dentition, die in das umgebende Weichgewebe ohne störende Artefakte durch die Wangenabhalter eingebettet ist. Im Folgenden können Messungen, welche das Weichteil und die Dentition berücksichtigen, durchgeführt werden.

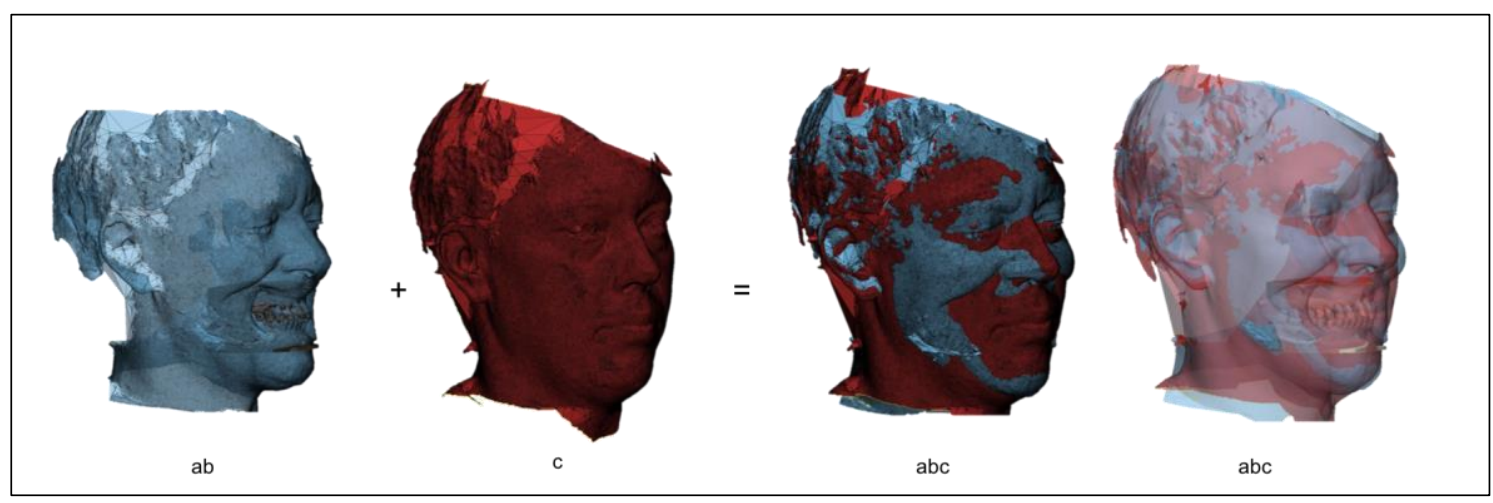

Abbildung 2.6: Registrierung II: Überlagerung der dreidimensionalen Datensätze - Virtuelles Modell aus Modellscan und Weichteilscan mit Wangenabhaltern (ab) überlagert mit Weichteilscan in Okklusion (c) ergibt das komplette anatomisch korrekte Patientenmodell (abc). 
Zur Vermessung des dreidimensionalen Patientenmodells wurde ein individuelles Koordinatensystem programmiert. Die Horizontalebene wurde als natural head position (NHP), welche durch die bilateralen Exocanthions führt, definiert (Dvortsin et al. 2011). Senkrecht zu der Horizontalebene wurde die Sagittalebene aufgespannt. Diese hat als Referenz das Weichteilnasion und den Punkt Subnasale. Die Koronalebene wurde aufgespannt, indem eine Orthogonale zur Horizontalebene und Sagittalebene, welche durch das Weichteilnasion führt, definiert wurde. Als Ursprung des neuen Koordinatensystems wurde das Weichteilnasion definiert. Die axiale, sagittale und koronale Referenzebene wurden als $\mathrm{x}$-, $\mathrm{y}$ - und z-Achse beschrieben. Die Richtungen nach links, nach oben und nach anterior wurden für die jeweiligen Achsen als positiv betrachtet ( $\mathrm{Kim}$ et al. 2014; Li et al. 2019). Um das Weltkoordinatensystem mit dem Objektkoordinatensystem gleichzusetzen, wurde ein „Plane to Plane Align“ durchgeführt. Dieser wurde über die xy-Ebene des Weltkoordinatensystems und die xy-Ebene des Objektkoordinatensystems angewandt und alle weiteren Objekte in dem Projekt wurden mitbewegt.

Die Überlagerung der dreidimensionalen Daten wurde nach einem Zeitraum von einer Woche erneut durchgeführt und der Abstand zwischen den Datensätzen bestimmt, um die Genauigkeit der Überlagerung zu bestimmen. 


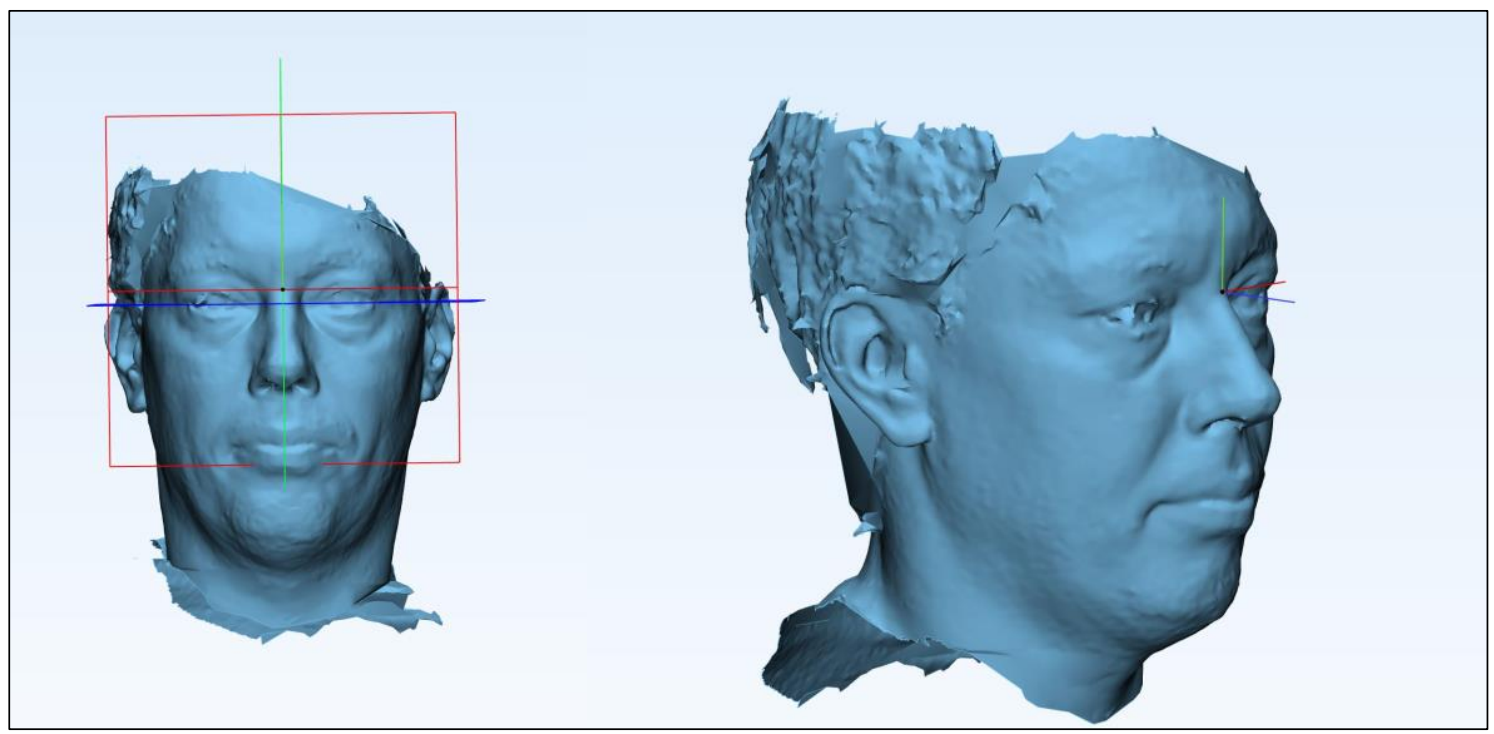

Abbildung 2.7: Definition der axialen (blau), sagittalen (grün) und koronalen (rot) Referenzebenen (links), aufgespanntes Koordinatenkreuz (rechts).

\subsubsection{Vermessung des dreidimensionalen Patientenmodells}

Die Vermessung des dreidimensionalen Patientenmodells wurde anhand eines neu programmierten, individuellen Koordinatensystems, welches als Ursprung das Weichteilnasion hat, durchgeführt. Folgende Punkte wurden definiert, anschließend vermessen und in Bezug gesetzt (Tabelle 2.3, Tabelle 2.4, Tabelle 2.5). 
Tabelle 2.3: Definition der anatomischen Messpunkte.

\begin{tabular}{|c|c|c|}
\hline & „Landmarks“ & \\
\hline \# & Anatomische Punkte & Definition \\
\hline 1 & Nasion $(N)$ & $\begin{array}{l}\text { Anteriorster Punkt der Sutura naso-frontalis übertragen } \\
\text { auf das Weichteilprofil }\end{array}$ \\
\hline 2 & Subnasale (Sn) & Posteriorster Punkt der nasolabialen Kurvatur \\
\hline 3 & Exocanthion re. (Ecr) & $\begin{array}{l}\text { Weichteilpunkt an der äußersten Kommissur des rechten } \\
\text { Auges }\end{array}$ \\
\hline 4 & Exocanthion li. (Ecl) & $\begin{array}{l}\text { Weichteilpunkt an der äußersten Kommissur des linken } \\
\text { Auges }\end{array}$ \\
\hline 5 & Nasenspitze (Nspitze) & Anteriorster Punkt der Nasenspitze in y-Achsenrichtung \\
\hline 6 & Kinn (K) & $\begin{array}{l}\text { Anteriorster Punkt des Weichteil-Kinn-Profils } \\
\text { (Weichteilpogonion) }\end{array}$ \\
\hline 7 & labium superius (LS) & Mittelpunkt des Kupidobogens \\
\hline 8 & labium inferius (LI) & Mittelpunkt der Lippen Rot-Weiß Grenze der Unterlippe \\
\hline 9 & $\mathrm{R}^{1} \frac{1}{2} \mathrm{Chp}$ & $\begin{array}{l}\text { Mittelpunkt zwischen rechter Crista philtri und rechtem } \\
\text { Cheilion }\end{array}$ \\
\hline 10 & RChp & $\begin{array}{l}\text { Punkt auf dem rechten oberen erhabenen Punkt des } \\
\text { Philtrums }\end{array}$ \\
\hline 11 & LChp & $\begin{array}{l}\text { Punkt auf dem linken oberen erhabenen Punkt des } \\
\text { Philtrums }\end{array}$ \\
\hline 12 & L1/2Chp & $\begin{array}{l}\text { Mittelpunkt zwischen linker Crista philtri und linkem } \\
\text { Cheilion }\end{array}$ \\
\hline 13 & Mittelpunkt Lippenschlusslinie (ML) & $\begin{array}{l}\text { Mittelpunkt der Lippenkurvatur zwischen Ober- und } \\
\text { Unterlippe }\end{array}$ \\
\hline 14 & Inzisalkante 12 (I12) & Mittelpunkt der Inzisalkante an 12 \\
\hline 15 & Inzisalkante 11 (I11) & Mittelpunkt der Inzisalkante an 11 \\
\hline 16 & Inzisalkante 21 (I21) & Mittelpunkt der Inzisalkante an 22 \\
\hline 17 & Inzisalkante 22 (I22) & Mittelpunkt der Inzisalkante an 21 \\
\hline 18 & Marginale Gingiva 12 (MG12) & Mittelpunkt der marginalen Gingiva an 12 \\
\hline 19 & Marginale Gingiva 11 (MG11) & Mittelpunkt der marginalen Gingiva an 11 \\
\hline 20 & Marginale Gingiva 21 (MG21) & Mittelpunkt der marginalen Gingiva an 21 \\
\hline 21 & Marginale Gingiva 22 (MG22) & Mittelpunkt der marginalen Gingiva an 22 \\
\hline 22 & Mesiobukkaler Höcker 36 (Mb36) & Höchster Punkt des mesiobukkalen Höckers an 36 \\
\hline 23 & Mesiobukkaler Höcker 46 (Mb 46) & Höchster Punkt des mesiobukkalen Höckers an 46 \\
\hline
\end{tabular}


Tabelle 2.4: Definition der anatomischen Linien.

\begin{tabular}{|c|c|c|}
\hline \# & Bezeichnung & Definition \\
\hline 1 & esthetic line (el) & Linie von Nasenspitze (Nspitze) zu Kinn (K) \\
\hline 2 & Zahnachse 12 (Za12) & $\begin{array}{l}\text { Linie von Inzisalkante } 12 \text { (I12) zu Marginale Gingiva } 12 \\
\text { (MG12) }\end{array}$ \\
\hline 3 & Zahnachse 11 (Za11) & $\begin{array}{l}\text { Linie von Inzisalkante } 11 \text { (I11) zu Marginale Gingiva } 11 \\
\text { (MG11) }\end{array}$ \\
\hline 4 & Zahnachse 21 (Za21) & $\begin{array}{l}\text { Linie von Inzisalkante } 21 \text { (I21) zu Marginale Gingiva } 21 \\
\text { (MG21) }\end{array}$ \\
\hline 5 & Zahnachse 22 (Za22) & $\begin{array}{l}\text { Linie von Inzisalkante } 22 \text { (I22) zu Marginale Gingiva } 22 \\
\text { (MG22) }\end{array}$ \\
\hline
\end{tabular}

Tabelle 2.5: Definition der anatomischen Ebenen.

\begin{tabular}{|c|c|c|}
\hline \# & Bezeichnung & Definition \\
\hline 1 & $\begin{array}{l}\text { Horizontalebene / natural head } \\
\text { position (NHP) }\end{array}$ & $\begin{array}{l}\text { Ebene orthogonal zur Betrachtungsachse durch } \\
\text { Exocanthion re. und li. (Ecr und Ecl) }\end{array}$ \\
\hline 2 & Axialebene $(A x)$ & $\begin{array}{l}\text { Ebene orthogonal zur Horizontalebene (NHP) durch } \\
\text { Nasion (N) und Subnasale (Sn) }\end{array}$ \\
\hline 3 & Koronalebene (Ko) & $\begin{array}{l}\text { Ebene orthogonal zur Horizontalebene (NHP) und } \\
\text { Axialebene (Ax) durch Nasion }(\mathrm{N})\end{array}$ \\
\hline 4 & $\begin{array}{l}\text { functional aesthetic occlusal plane } \\
\text { (FAOP) }\end{array}$ & $\begin{array}{l}\text { Ebene durch Mesiobukkalen Höcker } 36 \text { und } 46 \text { (Mb36 } \\
\text { und Mb46) und durch Mittelpunkt der Lippenschlusslinie } \\
\text { (ML) }\end{array}$ \\
\hline
\end{tabular}


Die functional aesthetic occlusal plane gibt Auskunft darüber, wie ästhetisch die Position des Frontzahnsegments ist. Hierzu wird der Abstand von der Inzisalkante der maxillären Frontzähne in Bezug zur functional aesthetic occlusal plane in mm gemessen. Die Inzisalkante sollte sich $2-4 \mathrm{~mm}$ unterhalb dieser Ebene befinden, um ein ästhetisches Erscheinungsbild entstehen zu lassen (Camara und Martins 2016).

Die esthetic line nach Ricketts wurde ursprünglich zur kephalometrischen Vermessung eingeführt (Ricketts 1960). In dieser Studie wird sie für die dreidimensionale Analyse verwendet, indem zwischen der Nasenspitze und dem Ende des Kinns, genauer dem Weichteilpogonion, eine Linie aufgespannt wird. Die Punkte auf der Inzisalkante des Frontzahnsegments, labium superius und labium inferius wurden zur esthetic line in Bezug gesetzt und der kürzeste Abstand in z-Achsenrichtung in mm gemessen. Des Weiteren wurde der Abstand zwischen labium superius und den Mittelpunkten der Inzisalkanten des maxillären Frontzahnsegments bestimmt. Die gleiche Messung wurde auch von labium inferius aus durchgeführt (Kuhn et al. 2016). Die Lage im Koordinatensystem von labium superius (LS), R 1/2Chp, RChp und LChp, L1/2Chp wurde zum Ursprung Nasion $(\mathrm{N})$ in allen drei Ebenen $(\mathrm{x}, \mathrm{y}, \mathrm{z})$, angelehnt an Kim et al. (2014), erfasst. Der Winkel zwischen der Zahnachse jedes maxillären Frontzahnes und der Horizontalebene / natural head position wurde zur Ermittlung des Protrusionsgrades erhoben. 

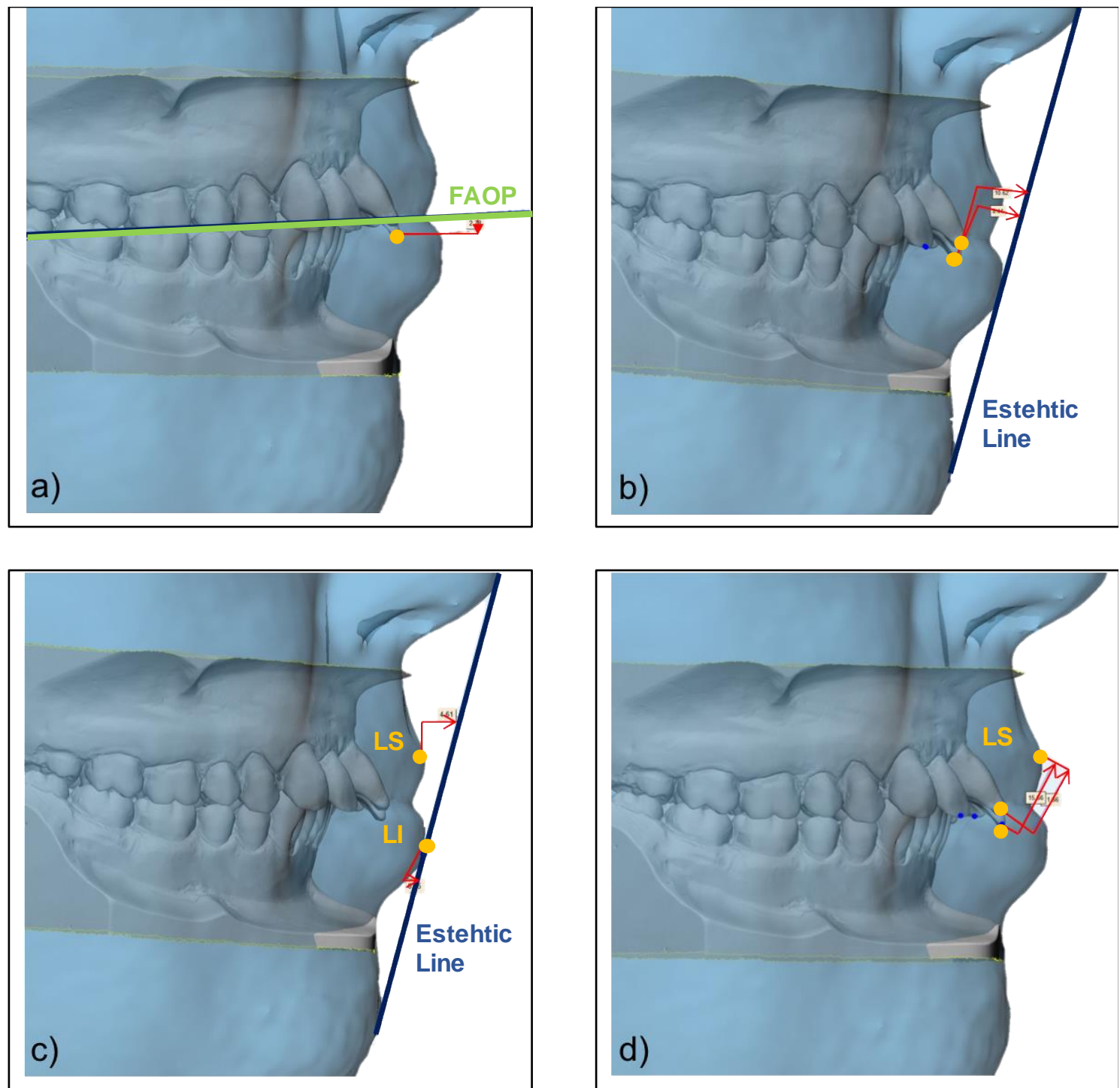

Abbildung 2.8: Messungen im dreidimensionalen Raum in dem Programm 3-matic Research 13.0. a) Inzisalkantenabstand zur FAOP, b) Abstand esthetic line zur Inzisalkante der Frontzähne, c) Abstand esthetic line zu labium superius und Abstand esthetic line zu labium inferius, d) Abstand labium superius zu Inzisalkanten der Frontzähne. FAOP: functional aesthetic occlusal plane, LS: labium superius, LI: labium inferius, NHP: natural head position. 

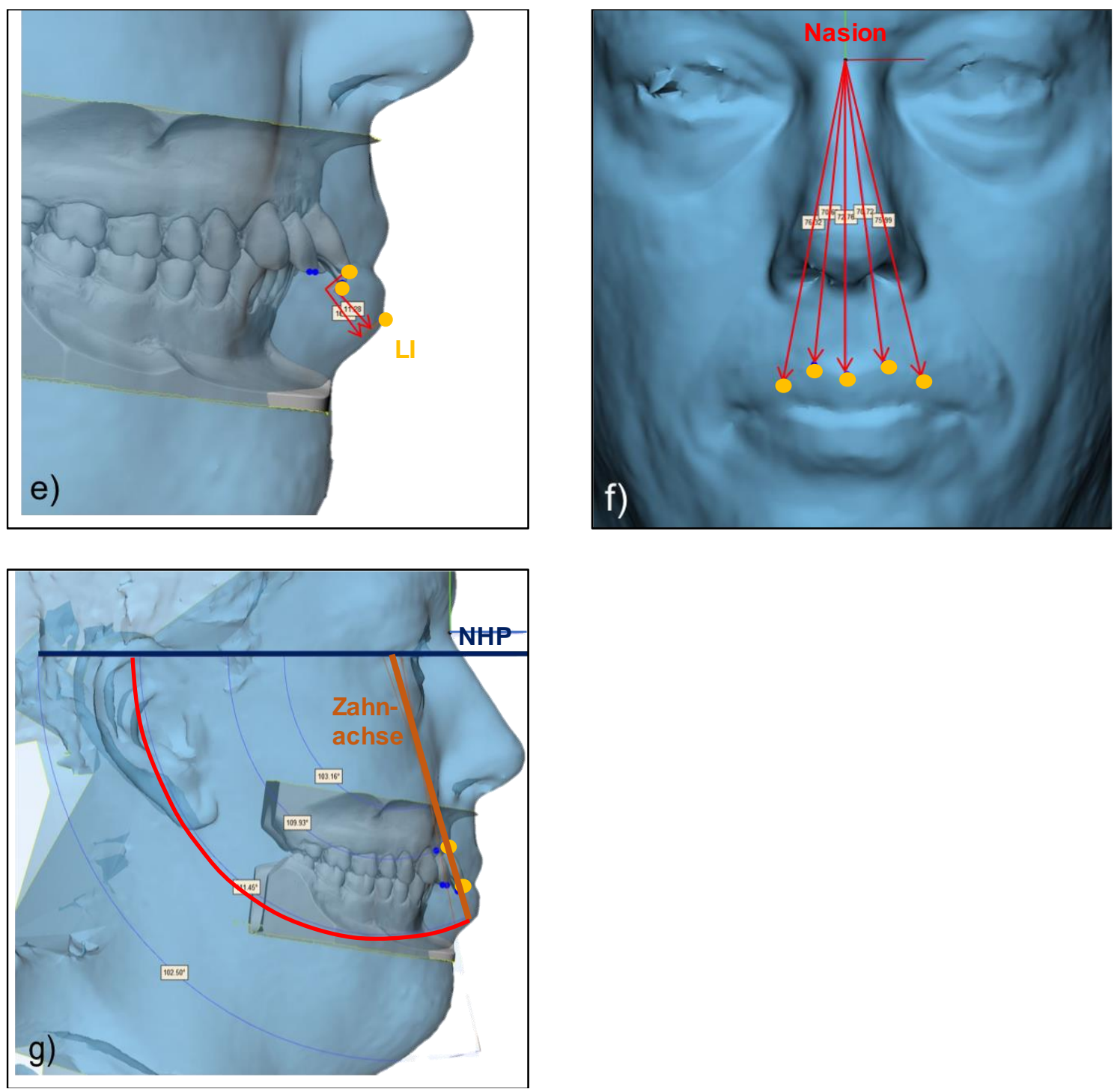

Abbildung 2.9: Messungen im dreidimensionalen Raum in dem Programm 3-matic Research 13.0. e) Abstand labium inferius zu Inzisalkanten der Frontzähne, f) labium superius (LS), R 1 1/2Chp, RChp und LChp, L1/2Chp zum Nasion, g) Winkel zwischen Zahnachse und Horizontalebene. FAOP: functional aesthetic occlusal plane, LS: labium superius, LI: labium inferius, NHP: natural head position. 


\subsection{Index of treatment need}

Der index of treatment need ist ein international etablierter Index und gibt eine Auskunft über den kieferorthopädischen Behandlungsbedarf (Brook und Shaw 1989). Der Index beinhaltet eine ästhetische Komponente (AC) und eine Komponente der dentalen Gesundheit (DHC). Die ästhetische Komponente wird im Folgenden nicht weiter behandelt.

Die Komponenten der dentalen Gesundheit werden in fünf Grade unterteilt, wobei Grad 1 für keinen Behandlungsbedarf und Grad 5 für einen definitiven Behandlungsbedarf steht. Bei der Beurteilung der Dentition wird nach einer hierarchischen Skala vorgegangen, in der immer das am stärksten ausgeprägte okklusale Merkmal beurteilt wird. Folgende Reihenfolge wird eingehalten: fehlende Zähne, overjet, Kreuzbiss, Kontaktpunktverlagerung und overbite. In diesem Fall wurden fehlende Zähne und Kreuzbiss vernachlässigt. Die verschiedenen Grade werden wie folgt beschrieben:

Tabelle 2.6: Definition des index of treatment need

\begin{tabular}{ll}
\hline index of treatment need & Definition \\
\hline Grad 1 & kein Behandlungsbedarf \\
Grad 2 & geringer Behandlungsbedarf \\
Grad 3 & grenzwertiger Behandlungsbedarf \\
Grad 4 & großer Behandlungsbedarf \\
Grad 5 & sehr großer Behandlungsbedarf \\
\hline
\end{tabular}

Jedem der fünf Grade sind durch die bestehende Literatur exakte Werte, für jede der zu betrachtenden Komponenten, zugeordnet (Brook und Shaw 1989). 


\subsection{Fragebögen}

Die verwendeten Fragebögen ermöglichten zum einen die Erhebung der mundgesundheitsbezogenen Lebensqualität jedes einzelnen Patienten (oral health impact profile und general / geriatric oral health assessment index ) und quantifizierten zum anderen einen patientenindividuellen kieferorthopädischen Behandlungswunsch (kieferorthopädischer Fragebogen).

\subsubsection{Fragebogen zum patientenindividuellen kierferorthopädischen} Behandlungswunsch

Mit dem Fragebogen zum patientenindividuellen kieferorthopädischen Behandlungswunsch soll deskriptiv das Interesse der Patienten an einer kieferorthopädischen Behandlung erfasst werden. Hierzu wurde erfragt, ob bereits eine kieferorthopädische Behandlung stattgefunden hatte oder ob über diese bereits aufgeklärt worden war. Außerdem sollten moderat und schwer erkrankte Patienten schildern, inwieweit sich ihr Bewusstsein für Zähne und Mundgesundheit seit einer eventuell durchgeführten parodontologischen Therapie geändert hat. Das Interesse an einer kieferorthopädischen Behandlung und der Grund für die Behandlung wurden erfragt. Des Weiteren werden die Erwartungen an die Behandlung erhoben, um herauszufinden, auf welche Aspekte hinsichtlich Funktion, Ästhetik und Gesundheit der Patient besonderen Wert legt. Der Fragebogen ist im Anhang beigefügt.

\subsubsection{Oral health impact profile}

Das oral health impact profile (OHIP) wurde von Slade und Spencer (1994) in Australien entworfen. Es dient der Quantifizierung der mundgesundheitsbezogenen Lebensqualität. Das OHIP-49 ist ein intensiv genutztes Instrument, bestehend aus 49 Fragen. Die Übersetzung des Fragebogens ins Deutsche fand durch John et al. (2002) statt. Da die Anzahl der Fragen des OHIP-49 für bestimmte Fragestellungen zu umfangreich ist, wurden kürzere Versionen des Fragebogens abgeleitet. Die erste Kurzversion mit 14 Fragen wurde von Slade (1997) entwickelt, diese wurde ebenfalls in die deutsche Sprache übersetzt (John et al. 2002). Die Beantwortung der Fragen erfolgt auf einer ordinalen Likert-Skala, welche folgende Abstufungen umfasst: „nie“ $=0$, 
„kaum" $=1$, „ab und $z u^{\prime \prime}=2$, „oft" $=3$ und „sehr oft" $=4$. Anschließend werden aus den Antworten Summenwerte gebildet.

\subsubsection{General / geriatric oral health assessment index}

Der aus zwölf Fragen bestehende general / geriatric oral health assessment index (GOHAl) wurde im Jahr 1990 von Atchinson und Dolan entwickelt und kann als Instrument zur Erfassung der mundgesundheitsbezogenen Lebensqualität vor allem in der älteren Bevölkerung angewendet werden (Atchison und Dolan 1990; Hassel et al. 2008). Laut Öhrn und Jönsson eignet sich der GOHAI besonders gut zur Untersuchung von Patienten, welche an Parodontitis erkrankt sind (Öhrn und Jönsson 2012). Dieser Fragebogen wurde ebenfalls in die deutsche Sprache übersetzt (Hassel et al. 2008). Jede der zwölf Fragen bezieht sich auf die persönlichen Erfahrungen der letzten drei Monate und wird anhand einer Likert-Skala ( $0=$ "nie“ bis $4=$ „sehr oft") selbstständig durch den Patienten beantwortet. Es können für die Auswertung zwei verschiedene Werte des GOHAI berechnet werden. Bei der Additivmethode (ADD-GOHAl) wurden die Antwortcodes für die zwölf Fragen summiert, nachdem die Kodierung der drei positiven Worterhebungen (Beschwerden beim Schlucken, Auftreten von Beschwerden beim Essen und Zufriedenheit mit dem Aussehen der Zähne) umgekehrt wurden (Locker et al. 2001). In der Addition reicht die Skala somit von 0 - 48. Ein hoher Wert weist auf eine Einschränkung im Bereich der mundgesundheitsbezogenen Lebensqualität hin (Locker et al. 2001). Bei der einfachen Zählmethode (SC-GOHAI) werden die Antwortmöglichkeiten „ab und zu“, „oft" und „sehr oft" ausgezählt. Die Skala reicht dementsprechend von 0 - 12, wobei 12 eine schlechte mundgesundheitsbezogene Lebensqualität anzeigt (Hassel et al. 2008). 


\subsection{Statistische Analyse}

Die Auswertung erfolgte mit Hilfe des Statistik-Programmes SPSS ${ }^{\circledR}$ Statistics (Version 25, IBM ${ }^{\circledast}$ Corp., Armonk, NY, USA). Als Signifikanzniveau wurde $\alpha=0,05$ definiert. Für die parodontal-klinischen Parameter und kieferorthopädisch-digitalen Parameter wurde eine deskriptive Statistik (Mittelwert, 95 \%-Konfidenzintervall) errechnet. Die vorliegenden Daten wurden mit Hilfe des Shapiro-Wilks-Tests auf eine Normalverteilung getestet, welche nicht vorlag.

Um die statistischen Unterschiede zwischen den Gruppen (Patienten mit schwerer Parodontitis, Patienten mit moderater Parodontitis und Patienten ohne Parodontitis) zu untersuchen, wurden Kruskal-Wallis Tests durchgeführt. Zur Analyse der Unterschiede zwischen den einzelnen Gruppen wurden MannWhitney-U-Tests als Post-hoc Tests angewandt.

Zur Untersuchung des ordinal skalierten IOTN wurden Fisher Exact Tests durchgeführt und bedingte Wahrscheinlichkeiten errechnet.

Die Daten der dreidimensionalen Messgenauigkeit und orthodontischen Parameter wurden mit Hilfe des Spearman Korrelations-Koeffizienten auf ihre Intra-Rater Reliabilität getestet.

Für die dreidimensionale Auswertung wurden wiederum statistische Unterschiede zwischen den Gruppen (Patienten mit schwerer Parodontitis, Patienten mit moderater Parodontitis und Patienten ohne Parodontitis) mit Kruskal-Wallis Tests durchgeführt. Außerdem wurde eine weitere Unterscheidung zwischen einem physiologischen overjet und einem pathologischen overjet eingeführt und statistisch mit Kruskal-Wallis Tests überprüft. 


\section{Ergebnisse}

Die folgenden Ergebnisse beschreiben zunächst das analysierte Patientenkollektiv, die klinisch parodontalen Parameter und die Ergebnisse der Modellvermessung mit dem index of treatment need. Im Anschluss wurde die Genauigkeit des virtuellen dentofazialen Patientenmodells mit der Analyse der Zahn zu Weichteilmorphologie beschrieben. Weiterhin wurden die Ergebnisse der Patientenbefragungen zum individuellen kieferorthopädischen Behandlungswunsch und zur mundgesundheitsbezogenen Lebensqualität mittels OHIP-G 14 und dem GOHAl aufgeführt.

\subsection{Patientenkollektiv}

Von insgesamt 288 ambulanten Patienten der Poliklinik für Präventive Zahnmedizin, Parodontologie und Kariologie konnten initial 126 Patienten für die Studie untersucht werden. Anschließend wurden 8 Patienten wegen qualitativ schlechter Scaninformationen oder eines zuvor nicht erkannten offenen Bisses ausgeschlossen. Letztendlich wurden 118 Patienten (51 männlich und 67 weiblich) in diese interdisziplinäre Studie eingeschlossen. Ihr mittleres Alter betrug 58,03 Jahre (Minimum 40 Jahre, Maximum 84 Jahre).

Es konnte eine relativ homogene Verteilung in drei Gruppen erreicht werden. In der Patientengruppe ohne parodontale Erkrankung konnten 39 Patienten untersucht werden (12 männliche, 27 weibliche). Das mittlere Alter betrug 57,36 Jahre (Minimum 40 Jahre, Maximum 78 Jahre). In der Patientengruppe mit moderater Parodontitis konnten ebenfalls 39 Patienten gewonnen werden (18 männliche, 21 weibliche). Das mittlere Alter betrug 60,97 Jahre (Minimum 40 Jahre, Maximum 84 Jahre). In der Patientengruppe mit schwerer Parodontitis konnten 40 Teilnehmer untersucht werden (21 männliche, 19 weibliche). Das mittlere Alter betrug 55,83 Jahre (Minimum 41 Jahre, Maximum 72 Jahre) (Tabelle 3.2). 
Eine Subklassifikation der Bisslage (Angle-Klassifikation anhand der MolarenRelation) ergab folgende Verteilung für die einzelnen Gruppen:

Tabelle 3.1: Deskriptive Statistik der Bisslage (Angle-Klassifikation) von allen Patienten bezogen auf die Gruppen „keine Parodontitis“, „moderate Parodontitis“ und „schwere Parodontitis“.

\begin{tabular}{|c|c|c|c|}
\hline & \multicolumn{3}{|c|}{$\begin{array}{l}\text { Patienten- } \\
\text { gruppen }\end{array}$} \\
\hline & $\begin{array}{c}\text { Keine } \\
\text { Parodontitis }\end{array}$ & $\begin{array}{c}\text { Moderate } \\
\text { Parodontitis }\end{array}$ & $\begin{array}{c}\text { Schwere } \\
\text { Parodontitis }\end{array}$ \\
\hline Klasse I & 28 & 27 & 25 \\
\hline Klasse II & 10 & 10 & 15 \\
\hline Klasse III & 1 & 2 & 0 \\
\hline
\end{tabular}

Tabelle 3.2: Deskriptive Statistik der Anzahl, des Alters und Geschlechts von allen Patienten bezogen auf die Gruppen „keine Parodontitis“, „moderate Parodontitis“ und „schwere Parodontitis“. Das Alter wurde als Mittelwert (MW) angegeben mit dazugehörigem Minimum (Min) und Maximum (Max). Das Alter der Patientengruppen wurde mit einem Kruskal-Wallis-Test verglichen. Der $p$-Wert wurde mit der BonferroniMethode korrigiert.

\begin{tabular}{|c|c|c|c|c|c|}
\hline & \multicolumn{3}{|c|}{$\begin{array}{l}\text { Patienten- } \\
\text { gruppen }\end{array}$} & \multirow[t]{2}{*}{$\begin{array}{l}\text { Korr. } \\
\text { p-Wert }\end{array}$} & \multirow[t]{2}{*}{$\Sigma$} \\
\hline & $\begin{array}{c}\text { Keine } \\
\text { Parodontitis }\end{array}$ & $\begin{array}{c}\text { Moderate } \\
\text { Parodontitis }\end{array}$ & $\begin{array}{c}\text { Schwere } \\
\text { Parodontitis }\end{array}$ & & \\
\hline Anzahl & 39 & 39 & 40 & - & 118 \\
\hline $\begin{array}{l}\text { Alter (MW) } \\
\text { (Min; Max) }\end{array}$ & $\begin{array}{c}57,36 \\
(40 ; 78)\end{array}$ & $\begin{array}{c}60,97 \\
(40 ; 84)\end{array}$ & $\begin{array}{c}55,83 \\
(41 ; 72)\end{array}$ & 0.071 & - \\
\hline Männlich & 12 & 18 & 21 & - & 51 \\
\hline Weiblich & 27 & 21 & 19 & - & 67 \\
\hline
\end{tabular}




\subsection{Klinisch parodontale Parameter}

\subsubsection{Parameter der Gruppeneinteilung}

Die klinisch-parodontalen Parameter der Gruppeneinteilung in Anlehnung an die Einteilung der CDC Working Group (Center for Disease Control and Prevention) der American Association of Periodontology wurden zwischen den Gruppen "keine Parodontitis", "moderate Parodontitis" und "schwere Parodontitis“ verglichen (vgl. Kapitel 2.2.1 Definition der Patientengruppen).

Die Sondierungstiefen an der Oberkieferbezahnung unterschieden sich signifikant zwischen allen drei Gruppen $(p<0,001)$. Eine stetige Zunahme der Werte konnte gezeigt werden. Im Unterkiefer waren signifikante Unterschiede zwischen Patienten der Gruppen „ohne Parodontitis“ und „schwerer Parodontitis“ $(p<0,001)$, sowie Patienten der Gruppen mit "moderater Parodontitis“ und „schwerer Parodontitis“ $(p<0,001)$ zu erkennen. Patienten mit schwerer destruktiver Parodontitis weisen nach der Einteilung der CDC Working Group demnach höhere Sondierungstiefen auf.

Des Weiteren unterschieden sich die Patienten ohne parodontale Erkrankung zu Patienten mit moderater Parodontitis $(p<0,001)$ sowie Patienten mit schwerer Parodontitis hinsichtlich der Rezessionen in Ober- und Unterkiefer signifikant $(p<0,001)$.

Der clinical attachment loss zeigte signifikante Unterschiede zwischen allen drei Gruppen $(p<0,001)$. Zusammenfassend nehmen alle klinisch-parodontalen Parameter mit der Schwere der parodontalen Destruktion zu (Tabelle 3.3). 
Tabelle 3.3: Vergleich der klinisch-parodontalen Parameter ( $\mathrm{mm}$ ) (Sondierungstiefe (ST), Rezession (RZ) und clinical attachment loss (CAL)) zwischen den Patientengruppen „keine Parodontitis“, „moderate Parodontitis“ und „schwere Parodontitis“. Die Messwerte wurden als Mittelwert (MW) mit dazugehörigem 95 \%-Konfidenzintervall $(\mathrm{Cl})$ angegeben. Die Patientengruppen wurden mit Kruskal-Wallis-Tests und Posthoc Tests verglichen. Die p-Werte wurden mit der Bonferroni-Methode korrigiert.

\begin{tabular}{|c|c|c|c|c|c|c|c|}
\hline & \multicolumn{3}{|c|}{$\begin{array}{l}\text { Patienten- } \\
\text { gruppen }\end{array}$} & \multirow[t]{2}{*}{$\begin{array}{l}\text { Korr. } \\
\text { p-Wert }\end{array}$} & \multicolumn{3}{|c|}{$\begin{array}{l}\text { Post-hoc Test } \\
\text { Korr. p-Wert }\end{array}$} \\
\hline & $\begin{array}{c}\text { Keine } \\
\text { Parodontitis } \\
\text { MW } \\
\text { (Cl } 2,5 \% \text {; } \\
\text { Cl } 97,5 \%)\end{array}$ & $\begin{array}{c}\text { Moderate } \\
\text { Parodontitis } \\
\text { MW } \\
\text { (Cl } 2,5 \% \text {; } \\
\text { Cl } 97,5 \%)\end{array}$ & $\begin{array}{c}\text { Schwere } \\
\text { Parodontitis } \\
\text { MW } \\
\text { (Cl } 2,5 \% \text {; } \\
\text { Cl } 97,5 \%)\end{array}$ & & $\begin{array}{c}\text { Keine } \\
\text { vs. } \\
\text { Moderate } \\
\text { Parodontitis }\end{array}$ & $\begin{array}{c}\text { Keine } \\
\text { vs. } \\
\text { Schwere } \\
\text { Parodontitis }\end{array}$ & $\begin{array}{c}\text { Moderate } \\
\text { vs. } \\
\text { Schwere } \\
\text { Parodontitis }\end{array}$ \\
\hline $\begin{array}{c}\text { ST } \\
\text { Maxilla }\end{array}$ & $\begin{array}{c}1,68 \\
(1,56 ; \\
1,79)\end{array}$ & $\begin{array}{c}1,91 \\
(1,77 ; \\
2,05)\end{array}$ & $\begin{array}{l}2,96 \\
(2,69 ; \\
3,22)\end{array}$ & $<0,001$ & 0,012 & $<0,001$ & $<0,001$ \\
\hline $\begin{array}{c}\text { ST } \\
\text { Mandibula }\end{array}$ & $\begin{array}{c}1,42 \\
(1,33 ; \\
1,51)\end{array}$ & $\begin{array}{c}1,58 \\
(1,45 ; \\
1,71)\end{array}$ & $\begin{array}{l}2,56 \\
(2,29 ; \\
2,83)\end{array}$ & $<0,001$ & 0,076 & $<0,001$ & $<0,001$ \\
\hline $\begin{array}{c}\mathrm{RZ} \\
\text { Maxilla }\end{array}$ & $\begin{array}{c}0,03 \\
(0,01 ; \\
0,05)\end{array}$ & $\begin{array}{c}0,30 \\
(0,18 ; \\
0,43)\end{array}$ & $\begin{array}{c}0,37 \\
(0,27 ; \\
0,46)\end{array}$ & $<0,001$ & $<0,001$ & $<0,001$ & 0,105 \\
\hline $\begin{array}{c}\mathrm{RZ} \\
\text { Mandibula }\end{array}$ & $\begin{array}{c}0,07 \\
(0,04 ; \\
0,10)\end{array}$ & $\begin{array}{l}0,55 \\
0,36 ; \\
0,73)\end{array}$ & $\begin{array}{c}0,38 \\
(0,29 ; \\
0,48)\end{array}$ & $<0,001$ & $<0,001$ & $<0,001$ & 0,533 \\
\hline $\begin{array}{c}\mathrm{CAL} \\
\text { Maxilla }\end{array}$ & $\begin{array}{c}1,71 \\
(1,59 ; \\
1,82)\end{array}$ & $\begin{array}{l}2,21 \\
(2,08 ; \\
2,34)\end{array}$ & $\begin{array}{c}3,32 \\
(3,02 ; \\
3,61)\end{array}$ & $<0,001$ & $<0,001$ & $<0,001$ & $<0,001$ \\
\hline $\begin{array}{c}\text { CAL } \\
\text { Mandibula }\end{array}$ & $\begin{array}{c}1,49 \\
(1,40 ; \\
1,58)\end{array}$ & $\begin{array}{l}2,13 \\
(1,96 ; \\
2,29)\end{array}$ & $\begin{array}{c}2,94 \\
(2,65 \\
3,24)\end{array}$ & $<0,001$ & $<0,001$ & $<0,001$ & $<0,001$ \\
\hline
\end{tabular}




\subsubsection{Lockerungsgrad}

Der Lockerungsgrad, der in vier Grade eingeteilt werden kann, stieg stetig mit Schwere der parodontalen Erkrankung. Dies zeigte sich auch in den drei verschiedenen Gruppen „ohne Parodontitis“, „moderate Parodontitis“ und „schwere Parodontitis“. Patienten mit schwerer Parodontitis wiesen deutlich öfter Lockerungen der Zähne auf. Zwischen Patienten „ohne parodontale Erkrankung“ und Patienten mit "schwerer parodontaler Erkrankung" und "moderater Parodontitis" sowie Patienten mit "moderater Erkrankung“ und "schwerer Erkrankung" ließen sich signifikante Unterschiede aufdecken $(p=0,001$, $p<0,001$ ) (Tabelle 3.4). Patienten mit schwerer parodontaler Destruktion zeigten klinisch vermehrt gelockerte Zähne, welche potentiell zu Dysfunktionen und Zahnmigrationen führen können.

Tabelle 3.4: Vergleich des Lockerungsgrades zwischen den Patientengruppen „keine Parodontitis“, „moderate Parodontitis“ und „schwere Parodontitis“. Die Lockerungsgrade wurden als Mittelwert (MW) mit dazugehörigem 95 \%-Konfidenzintervall $(\mathrm{Cl})$ angegeben. Die Patientengruppen wurden mit Kruskal-WallisTest und Post-hoc Tests verglichen. Die p-Werte wurden mit der Bonferroni-Methode korrigiert.

\begin{tabular}{|c|c|c|c|c|c|c|c|}
\hline & \multicolumn{3}{|c|}{$\begin{array}{l}\text { Patienten- } \\
\text { gruppen }\end{array}$} & \multirow[t]{2}{*}{$\begin{array}{l}\text { Korr. } \\
\text { p-Wert }\end{array}$} & \multicolumn{3}{|c|}{$\begin{array}{l}\text { Post-hoc Test } \\
\text { Korr. p-Wert }\end{array}$} \\
\hline & $\begin{array}{c}\text { Keine } \\
\text { Parodontitis } \\
\text { MW } \\
(\mathrm{Cl} 2,5 \% \text {; } \\
\mathrm{Cl} 97,5 \%) \\
\end{array}$ & $\begin{array}{c}\text { Moderate } \\
\text { Parodontitis } \\
\text { MW } \\
(\mathrm{Cl} 2,5 \% \\
\mathrm{Cl} 97,5 \%) \\
\end{array}$ & $\begin{array}{c}\text { Schwere } \\
\text { Parodontitis } \\
\text { MW } \\
\text { (Cl 2,5\%; } \\
\mathrm{Cl} \mathrm{97,5 \% )} \\
\end{array}$ & & $\begin{array}{c}\text { Keine } \\
\text { vs. } \\
\text { Moderate } \\
\text { Parodontitis }\end{array}$ & $\begin{array}{l}\text { Keine } \\
\text { vs. } \\
\text { Schwere } \\
\text { Parodontitis }\end{array}$ & $\begin{array}{c}\text { Moderate } \\
\text { vs. } \\
\text { Schwere } \\
\text { Parodontitis }\end{array}$ \\
\hline $\begin{array}{l}\text { Lockerungs- } \\
\text { grad }\end{array}$ & $\begin{array}{c}0,00 \\
(0,00 ; \\
0,00)\end{array}$ & $\begin{array}{c}0,06 \\
(0,01 \\
0,10)\end{array}$ & $\begin{array}{c}0,29 \\
(0,19 \\
0,39)\end{array}$ & $<0,001$ & 0,001 & $<0,001$ & $<0,001$ \\
\hline
\end{tabular}




\subsection{Modellvermessung - orthodontische Parameter}

Die orthodontischen Parameter wurden anhand der Gipsmodelle mit Hilfe eines millimeter-skalierten Messschiebers mit Nonius Ablesung gemessen.

Die Genauigkeit der Messungen wurde mit Hilfe des Intra-RaterReliabilitätskoeffizienten bestimmt. Dazu wurde der Spearman KorrelationsKoeffizient berechnet. Es konnte gezeigt werden, dass die Messungen sehr exakt sind (Tabelle 3.5).

Tabelle 3.5: Vergleich der Messgenauigkeit der orthodontischen Parameter zu unterschiedlichen Zeitpunkten. Für die Messungen wurde der Spearman Korrelations-Koeffizient bestimmt. Die p-Werte wurden mit der Bonferroni-Methode korrigiert.

\begin{tabular}{ccc}
\hline Procedere & $\begin{array}{c}\text { Spearman } \\
\text { Korrelations-Koeffizient }\end{array}$ & Korr.p-Wert \\
\hline Overjet & 0,912 & $<0,001$ \\
\hline Overbite & 0,991 & $<0,001$ \\
\hline $\begin{array}{c}\text { Little-Index } \\
\text { OK }\end{array}$ & 0,969 & $<0,001$ \\
\hline $\begin{array}{c}\text { Little-Index } \\
\text { UK }\end{array}$ & 0,997 & $<0,001$ \\
\hline
\end{tabular}

\subsubsection{Overjet, Overbite, Little-Index}

Der overjet war bei Patienten mit moderater parodontaler Destruktion um $30 \%$ größer als bei Patienten mit gesundem Parodont. Im Vergleich zu Patienten mit schwerer parodontaler Destruktion lag dieser Unterschied bei $69 \%$. Zwischen Patienten mit moderater Parodontitis und schwerer Parodontitis ließen sich im Vergleich zu Patienten ohne Parodontitis signifikante Unterschiede feststellen $(p=0,026, p<0,001)$.

Auch der Little-Index nahm mit steigender parodontaler Destruktion stetig zu, was auf eine ausgeprägte Malokklusion schließen lässt. Im Oberkiefer konnten signifikante Unterschiede zwischen Patienten mit gesundem Parodont und Patienten mit schwerer parodontaler Destruktion aufgedeckt werden $(p<0,001)$. Im Unterkiefer war die Irregularität bei Patienten mit schwerer parodontaler 
Destruktion signifikant höher als bei Patienten ohne parodontale Destruktion $(p=0,002)$.

Der overbite veränderte sich nicht signifikant zwischen den verschiedenen Erkrankungsgraden (Tabelle 3.6).

Tabelle 3.6: Vergleich der orthodontischen Parameter $(\mathrm{mm})$ zwischen den Patientengruppen „keine Parodontitis“, „moderate Parodontitis“ und „schwere Parodontitis“. Die Messwerte wurden als Mittelwert (MW) mit dazugehörigem 95\%-Konfidenzintervall (Cl) angegeben. Die Patientengruppen wurden mit Kruskal-Wallis-Tests und Post-hoc Tests verglichen. Die p-Werte wurden mit der Bonferroni-Methode korrigiert.

\begin{tabular}{|c|c|c|c|c|c|c|c|}
\hline & \multicolumn{3}{|c|}{$\begin{array}{l}\text { Patienten- } \\
\text { gruppen }\end{array}$} & \multirow[t]{2}{*}{$\begin{array}{l}\text { Korr. } \\
\text { p-Wert }\end{array}$} & \multicolumn{3}{|c|}{$\begin{array}{c}\text { Post-hoc Test } \\
\text { Korr. p-Wert }\end{array}$} \\
\hline & $\begin{array}{c}\text { Keine } \\
\text { Parodontitis } \\
\text { MW } \\
\text { (Cl } 2,5 \% ; \\
\text { Cl } 97,5 \%) \\
\end{array}$ & $\begin{array}{c}\text { Moderate } \\
\text { Parodontitis } \\
\text { MW } \\
\text { (Cl } 2,5 \% \text {; } \\
\text { Cl } 97,5 \%) \\
\end{array}$ & $\begin{array}{c}\text { Schwere } \\
\text { Parodontitis } \\
\text { MW } \\
\text { (Cl } 2,5 \% \\
\mathrm{Cl} 97,5 \%) \\
\end{array}$ & & $\begin{array}{c}\text { Keine } \\
\text { vs. } \\
\text { Moderate } \\
\text { Parodontitis }\end{array}$ & $\begin{array}{c}\text { Keine } \\
\text { vs. } \\
\text { Schwere } \\
\text { Parodontitis }\end{array}$ & $\begin{array}{c}\text { Moderate } \\
\text { vs. } \\
\text { Schwere } \\
\text { Parodontitis }\end{array}$ \\
\hline Overjet & $\begin{array}{c}2,60 \\
(2,10 ; \\
3,08)\end{array}$ & $\begin{array}{c}3,37 \\
(2,73 \\
4,02)\end{array}$ & $\begin{array}{c}4,38 \\
(3,58 \\
5,17)\end{array}$ & $<0,001$ & 0,026 & $<0,001$ & 0,051 \\
\hline Overbite & $\begin{array}{l}2,95 \\
(2,33 \\
3,56)\end{array}$ & $\begin{array}{l}3,10 \\
(2,68 \\
3,52)\end{array}$ & $\begin{array}{l}3,45 \\
(2,81 \\
4,09)\end{array}$ & 0,475 & - & - & - \\
\hline $\begin{array}{c}\text { Little-Index } \\
\text { OK }\end{array}$ & $\begin{array}{c}1,76 \\
(1,22 ; \\
2,30)\end{array}$ & $\begin{array}{l}2,56 \\
(1,77 \\
3,36)\end{array}$ & $\begin{array}{c}4,06 \\
(2,96 ; \\
5,16)\end{array}$ & 0,001 & 0,186 & $<0,001$ & 0,024 \\
\hline $\begin{array}{l}\text { Little-Index } \\
\text { UK }\end{array}$ & $\begin{array}{l}2,71 \\
(1,97 \\
3,44)\end{array}$ & $\begin{array}{c}3,78 \\
(2,85 \\
4,72)\end{array}$ & $\begin{array}{c}4,33 \\
(3,46 \\
5,19)\end{array}$ & 0,012 & 0,098 & 0,002 & 0,395 \\
\hline
\end{tabular}




\subsection{Dentofaziales Modell}

\subsubsection{Virtuelles Patientenmodell - Überlagerungspräzision}

Die Genauigkeit der Triangulation (Matching) aller Datensätze wurde mit Hilfe des Intra-Rater-Reliabilitätskoeffizienten bestimmt. Die Scatter Plots beider Registrierungsdurchgänge zeigten, dass die Varianzen überall gleich waren und kein Trend zu erkennen war. (Abbildung 3.1, Abbildung 3.2). Die Messungen der Überlagerungen zu unterschiedlichen Zeitpunkten von einer durchführenden Person wurden mit dem Spearman Korrelations-Koeffizient auf ihre Übereinstimmung getestet. Hohe Werte, gegen eins gehend, weisen hierbei auf sehr gute Vorhersagewerte, beziehungsweise Übereinstimmungen von erster zu zweiter Messung hin. Die Überlagerung von Gipsmodell mit dem Weichteilscan mit Wangenabhaltern (Registrierung I) zeigte einen Spearman KorrelationsKoeffizient von 0,896 $(p<0,001)$. Bei der Überlagerung von Weichteilscan mit Wangenabhaltern und Weichteilscan in Okklusion (Registrierung II) zeigte sich ein Spearman Korrelations-Koeffizient von $0,724 \quad(p<0,001)$. Beide Messergebnisse spiegeln eine hohe Übereinstimmung wider, mit klinisch irrelevanter Varianz (Tabelle 3.7). 


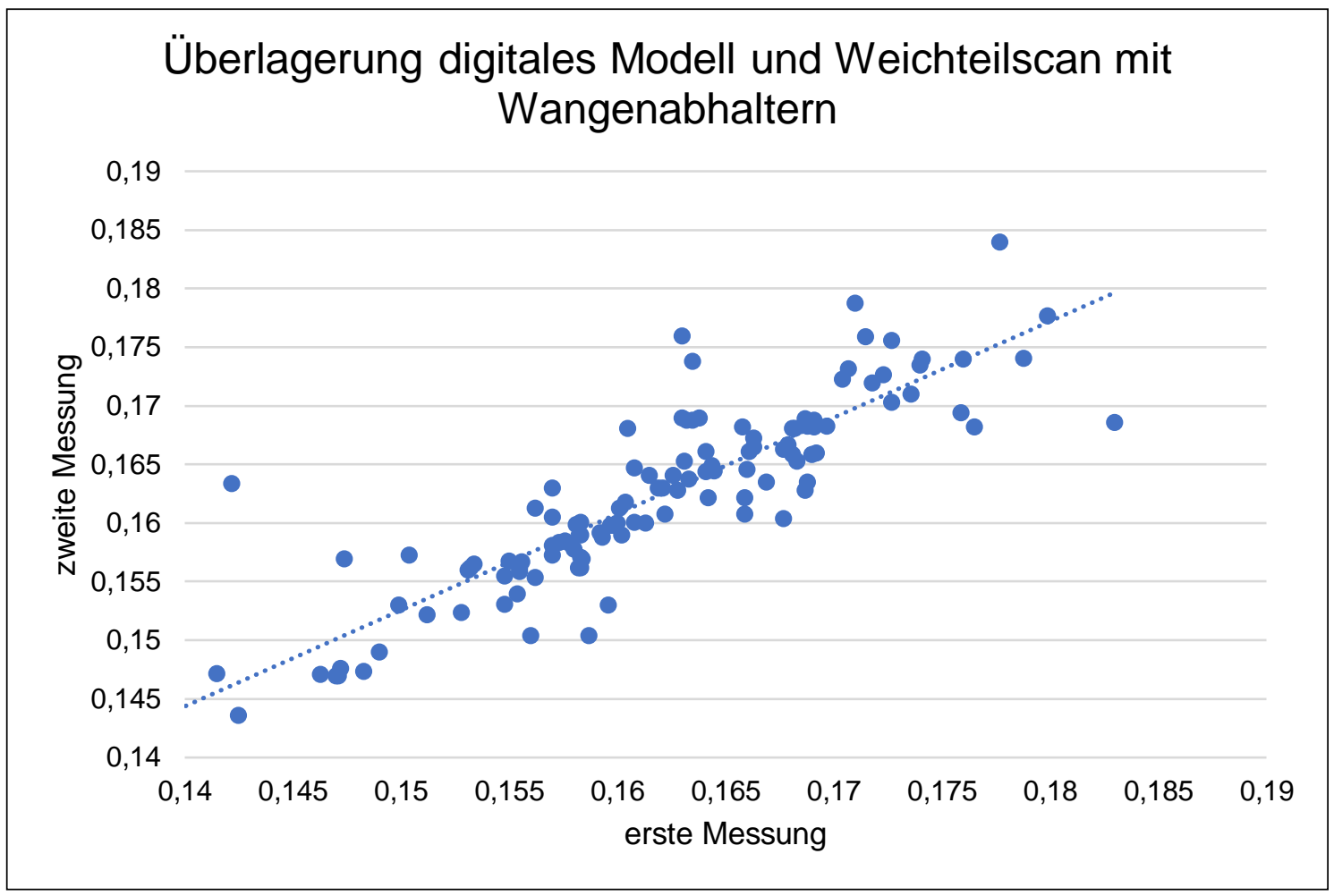

Abbildung 3.1: Scatter Plot Registrierung I. Beobachtete Wertepaare der Überlagerung von digitalem Modell und Weichteilscan mit Wangenabhaltern. Trendlinie zur Veranschaulichung der Daten, Einheit in mm.

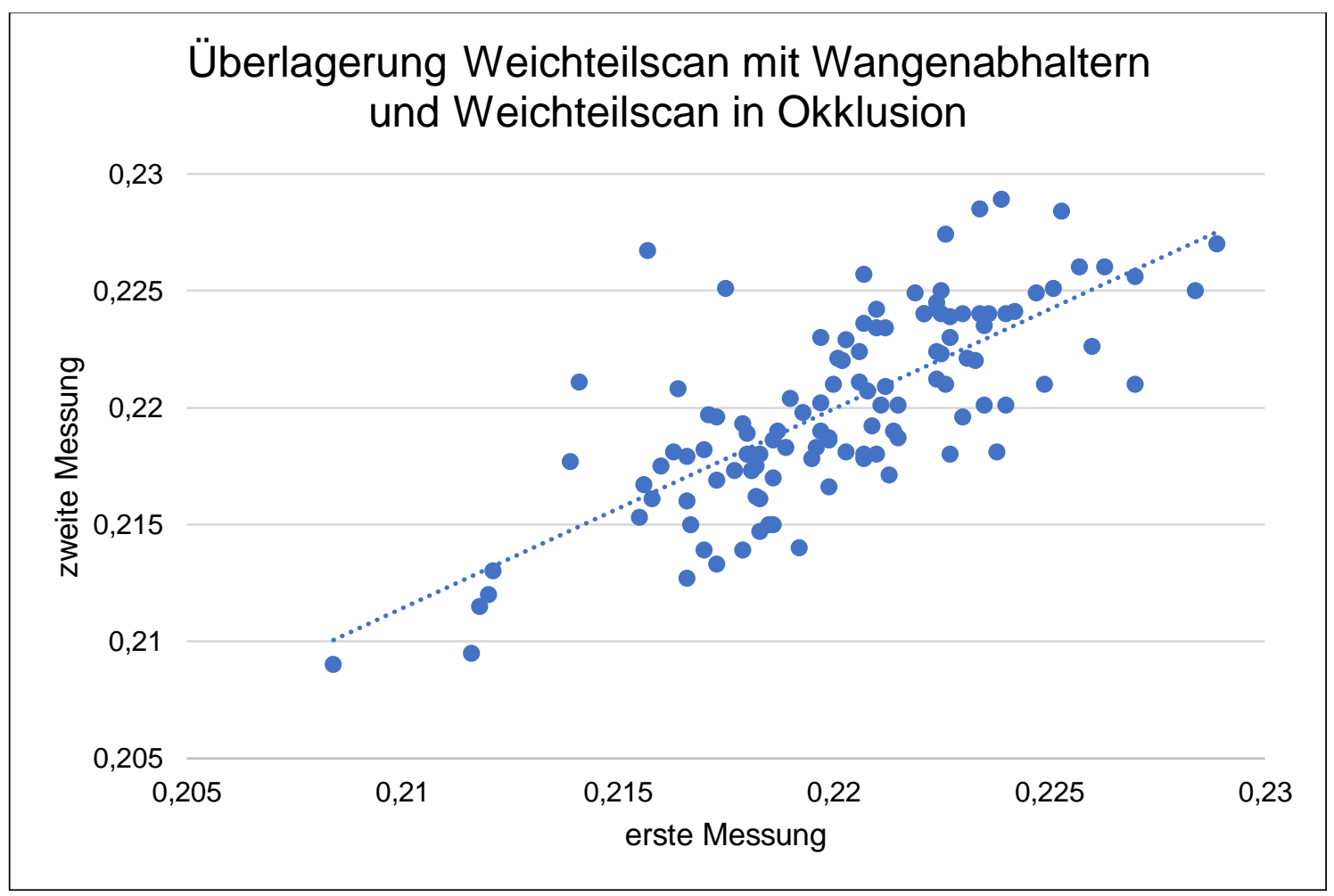

Abbildung 3.2: Scatter Plot Registrierung II. Beobachtete Wertepaare der Überlagerung von Weichteilscan in Okklusion und Weichteilscan mit Wangenabhaltern. Trendlinie zur Veranschaulichung der Daten, Einheit in $\mathrm{mm}$. 
Tabelle 3.7: Vergleich der Überlagerungspräzision $(\mathrm{mm})$ zu unterschiedlichen Zeitpunkten. Die Differenzen zwischen den verschiedenen Objekten wurden in Mittelwerten (MW) angegeben mit dazugehörigem 95 \%Konfidenzintervall (Cl). Für die Messungen (Messung 1 (M1) und Messung 2 (M2)) wurden die Spearman Korrelationen bestimmt. Die $\mathrm{p}$-Werte wurden mit der Bonferroni-Methode korrigiert.

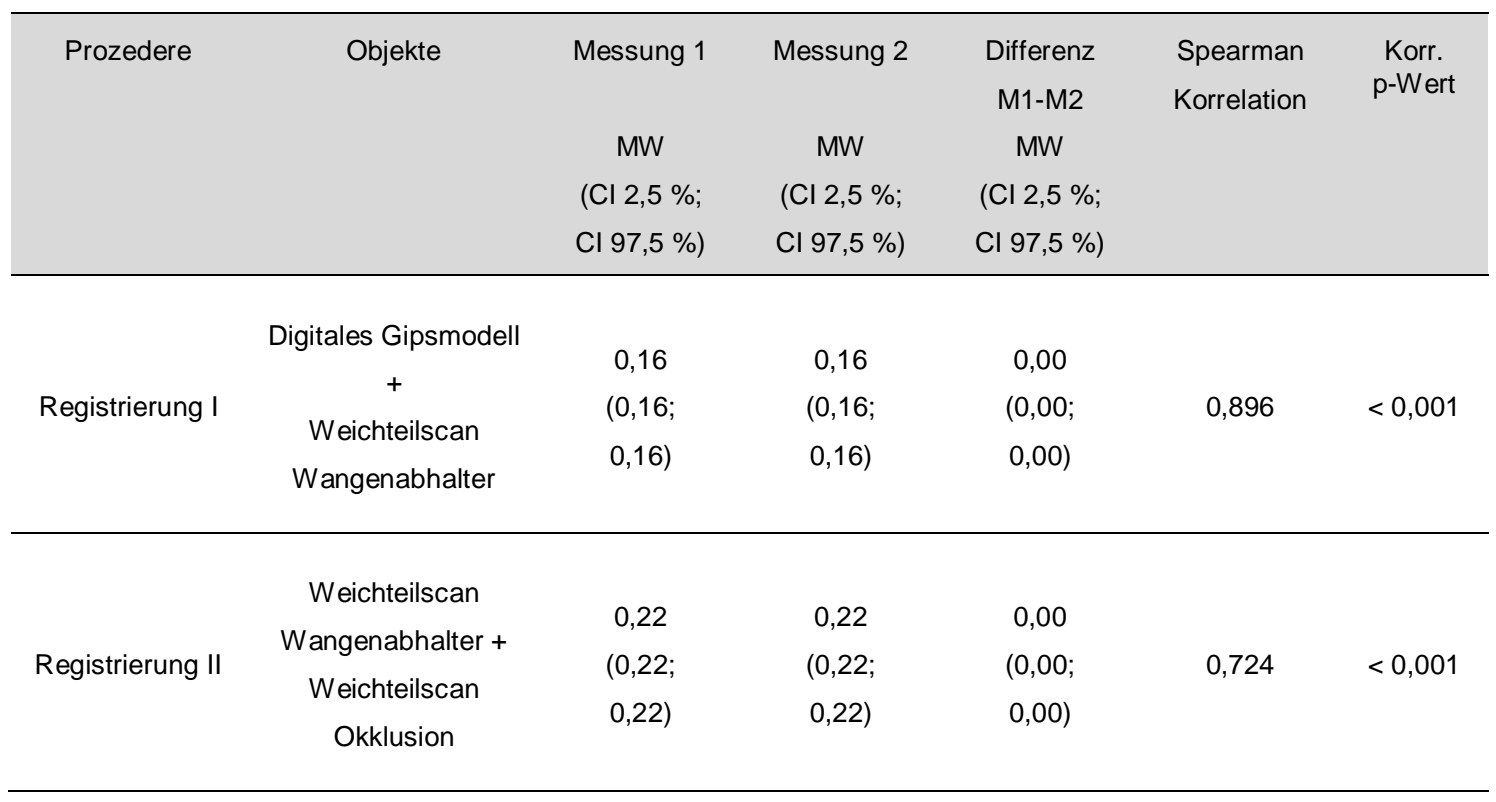




\subsubsection{Ergebnisse der dreidimensionalen Analyse der}

Frontzahn - Weichteilrelation

Die Auswertung der dreidimensionalen Patientenmodelle zeigte keine Unterschiede bezogen auf den Schweregrad der parodontalen Erkrankung. Der Winkel zwischen der Zahnachse und der natural head position unterschied sich nicht für Patienten ohne parodontale Erkrankung, Patienten mit moderater parodontaler Erkrankung und schwerer parodontaler Erkrankung. Der Abstand der Inzisalkante zur functional aesthetic occlusal plane war nicht signifikant unterschiedlich zwischen den Gruppen. Von der esthetic line zur Inzisalkante der Zähne und zu labium superius und labium inferius war zwischen den verschiedenen Erkrankungsgraden kein signifikanter Unterschied auszumachen (Tabelle 3.8).

Tabelle 3.8: Vergleich der dreidimensionalen Parameter der Patientengruppen „keine Parodontitis“, „moderate Parodontitis“ und „schwere Parodontitis“. Die Messwerte wurden als Mittelwert (MW) mit dazugehörigem 95 \%-Konfidenzintervall (Cl) angegeben. Die Patientengruppen wurden mit Kruskal-WallisTests verglichen. Die p-Werte wurden mit der Bonferroni-Methode korrigiert.

\begin{tabular}{|c|c|c|c|c|}
\hline & \multicolumn{3}{|c|}{$\begin{array}{c}\text { Patienten- } \\
\text { gruppen }\end{array}$} & \multirow[t]{2}{*}{$\begin{array}{l}\text { Korr. } \\
\text { p-Wert }\end{array}$} \\
\hline & $\begin{array}{c}\text { Keine Parodontitis } \\
\text { MW } \\
\text { (Cl } 2,5 \% \\
\text { Cl } 97,5 \%)\end{array}$ & $\begin{array}{l}\text { Moderate Parodontitis } \\
\text { MW } \\
\text { (Cl } 2,5 \% \\
\text { Cl } 97,5 \%)\end{array}$ & $\begin{array}{c}\text { Schwere Parodontitis } \\
\text { MW } \\
(\mathrm{Cl} 2,5 \% \\
\mathrm{Cl} 97,5 \%)\end{array}$ & \\
\hline $\begin{array}{l}\text { Winkel zwischen } \\
\text { Zahnachsen und } \\
\text { natural head } \\
\text { position }\left(^{\circ}\right)\end{array}$ & $\begin{array}{c}90,76 \\
(87,58 \\
93,93)\end{array}$ & $\begin{array}{c}93,39 \\
(90,14 \\
96,63)\end{array}$ & $\begin{array}{c}91,14 \\
(87,72 ; \\
94,55)\end{array}$ & 0,361 \\
\hline $\begin{array}{c}\text { Überragen der } \\
\text { Frontzähne über die } \\
\text { functional aesthetic } \\
\text { occlusal plane (mm) }\end{array}$ & $\begin{array}{c}0,45 \\
(-0,24 \\
1,14)\end{array}$ & $\begin{array}{c}0,47 \\
(-0,26 \\
1,19)\end{array}$ & $\begin{array}{c}1,27 \\
(0,55 \\
1,98)\end{array}$ & 0,186 \\
\hline $\begin{array}{l}\text { Abstand esthetic } \\
\text { line zu Zähnen } \\
(\mathrm{mm})\end{array}$ & $\begin{array}{c}16,18 \\
(14,74 ; \\
17,59)\end{array}$ & $\begin{array}{c}16,31 \\
(15,17 \\
17,45)\end{array}$ & $\begin{array}{c}16,53 \\
(15,19 ; \\
17,87)\end{array}$ & 0,587 \\
\hline $\begin{array}{l}\text { Abstand esthetic } \\
\text { line zu labium } \\
\text { inferius }(\mathrm{mm})\end{array}$ & $\begin{array}{c}5,62 \\
(4,83 \\
6,41)\end{array}$ & $\begin{array}{c}5,78 \\
(4,91 \\
6,65)\end{array}$ & $\begin{array}{c}5,08 \\
(4,04 \\
6,12)\end{array}$ & 0,415 \\
\hline $\begin{array}{l}\text { Abstand esthetic } \\
\text { line zu labium } \\
\text { superius (mm) }\end{array}$ & $\begin{array}{l}7,08 \\
(6,22 \\
7,93)\end{array}$ & $\begin{array}{l}6,95 \\
(5,95 \\
7,96)\end{array}$ & $\begin{array}{c}6,63 \\
(5,70 ; \\
7,55)\end{array}$ & 0,637 \\
\hline
\end{tabular}


Die Einteilung des overjets in einen physiologischen Bereich bis $+2 \mathrm{~mm}$ und einen pathologischen Bereich von $>+2 \mathrm{~mm}$ wurde für eine weiterführende Auswertung angewandt. Es zeigten sich keine signifikanten Unterschiede für den Winkel zwischen Zahnachse und natural head position und den Abstand der functional aesthetic occlusal plane zur Inzisalkante der Zähne. Beim Abstand von der esthetic line zu labium inferius waren zwischen dem physiologischen und pathologischen overjet signifikante Unterschiede $(p=0,006)$ zu beobachten (Tabelle 3.9).

Tabelle 3.9: Vergleich der dreidimensionalen Parameter zwischen physiologischem und pathologischem overjet. Die Messwerte wurden als Mittelwert (MW) mit dazugehörigem $95 \%$ Konfidenzintervall $(\mathrm{Cl})$ angegeben. Die Gruppen wurden mit Kruskal-Wallis-Tests verglichen. Die p-Werte wurden mit der Bonferroni-Methode korrigiert

\begin{tabular}{|c|c|c|c|}
\hline & \multicolumn{2}{|c|}{$\begin{array}{l}\text { Patienten- } \\
\text { gruppen }\end{array}$} & \multirow[t]{2}{*}{$\begin{array}{l}\text { Korr. } \\
p-\text { Wert }\end{array}$} \\
\hline & $\begin{array}{l}\text { Physiologischer overjet } \\
\text { MW } \\
\text { (Cl } 2,5 \% \\
\text { Cl } 97,5 \%)\end{array}$ & $\begin{array}{c}\text { Pathologischer overjet } \\
\text { MW } \\
(\mathrm{Cl} 2,5 \% \\
\mathrm{Cl} 97,5 \%)\end{array}$ & \\
\hline $\begin{array}{c}\text { Winkel zwischen } \\
\text { Zahnachsen und natural } \\
\text { head position }\left(^{\circ}\right)\end{array}$ & $\begin{array}{c}89,51 \\
(86,23 \\
92,79)\end{array}$ & $\begin{array}{l}93,04 \\
(90,81 \\
95,27)\end{array}$ & 0,093 \\
\hline $\begin{array}{c}\text { Überragen der Frontzähne } \\
\text { über die functional } \\
\text { aesthetic occlusal plane } \\
(\mathrm{mm})\end{array}$ & $\begin{array}{c}0,33 \\
(-0,21 \\
0,88)\end{array}$ & $\begin{array}{c}0,96 \\
(0,41 \\
1,52)\end{array}$ & 0,356 \\
\hline $\begin{array}{c}\text { Abstand esthetic line zu } \\
\text { Zähnen }(\mathrm{mm})\end{array}$ & $\begin{array}{c}16,95 \\
(15,99 \\
17,90)\end{array}$ & $\begin{array}{c}15,98 \\
(14,96 \\
17,00)\end{array}$ & 0,254 \\
\hline $\begin{array}{l}\text { Abstand esthetic line zu } \\
\text { labium inferius }(\mathrm{mm})\end{array}$ & $\begin{array}{l}5,89 \\
(5,14 \\
6,65)\end{array}$ & $\begin{array}{l}5,26 \\
(4,56 \\
5,95)\end{array}$ & 0,006 \\
\hline $\begin{array}{l}\text { Abstand esthetic line zu } \\
\text { labium superius (mm) }\end{array}$ & $\begin{array}{l}7,72 \\
(6,98 \\
8,45)\end{array}$ & $\begin{array}{l}6,40 \\
(5,70 \\
7,09)\end{array}$ & 0,157 \\
\hline
\end{tabular}




\subsection{Index of treatment need}

Der index of treatment need wurde hinsichtlich der dentalen Gesundheit zwischen den Patientengruppen "ohne Parodontitis“, „moderater Parodontitis“ und "schwerer Parodontitis“ verglichen. Es wurden fünf IOTN-Level erhoben. Ein hohes Level beschreibt hierbei einen erhöhten Behandlungsbedarf. Ein stetiger Anstieg von einem grenzwertigen Behandlungsbedarf auf einen großen beziehungsweise sehr großen Behandlungsbedarf konnte beobachtet werden. Patienten mit schwerer Parodontitis wiesen signifikant höhere IOTN-Level auf als Patienten ohne diese Erkrankung $(p=0,005)$. Die IOTN-Level zwischen den anderen Patientengruppen unterschieden sich nicht signifikant. Schwer Erkrankte wurden zu $50 \%$ in die Kategorie des großen beziehungsweise sehr großen Behandlungsbedarfs eingestuft. Hingegen wurden Patienten ohne parodontale Erkrankung zu über $40 \%$ einem geringen Behandlungsbedarf zugeordnet. Diese Patienten zeigten geringe bis grenzwertige Behandlungsbedürftigkeiten. Bei moderat erkrankten Patienten war ein grenzwertiger bis großer Behandlungsbedarf festzustellen. Ein großer bis sehr großer Behandlungsbedarf konnte bei Patienten mit schwerer parodontaler Erkrankung erhoben werden (Abbildung 3.3, Tabelle 3.10). 


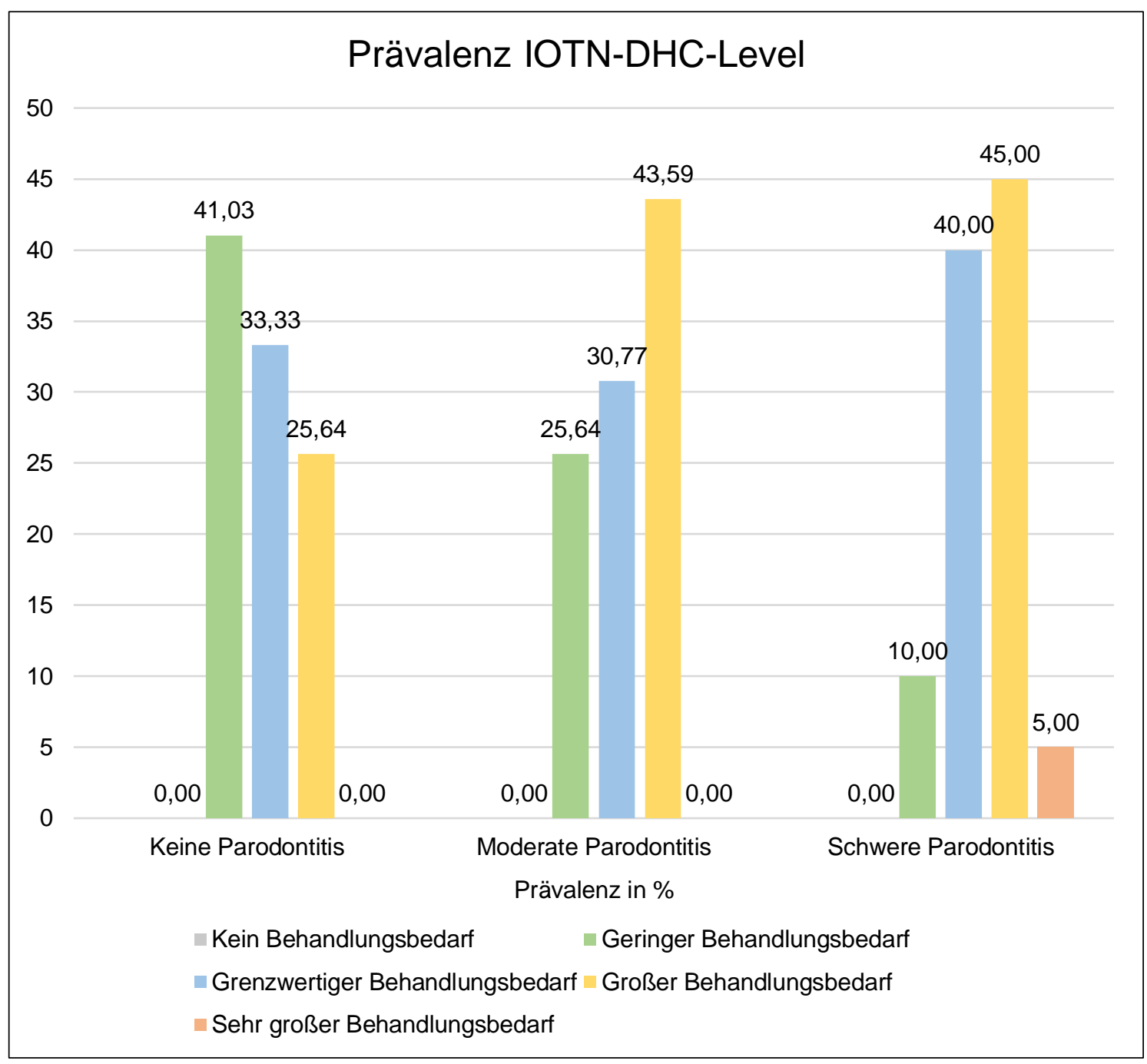

Abbildung 3.3: Prävalenz der IOTN-DHC-Level bezogen auf die Patientengruppen „Keine Parodontitis“, „Moderate Parodontitis“, „Schwere Parodontitis“.

Tabelle 3.10: Vergleich der IOTN-DHC Level zwischen den Patientengruppen „keine Parodontitis“, „moderate Parodontitis“ und „schwere Parodontitis“. Die Level wurden als Mittelwert (MW) mit dazugehörigem 95 \%-Konfidenzintervall $(\mathrm{Cl})$ angegeben. Die Patientengruppen wurden mit Fisher Exact Tests und Post-hoc Tests verglichen. Die p-Werte wurden mit der Bonferroni-Methode korrigiert.

\begin{tabular}{|c|c|c|c|c|c|c|c|}
\hline & \multicolumn{3}{|c|}{$\begin{array}{l}\text { Patienten- } \\
\text { gruppen }\end{array}$} & \multirow[t]{2}{*}{$\begin{array}{l}\text { Korr. } \\
p-\text { Wert }\end{array}$} & \multicolumn{3}{|c|}{$\begin{array}{c}\text { Post-hoc Test } \\
\text { Korr. p-Wert }\end{array}$} \\
\hline & $\begin{array}{c}\text { Keine } \\
\text { Parodontitis } \\
\text { MW } \\
\text { (Cl } 2,5 \% \text {; } \\
\text { Cl } 97,5 \%) \\
\end{array}$ & $\begin{array}{c}\text { Moderate } \\
\text { Parodontitis } \\
\text { MW } \\
\text { (Cl } 2,5 \% \\
\text { Cl } 97,5 \%) \\
\end{array}$ & $\begin{array}{c}\text { Schwere } \\
\text { Parodontitis } \\
\text { MW } \\
\text { (Cl } 2,5 \% \text {; } \\
\text { Cl } 97,5 \%) \\
\end{array}$ & & $\begin{array}{c}\text { Keine } \\
\text { vs. } \\
\text { Moderate } \\
\text { Parodontitis }\end{array}$ & $\begin{array}{c}\text { Keine } \\
\text { vs. } \\
\text { Schwere } \\
\text { Parodontitis }\end{array}$ & $\begin{array}{c}\text { Moderate } \\
\text { vs. } \\
\text { Schwere } \\
\text { Parodontitis }\end{array}$ \\
\hline $\begin{array}{l}\text { IOTN- } \\
\text { DHC- } \\
\text { Level }\end{array}$ & $\begin{array}{c}2,85 \\
(2,58 ; \\
3,11)\end{array}$ & $\begin{array}{c}3,18 \\
(2,91 ; \\
3,45)\end{array}$ & $\begin{array}{c}3,45 \\
(3,21 ; \\
3,69)\end{array}$ & 0,022 & 0,205 & 0,005 & 0,165 \\
\hline
\end{tabular}




\subsection{Fragebögen}

3.6.1 Ergebnisse des patientenindividuellen kieferorthopädischen Fragebogens

Der patientenindividuelle kieferorthopädische Fragebogen quantifiziert das Bewusstsein für Mundgesundheit, Interesse und Erwartungen hinsichtlich einer kieferorthopädischen Therapie und stellt Informationsmöglichkeiten dar.

\subsubsection{Bewusstseinsveränderung hinsichtlich der Mundgesundheit seit der} parodontologischen Therapie

Die Patienten mit moderater oder schwerer parodontaler Destruktion wurden befragt, ob sich ihr Bewusstsein für Zähne, Mund und Mundgesundheit seit der parodontalen Behandlung verändert hat. Es fand keine Unterscheidung zwischen den moderat und schwer erkrankten Patienten statt. $39 \%$ der Befragten gaben an, dass sich ihr Bewusstsein sehr deutlich verändert habe. Wiederum $43 \%$ berichteten von einer deutlichen Veränderung und nur insgesamt $18 \%$ nahmen geringe bis gar keine Veränderung wahr (Abbildung 3.4).

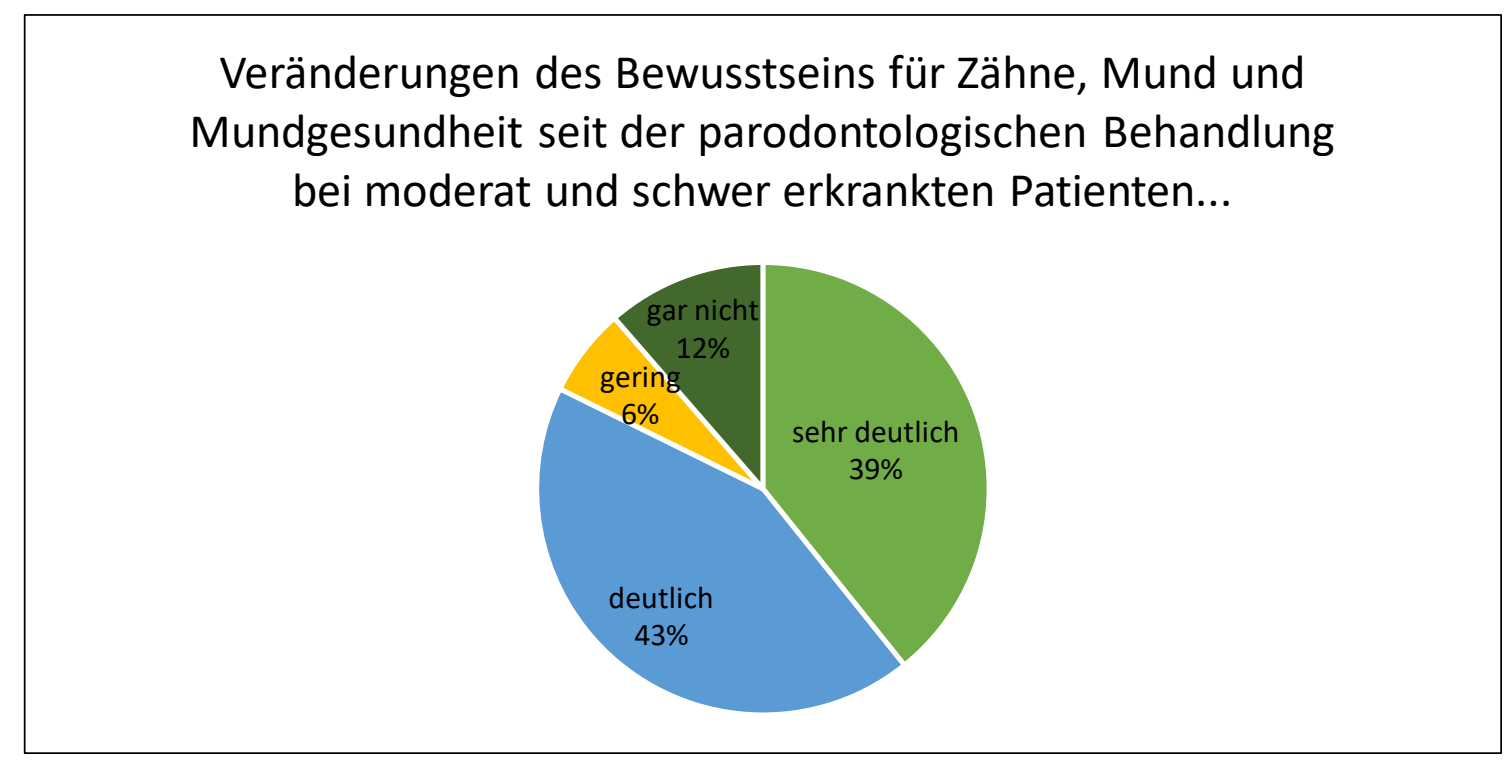

Abbildung 3.4: Kreisdiagramm zur Verteilung der Veränderung des Bewusstseins für Zähne, Mund und Mundgesundheit seit der parodontologischen Behandlung bei moderat und schwer erkrankten Patienten. 


\subsubsection{Informationen über eine kieferorthopädische Therapie}

Alle Patienten wurden befragt, ob und durch wen sie in der Vergangenheit über kieferorthopädische Behandlungsmöglichkeiten informiert wurden. Mehrfachantworten waren möglich. Es fand keine Unterscheidung zwischen den Patientengruppen statt. $44 \%$ der Befragten gaben an, noch nie über die Möglichkeit einer kieferorthopädischen Behandlung aufgeklärt worden zu sein. Durch den Zahnarzt wurden 36 \% informiert. Der Kieferorthopäde selbst hat $11 \%$ der Patienten über seine Behandlungsmöglichkeiten in Kenntnis gesetzt. Jeweils $3 \%$ der Patienten wurden durch eigene Kinder, durch Bekannte und Verwandte sowie Medien und Internet über die Behandlungsmöglichkeiten in der Kieferorthopädie aufmerksam gemacht (Abbildung 3.5).

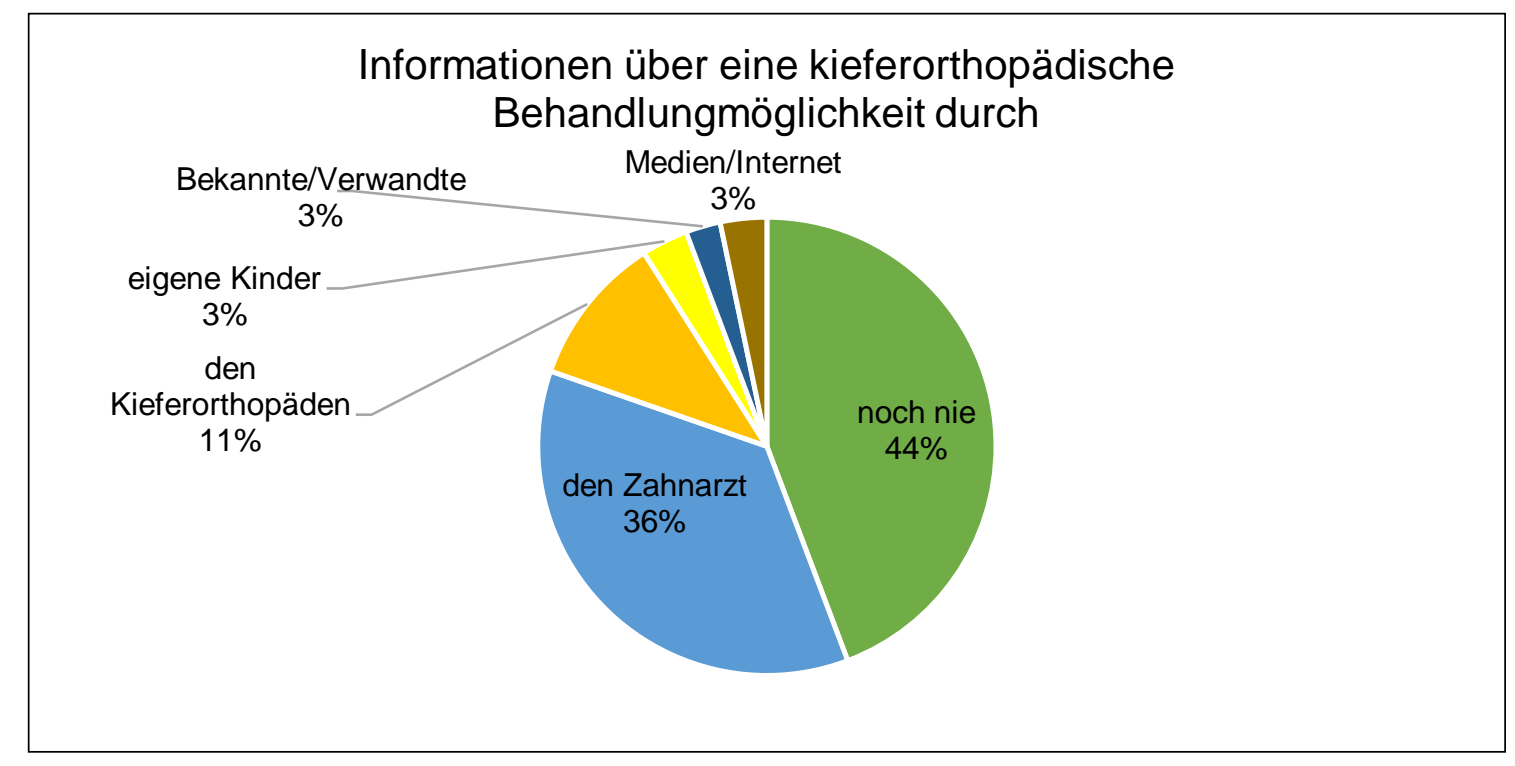

Abbildung 3.5: Kreisdiagramm zur Verteilung des Informationsgewinns über die kieferorthopädischen Behandlungsmöglichkeiten. 
3.6.4 Interesse an einer kieferorthopädischen Behandlung

In allen drei Gruppen war das Interesse hinsichtlich einer kieferorthopädischen Behandlung überwiegend verneint worden. Patienten mit einer moderaten oder schweren Parodontitis zeigten die Tendenz, eher an Behandlungen der Kieferorthopädie interessiert zu sein (Abbildung 3.6).

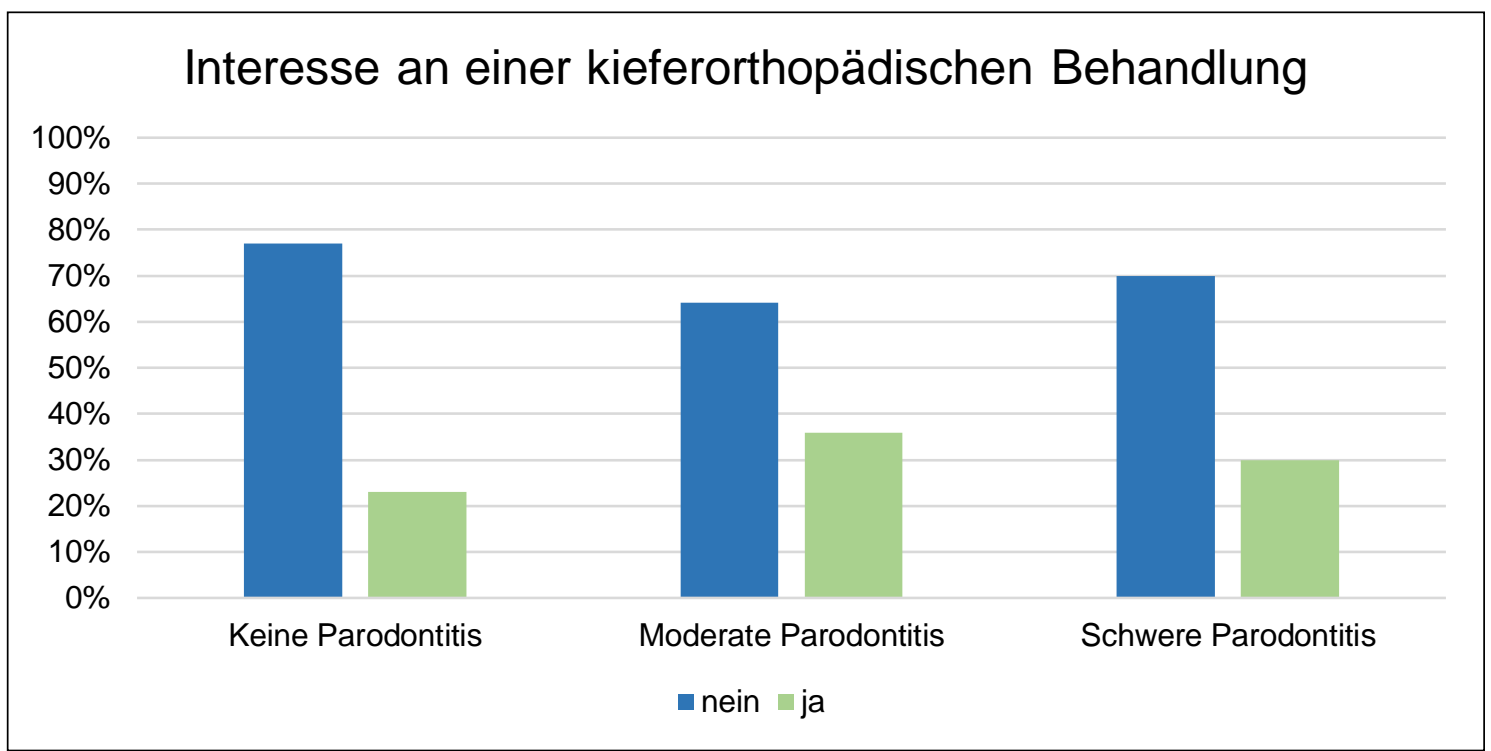

Abbildung 3.6: Das Balkendiagramm zeigt das Interesse an einer kieferorthopädischen Behandlung im Allgemeinen bezogen auf die Patientengruppen „keine Parodontitis“, „moderate Parodontitis“ und „schwere Parodontitis“. 


\subsubsection{Erwartungen an eine kieferorthopädische Behandlung}

Hinsichtlich der Erwartungen an eine kieferorthopädische Behandlung bezogen auf den langfristigen Erhalt der eigenen Dentition zeigte sich in allen drei Gruppen, dass hierauf großen Wert gelegt wird. $69 \%$ der Patienten ohne parodontale Erkrankung gaben an, hohe Erwartungen an eine kieferorthopädische Therapie zu haben, wenn es um den Erhalt der eigenen Zähne geht. In den Gruppen mit moderater und schwerer Parodontitis wurden von $77 \%$ beziehungsweise $82 \%$ der Befragten hohe Erwartungen an den Erhalt der eigenen Dentition angegeben (Abbildung 3.7).

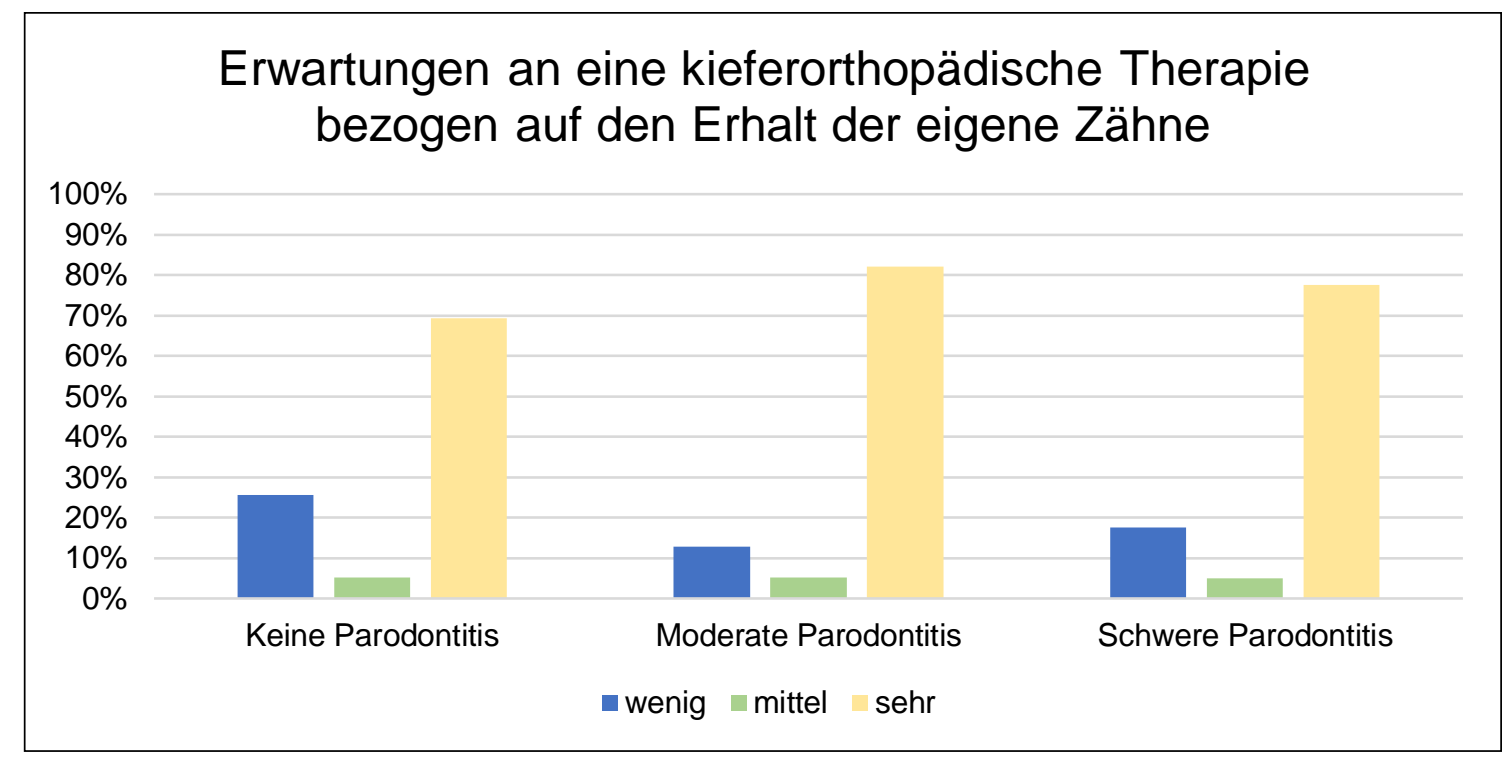

Abbildung 3.7: Das Balkendiagramm zeigt die Erwartungen an eine kieferorthopädische Behandlung bezogen auf den Erhalt der eigenen Zähne zwischen den Patientengruppen „keine Parodontitis“, „moderate Parodontitis“ und „schwere Parodontitis“ 
Die Erwartungen an eine kieferorthopädische Therapie bezogen auf die ästhetische Komponente waren bei Patienten mit moderater Parodontitis und schwerer Parodontitis wesentlich höher als bei Patienten ohne parodontale Erkrankung. Kein Patient ohne parodontale Destruktion erwartete eine ästhetische Verbesserung nach einer kieferorthopädischen Therapie. Hingegen gaben über $50 \%$ der moderat und $40 \%$ der schwer erkrankten Patienten an, große Erwartungen an die Ästhetik zu haben, würden sie sich einer kieferorthopädischen Therapie unterziehen (Abbildung 3.8).

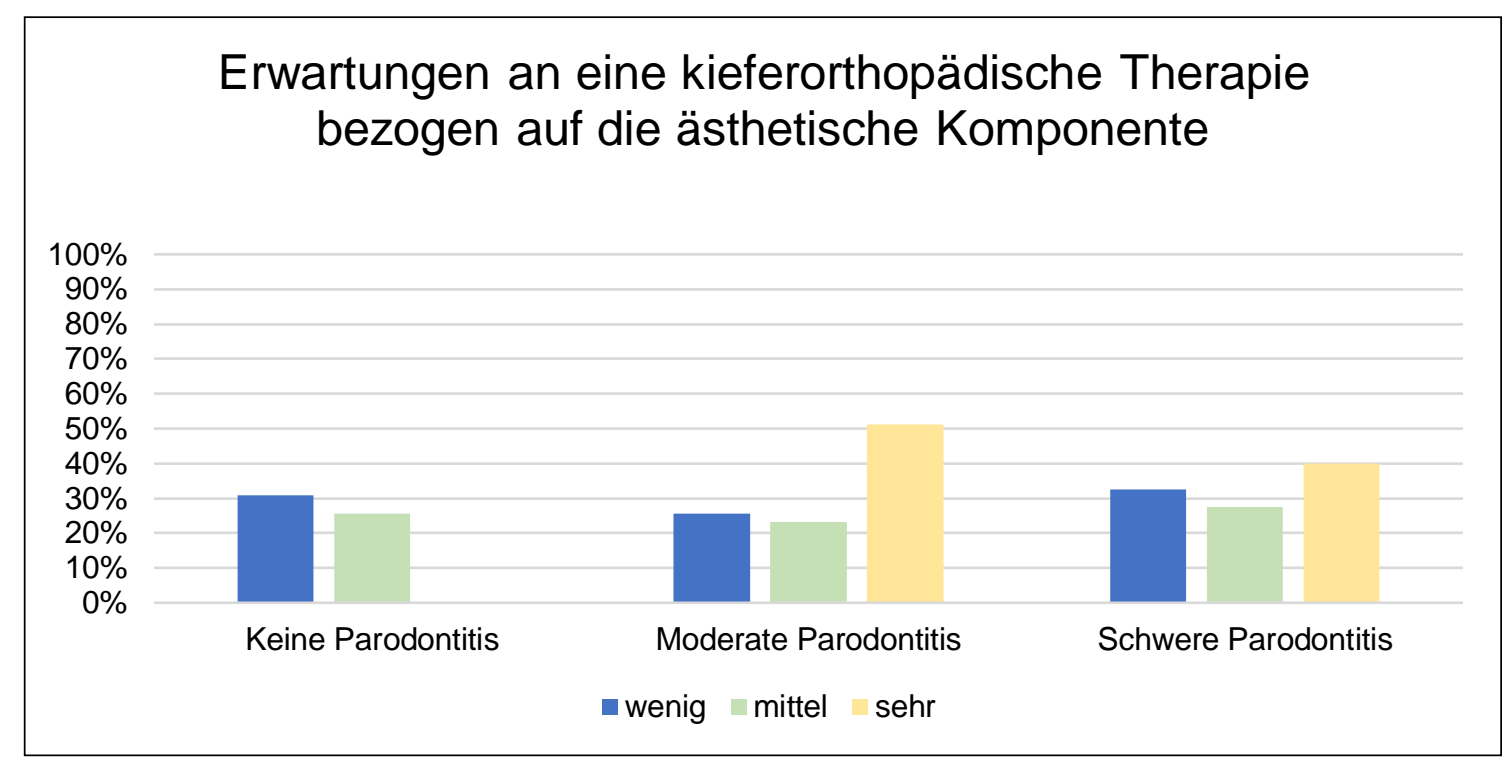

Abbildung 3.8: Das Balkendiagramm zeigt die Erwartungen an eine kieferorthopädische Behandlung bezogen auf die ästhetische Komponente der Patientengruppen „keine Parodontitis“, „moderate Parodontitis“ und „schwere Parodontitis“. 


\subsubsection{Ergebnisse oral health impact profile}

Die mundgesundheitsbezogene Lebensqualität wurde mit Hilfe des oral health impact profile (OHIP) erfasst. Aus den Antwortmöglichkeiten der 14 Fragen wurden Summenwerte gebildet, welche von 0-56 reichen konnten. Hohe Summenwerte lassen auf eine verminderte mundgesundheitsbezogene Lebensqualität schließen. Hinsichtlich der Summenwerte unterschieden sich die Patienten ohne Parodontitis signifikant von Patienten mit schwerer Parodontitis $(p=0,001)$. Patienten mit moderater Parodontitis unterschieden sich ebenfalls signifikant von Patienten mit schwerer Parodontitis $(p=0,008)$. Zwischen nicht erkrankten Patienten und moderat erkrankten waren keine Differenzen bezüglich der OHIP-Summe zu erkennen. Patienten mit schwerer Parodontitis hatten signifikant höhere OHIP-Summenwerte als in den anderen Gruppen. Dies lässt auf funktionelle und soziale Einschränkungen schließen, sowie auf eine generelle Unzufriedenheit mit der Mundgesundheit (Tabelle 3.11, Abbildung 3.9).

Tabelle 3.11: Vergleich der OHIP-Summe zwischen den Patientengruppen „keine Parodontitis“, „moderate Parodontitis“ und „schwere Parodontitis“. Die OHIP-Summen wurden als Mittelwert (MW) mit dazugehörigem 95 \%-Konfidenzintervall $(\mathrm{Cl})$ angegeben. Die Patientengruppen wurden mit Kruskal-WallisTest und Post-hoc Tests verglichen. Die p-Werte wurden mit der Bonferroni-Methode korrigiert.

\begin{tabular}{|c|c|c|c|c|c|c|c|}
\hline & \multicolumn{3}{|c|}{$\begin{array}{l}\text { Patienten- } \\
\text { gruppen }\end{array}$} & \multirow[t]{2}{*}{$\begin{array}{l}\text { Korr. } \\
\text { p-Wert }\end{array}$} & \multicolumn{3}{|c|}{$\begin{array}{l}\text { Post-hoc Test } \\
\text { Korr. p-Wert }\end{array}$} \\
\hline & $\begin{array}{c}\text { Keine } \\
\text { Parodontitis } \\
\text { MW } \\
\text { (Cl } 2,5 \% ; \\
\text { Cl } 97,5 \%) \\
\end{array}$ & $\begin{array}{c}\text { Moderate } \\
\text { Parodontitis } \\
\text { MW } \\
\text { (Cl 2,5\%; } \\
\text { Cl 97,5\%) } \\
\end{array}$ & $\begin{array}{c}\text { Schwere } \\
\text { Parodontitis } \\
\text { MW } \\
\text { (Cl 2,5\%; } \\
\mathrm{Cl} 97,5 \%) \\
\end{array}$ & & $\begin{array}{c}\text { Keine } \\
\text { vs. } \\
\text { Moderate } \\
\text { Parodontitis }\end{array}$ & $\begin{array}{c}\text { Keine } \\
\text { vs. } \\
\text { Schwere } \\
\text { Parodontitis }\end{array}$ & $\begin{array}{c}\text { Moderate } \\
\text { vs. } \\
\text { Schwere } \\
\text { Parodontitis }\end{array}$ \\
\hline $\begin{array}{l}\text { OHIP- } \\
\text { Summe }\end{array}$ & $\begin{array}{c}3,90 \\
(2,21 ; \\
5,58)\end{array}$ & $\begin{array}{c}5,28 \\
(3,21 ; \\
7,36)\end{array}$ & $\begin{array}{c}9,50 \\
(6,59 \\
12,41)\end{array}$ & 0,002 & 0,466 & 0,001 & 0,008 \\
\hline
\end{tabular}




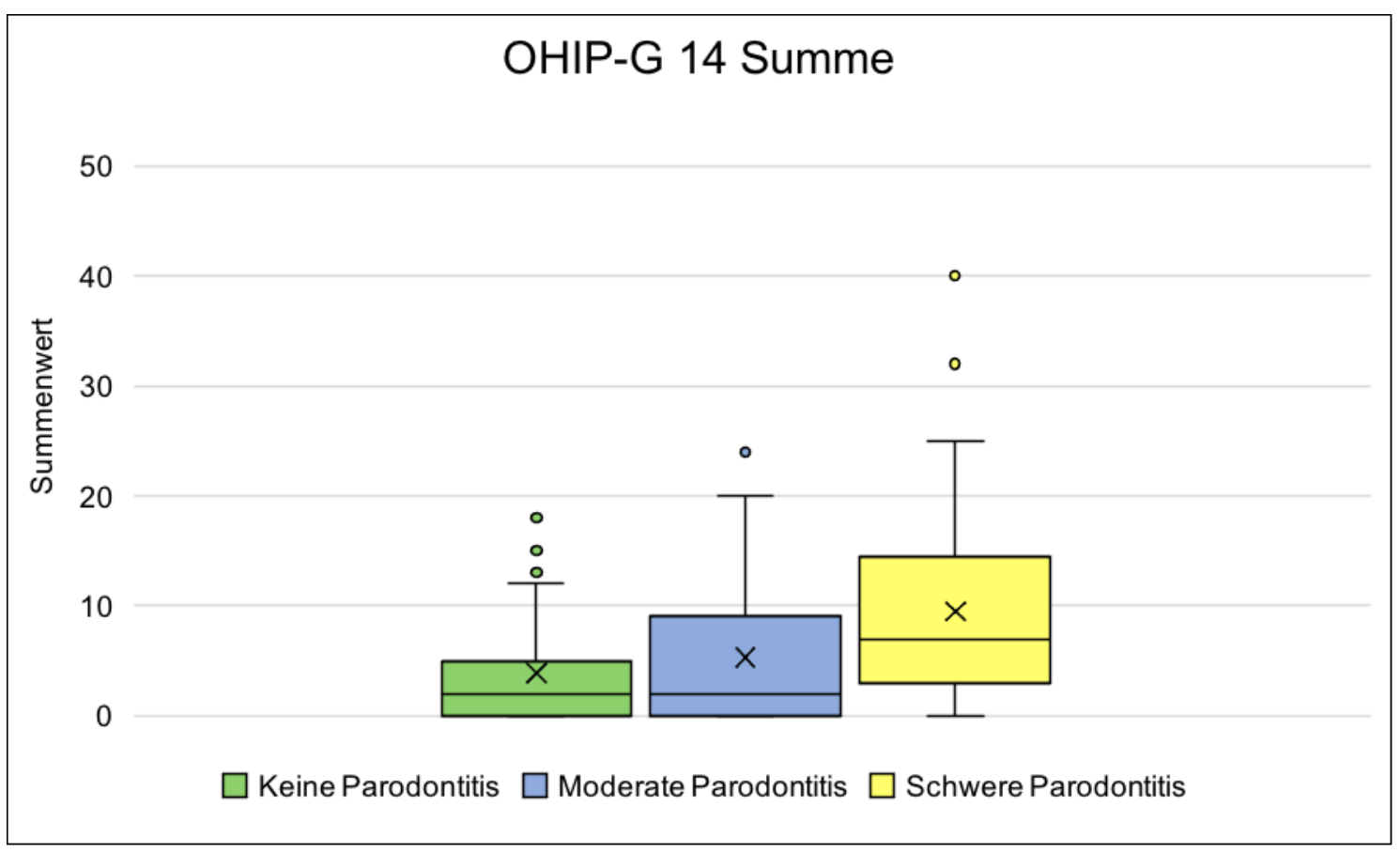

Abbildung 3.9: Die Box-Plots zeigten die OHIP-Summen zwischen den Patientengruppen „keine Parodontitis“, „moderate Parodontitis“ und „schwere Parodontitis“. 


\subsubsection{Ergebnisse general / geriatric oral health assessment index}

Der general / geriatric oral health assessment index (GOHAI) erfasst besonders bei einem älteren und parodontologisch kompromittierten Patientenkollektiv die Dimensionen der mundgesundheitsbezogenen Lebensqualität. Aus insgesamt zwölf Fragen wurden Summenwerte gebildet. Für die drei positiven Worthebungen wurden zuvor die Werte angepasst. Somit konnten Werte von 0 - 48 erreicht werden. Hohe Werte lassen auf eine Einschränkung der Lebensqualität schließen. Zwischen Patienten ohne parodontale Erkrankung und Patienten mit moderater Parodontitis sowie Patienten ohne parodontale Erkrankung und schwerer Erkrankung konnten signifikante Unterschiede aufgedeckt werden $(p=0,008 ; p<0,001)$. Außerdem unterschieden sich die Summenwerte der Patienten mit moderater und schwerer parodontaler Erkrankung signifikant $(p=0,012)$. Schwer erkrankte Patienten klagten in den letzten drei Monaten über Probleme bei der Nahrungsaufnahme, Einschränkungen im Umgang mit anderen Menschen, sowie Unsicherheit und Unzufriedenheit mit der Mundgesundheit (Tabelle 3.12, Abbildung 3.10).

Tabelle 3.12: Vergleich der GOHAI-Summe zwischen den Patientengruppen „keine Parodontitis“, „moderate Parodontitis“ und „schwere Parodontitis“. Die GOHAl-Summen wurden als Mittelwert (MW) mit dazugehörigem 95 \%-Konfidenzintervall $(\mathrm{Cl})$ angegeben. Die Patientengruppen wurden mit Kruskal-WallisTests und Post-hoc Tests verglichen. Die p-Werte wurden mit der Bonferroni-Methode korrigiert.

\begin{tabular}{|c|c|c|c|c|c|c|c|}
\hline & \multicolumn{3}{|c|}{$\begin{array}{l}\text { Patienten- } \\
\text { gruppen }\end{array}$} & \multirow[t]{2}{*}{$\begin{array}{l}\text { Korr. } \\
\text { p-Wert }\end{array}$} & \multicolumn{3}{|c|}{$\begin{array}{l}\text { Post-hoc Test } \\
\text { Korr. p-Wert }\end{array}$} \\
\hline & $\begin{array}{c}\text { Keine } \\
\text { Parodontitis } \\
\text { MW } \\
\text { (Cl 2,5\%; } \\
\mathrm{Cl} 97,5 \%) \\
\end{array}$ & $\begin{array}{c}\text { Moderate } \\
\text { Parodontitis } \\
\text { MW } \\
\text { (CI 2,5\%; } \\
\text { Cl 97,5\%) } \\
\end{array}$ & $\begin{array}{c}\text { Schwere } \\
\text { Parodontitis } \\
\text { MW } \\
\text { (CI 2,5\%; } \\
\text { Cl 97,5\%) } \\
\end{array}$ & & $\begin{array}{c}\text { Keine } \\
\text { vs. } \\
\text { Moderate } \\
\text { Parodontitis }\end{array}$ & $\begin{array}{c}\text { Keine } \\
\text { vs. } \\
\text { Schwere } \\
\text { Parodontitis }\end{array}$ & $\begin{array}{c}\text { Moderate } \\
\text { vs. } \\
\text { Schwere } \\
\text { Parodontitis }\end{array}$ \\
\hline $\begin{array}{l}\text { GOHAI- } \\
\text { Summe }\end{array}$ & $\begin{array}{c}6,21 \\
(4,45 \\
7,96)\end{array}$ & $\begin{array}{c}9,21 \\
(7,37 ; \\
11,05)\end{array}$ & $\begin{array}{c}13,13 \\
(10,64 ; \\
15,61)\end{array}$ & $<0,001$ & 0,008 & $<0,001$ & 0,012 \\
\hline
\end{tabular}




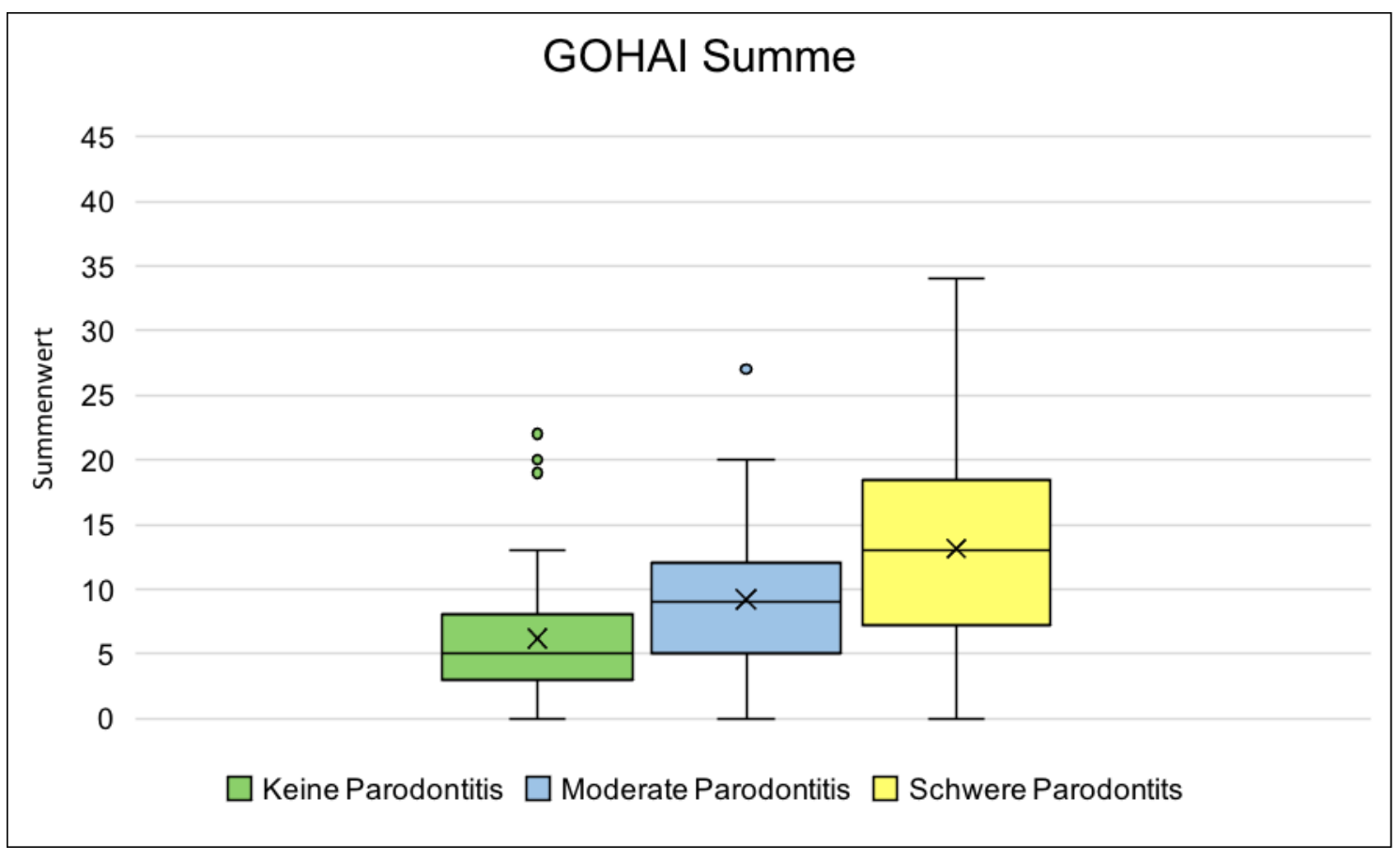

Abbildung 3.10: Die Box-Plots zeigen die GOHAI-Summen der Patientengruppen „keine Parodontitis“, „moderate Parodontitis“ und „schwere Parodontitis“. 


\section{Diskussion}

\subsection{Diskussion der Methode}

Für die vorliegende Studie wurden weibliche und männliche Patienten im Alter von 40 bis 84 Jahren untersucht. Diese Altersgruppe ist typischerweise von parodontalen Erkrankungen im Erwachsenenalter betroffen (Albandar 2005; Van Dyke und Sheilesh 2005; Schiffner 2016). Die Erhebung der Indizes, Aufnahme der dreidimensionalen Bilder und Bearbeitung der Fragebögen fand unter standardisierten Bedingungen statt. Es mussten acht Patienten aufgrund ihres Zahnstatus oder qualitativ schlechter Scaninformationen ausgeschlossen werden. Durch wöchentliche Kalibrierung des Oberflächenscanners und Modellscanners, sowie Erfahrung des Behandlers wurden Messabweichungen minimiert (Gwilliam et al. 2006).

In den kommenden Jahren und Jahrzehnten ist davon auszugehen, dass Patienten mit parodontalen Erkrankungen und pathologischen Zahnwanderungen vermehrt im kieferorthopädischen und zahnärztlichen Behandlungsalltag behandelt werden, da in alternden Populationen ein längerer Erhalt der eigenen Dentition beobachtet wird (Edman et al. 2015).

In dieser Studie wurde erstmals im Rahmen einer kieferorthopädischen Diagnostik eine Patientengruppe von ausschließlich älteren Erwachsenen $\geq 40$ Jahre untersucht und zusätzlich in parodontal gesund, moderat und schwer erkrankt klassifiziert. Deren parodontaler Status, die Malokklusion und die mundgesundheitsbezogene Lebensqualität sowie das kieferorthopädische Behandlungsinteresse und der Bedarf wurden quantifiziert und zur parodontalen Destruktion in Bezug gesetzt. Außerdem wurde ein dreidimensionales Patientenmodell zur Darstellung der Ausgangssituation und späteren Verlaufskontrolle erstellt.

\subsubsection{Einteilung der Gruppen}

Die Patientenakquise erfolgte anhand der definierten Ein- und Ausschlusskriterien. Patienten mit Fehlbildungen des Gesichts und bestehenden schwerwiegenden Malokklusionen wurden ausgeschlossen, um eine Verfälschung der Ergebnisse weitgehend zu vermeiden. Die Besonderheit des 
kieferorthopädischen Patientengutes liegt an der Definition des Mindestalters von 40 Jahren. Somit wurde zum einen dem aktuell steigenden kieferorthopädischen Patientenalter entsprochen, und zum anderen die steigende Prävalenz von Parodontitis mit zunehmendem Alter berücksichtigt (Beck et al. 1990; Van Dyke und Sheilesh 2005). Das ausgewählte Patientenkollektiv hatte ein mittleres Alter von 58 Jahren. Diese Verteilung spiegelt den demografischen Wandel und die Verlagerung der parodontalen Erkrankung in ein höheres Lebensalter sowie den langen Erhalt der eigenen Zähne wider (Schiffner 2016; Chapple et al. 2018). Der Anteil der weiblichen Patienten in der Gruppe ohne parodontale Erkrankung war höher, als der der männlichen Patienten. Dies entspricht den Beobachtungen von Grossi et al. (1995), die einen Zusammenhang zwischen dem männlichen Geschlecht und der parodontalen Destruktion in einem niedrigeren Lebensalter zeigen konnten. Der Frauenanteil einer Motivationsstudie zur kieferorthopädischen Behandlung lag mit $66 \%$ ebenfalls etwas über dem der männlichen Teilnehmer (Bauer und Diedrich 1990). Da es sich bei den drei Gruppen dieser Studie um zufällig ausgewählte Patienten handelte, welche sich in einem Recallprogramm befanden, können die Ergebnisse weitgehend auf die Allgemeinbevölkerung übertragen werden. Möglicherweise sind diese Patienten durch die Teilnahme an einem Recallprogramm jedoch als motivierter zu erachten.

Für eine belastbare Einteilung der Patienten hinsichtlich des Ausprägungsgrades der Parodontitis erfolgte die Gruppierung nach Erkenntnissen der CDC-Working Group in Zusammenarbeit mit der American Academy of Periodontology (Page und Eke 2007). Es wurden zahnmedizinisch etablierte Indizes erhoben, die den Erkrankungsgrad kategorisieren und gut reproduzierbar sind (Armitage 1996; Greenstein 1997). Andere Studien, welche sich mit der Einteilung des Schweregrades der Parodontitis beschäftigten, verwendeten ebenfalls die etablierte Einteilung der American Academy of Periodontology (Holtfreter et al. 2009; Konig et al. 2010; Holtfreter et al. 2015; Hirschfeld et al. 2019)

\subsubsection{Klinische Datenerhebung und Modellanalyse}

Für die zugrundeliegende Studie wurde für jeden Patienten ein ausführlicher klinischer Befund aufgenommen. Hierbei wurden die Sondierungstiefen und Rezessionen gemessen und der clinical attachment loss daraus ermittelt. Die 
Erhebung von Sondierungstiefen ist in anderen klinischen und epidemiologischen Studien die am häufigsten verwendete Methode und kann somit als etabliert angesehen werden (Listgarten 1980; Hefti 1997). Erwachsene Patienten sind häufig nicht mehr vollbezahnt, deshalb ist es schwierig umfassendere Einschlusskriterien zu definieren. In der vorliegenden Studie wurden so 72 Messpunkte von Eckzahn bis Eckzahn in Ober- und Unterkiefer für jeden Patienten ermittelt und als ausreichend definiert. Wohingegen die Falldefinition der CDC Working Group nur die dritten Molaren ausgeschlossen hat. Für zukünftige Studien könnte man die klinisch-parodontalen Parameter an allen Zähnen erheben. Es muss aber auf die Vergleichbarkeit der Daten, hinsichtlich Zahnanzahl und Position geachtet werden. Im kieferorthopädischen Praxisalltag stellen sich erwachsene Patienten mit parodontaler Erkrankung häufig auf Grund von pathologischen Zahnwanderungen im Frontzahnbereich vor. Diese ästhetischen Veränderungen sowie funktionelle Einschränkungen wie beispielsweise ein inkompetenter Lippenschluss veranlassen Patienten sich kieferorthopädisch beraten und behandeln zu lassen. Deswegen sollte in dieser Studie vorallem der Frontzahnbereich untersucht werden. Die Auswertung beziehungsweise Gruppierung der Befunde erfolgte nach den Kriterien der CDC Working Group (Page und Eke 2007).

Darüber hinaus wurde der Lockerungsgrad zur Darstellung parodontalen Destruktion erfasst. Frühere Studien teilten ebenfalls verschiedene Patientengruppen anhand der parodontalen Befunde ein (Havemose-Poulsen et al. 2006; de Pablo et al. 2008; Pischon et al. 2008; Müller Campanile et al. 2019). Anders als in der vorliegenden Arbeit wurden dabei lediglich Sondierungstiefen erhoben und nicht der clinical attachment loss. Dadurch kann es zu einer Unterschätzung der Parodontitis kommen. Deshalb addierte man in dieser Studie Sondierungstiefen und Rezessionen zum clinical attachment loss.

Der clinical attachment loss wurde auch in anderen Studien berücksichtigt, weil dieser die tatsächliche parodontale Situation exakter darstellt (Holtfreter et al. 2015; Poulsen et al. 2019). Die Reproduzierbarkeit von Messungen der Sondierungstiefen mit der Parodontalsonde PCPUNC15 zeigt in der Literatur klinisch vertretbare Genauigkeiten. Diese wurde auch in der vorliegenden Studie verwendet, da sie mit ihrer Millimeterskalierung als Goldstandard angesehen wird (Glavind und Loe 1967; Wang et al. 1995; Holtfreter et al. 2012). Andere Autoren 
verwendeten Röntgenaufnahmen, um den Knochenverlauf zu quantifizieren und den Schweregrad der Parodontitis zu klassifizieren (Helenius et al. 2005; Albandar 2014).

Die Kombination aus Röntgenaufnahmen und clinical attachment loss bringt den größten Informationsgewinn. Da in dieser Studie aus ethischen Gründen und zum Schutz der Patienten auf zusätzliche Röntgenaufnahmen verzichtet wurde, entfiel diese Methodik.

Mit der Erhebung der Modellparameter (overjet, overbite, Little-Index OK / UK, IOTN-DHC) in Bezug zu den Patientengruppen sollten Zusammenhänge zwischen dem Malokklusionsgrad und der Parodontitis dargestellt werden. Die definierten Parameter stellen in der alltäglichen kieferorthopädischen Praxis etablierte Analysewerkzeuge dar, um Zahnfehlstellungen zu quantifizieren. So finden sich national ähnliche Analysewerkzeuge in der sogenannten KIG-Tabelle (Richtlinien des Bundesausschusses der Zahnärzte und Krankenkassen für die kieferorthopädische Behandlung 2003). Ähnliche Parameter wurden zur Quantifizierung eines kieferorthopädischen Behandlungsbedarfs beziehungsweise zur Befundung der Malokklusion in anderen Studien angewandt (Hensel et al. 2003; Ngom et al. 2006; Bernhardt et al. 2019; Hirschfeld et al. 2019). Der in dieser Arbeit verwendete IOTN stellt ein international weit verbreitetes Instrument zur Festlegung des Malokklusionsgrades dar. Durch Verwendung dieses Index lassen sich die Ergebnisse leicht mit internationalen Studien vergleichen.

\subsubsection{Digitale Datengenerierung und Erstellung des virtuellen Patienten}

Die Oberflächenscans des Gesichtsweichteils wurden mit dem Vectra ${ }^{\circledR}$ N5 Head Scanner (Version 7.4.1, Canfield Scientific, Parsippany, NJ, USA) generiert. Dieser wurde in etablierten Studien zuvor bereits verwendet (Sawyer et al. 2009; Rosati et al. 2012; Masoud et al. 2017). Dabei wurde das Prinzip der Stereofotogrammmetrie genutzt, welches eine schnelle Datenverarbeitung und kurze Aufnahmezeit ermöglicht und somit praxistauglich ist. Ein Scan wurde mit geschlossenem Mund und der Dentition in maximaler Interkuspidation erstellt, sowie ein weiterer Scan, bei dem der Patient Wangenabhalter zur besseren Darstellung der Dentition positionierte. Um Lichtreflektionen zu minimieren, wurde hierbei ein Mattierungspuder aufgetragen. Vergleichbare Studien, welche 
das Gesichtsweichteil analysierten, verwendeten ebenfalls Wangenabhalter und Mattierungsspray (Rangel et al. 2008; Rangel et al. 2016). Schon frühere Studien generierten den virtuellen Patienten durch die Digitalisierung der analogen Gipsmodelle (Rangel et al. 2008; Rosati et al. 2012; Masoud et al. 2017; Rangel et al. 2018). Bei der Digitalisierung der Gipsmodelle ist kein Informationsverlust beziehungsweise Dimensionsverlust zu erwarten (De Luca Canto et al. 2015; Rossini et al. 2016). Frühere Studien nutzten ebenfalls ein dreidimensionales Patientenmodell mit individuell definierten landmarks, um morphologische Veränderungen und Zustände sowie ethnische Merkmale zu dokumentieren. Auch zur Veränderung von Weichteilen durch Zahnbewegungen wurden Untersuchungen durchgeführt (Sawyer et al. 2009; Kim et al. 2014; Galantucci et al. 2016; Ceinos et al. 2017; Celebi et al. 2017; Jang et al. 2017; Masoud et al. 2017). Die Reproduzierbarkeit des Setzens von definierten anatomischen Punkten, sogenannte landmarks, ist für nachfolgende Auswertungen wichtig. Sie liegt in einigen Studien in einem klinisch akzeptablen Bereich von unter $1 \mathrm{~mm}$. Hierbei ist die Verwendung eines kartesischen Koordinatensystems, bestehend aus $x-, y-$, und z-Achse, sowie eine exakte Definition und Beschreibung der landmarks, von Bedeutung (Gwilliam et al. 2006; Toma et al. 2009). Es ist für die Vergleichbarkeit und Reproduzierbarkeit von Messungen zu verschiedenen Zeitpunkten besser, wenn nur ein Behandler die Analyse durchführt. Die Erfahrung des Behandlers mit der Analysesoftware ist von besonderer Wichtigkeit. Ein geschulter Behandler kann landmarks besser reproduzieren (Gwilliam et al. 2006). Durch Verwendung dieser etablierten Methoden ist von einer korrekten Generierung der virtuellen Daten auszugehen. 


\subsection{Diskussion der Ergebnisse}

\subsubsection{Klinisch-parodontale Parameter}

Die klinisch-parodontalen Parameter dienten der Gruppeneinteilung. Der Lockerungsgrad nimmt mit der Schwere der Parodontitis stetig zu. Es sind signifikante Unterschiede zwischen nicht erkrankten Patienten und moderat erkrankten zu schwer erkrankten Patienten zu erkennen. Dies stimmt mit Ergebnissen aus anderen Studien überein (Towfighi et al. 1997; Brunsvold 2005; Pihlstrom et al. 2005). Insgesamt war die parodontale Destruktion eher moderat einzustufen. Der Mittelwert bei einer schweren Parodontitis für den CAL lag für den Oberkiefer bei 3,32 mm. Für zukünttige Studien könnte es sinnvoll sein Subgruppen mit schwereren Ausprägungsgraden der Parodontitis zu bilden. Die insgesamt moderaten Befunde der klinisch-parodontalen Parameter könnten ausschlaggebend dafür sein, dass die Weichteilbefunde und orthodontischen Parameter sich nicht zwischen allen Gruppen unterschieden.

\subsubsection{Orthodontische Parameter}

Die orthodontischen Parameter quantifizieren den Grad der Malokklusion und den Behandlungsbedarf anhand kieferorthopädischer Gesichtspunkte. Der Malokklusionsgrad bei Patienten wurde schon in anderen Studien bestimmt und mit den parodontalen Befunden in Zusammenhang gebracht (Ngom et al. 2006; Bernhardt et al. 2019). Allerdings konnten mit dieser Arbeit erstmals Daten über ein älteres Patientenkollektiv dargestellt werden. Die wenigen bisherigen Studien im Bereich der Erwachsenenkieferorthopädie stützen sich auf deutlich jüngere Patientengruppen (Bernhardt et al. 2019).

Die Schwere der parodontalen Destruktion zeigte sich auch in den orthodontischen Befunden. Patienten mit einer schweren Parodontitis zeigten signifikant häufiger pathologische Vergrößerungen der Frontzahnstufe (overjet). Die Kontaktpunktabweichungen in der Oberkiefer- und Unterkieferfront unterschieden sich signifikant zwischen parodontal gesunden Patienten und solchen mit schwerer Parodontitis. Dies lässt Rückschlüsse auf Zahnmigrationen im Frontzahnbereich zu, welche im Zusammenhang mit einer schweren Parodontitis auftreten. Es muss jedoch berücksichtigt werden, dass 
Kontaktpunktabweichungen innerhalb der Oberkiefer- und Unterkieferfront auch durch tertiäre Engstände entstehen können (van der Linden 1975).

Der IOTN-DHC stieg stetig mit Zunahme des parodontalen Erkrankungsgrades an. Patienten mit einer schweren Parodontitis leiden demnach signifikant häufiger an einer gestörten Okklusion sowie funktionellen und ästhetischen Einschränkungen.

Pathologische Zahnwanderungen treten dann auf, wenn ein Missverhältnis zwischen den orofazialen Komponenten entsteht. Die parodontale Destruktion als Prädilektionsfaktor für die Entstehung pathologischer Zahnmigrationen ist an erster Stelle zu nennen, gefolgt von einer ungesicherten Okklusion und pathologischen Weichteilkräften (Proffit 1978; Chasens 1979; Towfighi et al. 1997; Brunsvold 2005). Der IOTN-DHC hat allerdings seine Grenzen bei der Untersuchung der zugrunde liegenden Kohorte, da er ursprünglich für ein jüngeres Patientenkollektiv ausgelegt wurde. Für die Beurteilung eines älteren Patientenklientels mit pathologischen parodontalen Befunden sollte der Index in Zukunft angeglichen werden. Allerdings existieren bislang keine Indizes für das hier untersuchte Patientenkollektiv.

Eine kieferorthopädische Behandlung kann durch korrektes Ausrichten geneigter oder pathologisch gewanderter Zähne die parodontale Therapie erleichtern und einem Rezidiv vorbeugen. Dadurch kann eine gesicherte, funktionell stabile Okklusion erzielt werden. Diese ist von großer Bedeutung für den langfristigen Erfolg einer parodontalen Therapie (Stahl 1975; Caffesse 1980; Ramfjord und Ash 1981; Gher 1998; van Gastel et al. 2007). Eine Studie von van Gastel et al. (2007) konnte zeigen, dass es nur in sehr seltenen Fällen zu einer Verschlechterung der parodontalen Situation während einer kieferorthopädischen Therapie kommt. Nicht außer Acht zu lassen sind die Auswirkungen der Malokklusion auf psychische Komponenten. Patienten mit deutlich pathologischen orthodontischen Befunden und einem hohen IOTN-DHC waren allgemein unzufriedener mit ihrer Mundgesundheit. Vorangegangene Studien konnten ebenfalls eine Beeinträchtigung der Zufriedenheit mit der dentalen Ästhetik feststellen. Fehlende Zähne und Unregelmäßigkeiten im Frontzahnbogen wurden dabei als Einflussfaktoren identifiziert (Horup et al. 1987; Borges et al. 2010; Tessarollo et al. 2012) 


\subsubsection{Dreidimensionale Analyse / Virtuelles Patientenmodell}

Die dreidimensionale Analyse basierte auf einem virtuell erstellten Patientenmodell. Andere Studien hatten ebenfalls zum Ziel, einen virtuellen Patienten, bestehend aus Gesichtsweichteil und digitalem Modell, zu erstellen (Rangel et al. 2008; Rosati et al. 2010; Rosati et al. 2012). Damit bessere Scanergebnisse erreicht werden konnten, wurde ein Mattierungsspray auf die glänzende Zahnoberfläche aufgetragen (Rangel et al. 2016). Dennoch wurden bei deutlichen verschachtelt stehenden Zähnen schlechtere Scanergebnisse erzielt, als bei harmonisch ausgeformten Zahnbögen. Die Erfassung glänzender und spiegelnder Oberflächen muss verbessert werden, damit in Zukunft zuverlässigere Scanergebnisse erzielt werden können.

In der Studie von Rangel et al. (2008) konnte eine Überlagerungsgenauigkeit mit 0,35 mm maximalem Abstand zwischen den dreidimensionalen Objekten erreicht werden. In der vorliegenden Studie wurde die Überlagerungspräzision mit einem Intra-Rater-Reliabilitätskoeffizienten bestimmt. Es konnten sehr gute, signifikante Vorhersagewerte für die zweite Messung erreicht werden. Außerdem wurden exaktere Annäherungen der Objekte als in vorangegangenen Studien erreicht. Diese geringen Abstände zwischen den Objekten sind klinisch nicht relevant (Rangel et al. 2008).

Die Ergebnisse der Zahnfehlstellungen wurden in Bezug zum parodontalen Erkrankungsgrad gesetzt. Es konnten keine signifikanten Zusammenhänge zwischen Weichteilveränderungen und dem Schweregrad der Parodontitis aufgedeckt werden. Belastbare Parameter müssen etabliert werden, damit es möglich wird, Weichteilveränderungen besser in die kieferorthopädische Planung einzubeziehen.

Auf Grund des untersuchten breiten Patientenklientels konnten keine Zusammenhänge zwischen der parodontalen Destruktion und Weichteilanalyse hergestellt werden. Hierfür wären wahrscheinlich weitere Subklassifikationen der Patientengruppen notwendig. So könnte die Unterteilung in die skelettale Konfiguration in der sagittalen, transversalen und vertikalen Dimension gegebenenfalls weitere Hinweise liefern. Die Ergebnisse von Joshi et al. (2015) zeigten einen signifikanten Unterschied in der sagittalen Lippenposition bei verschiedenen skelettalen Anomalien. Zudem sind für jede der skelettalen 
Konfigurationen unterschiedliche Referenzlinien zur Analyse der Lippenposition heranzuziehen. Außerdem konnten Duran et al. (2019) zeigen, dass bei Patienten mit einer skelettalen Klasse III häufiger Asymmetrien auftreten die wiederum die Lippenmorphologie beeinflussen können.

In der vorliegenden Studie wurde auch nicht zwischen dem Geschlecht, der Größe des Patienten, dem Body-Mass-Index und dem Alter differenziert. Die genannten Parameter nehmen aber erheblichen Einfluss auf das Gesichtsweichteil und sollten in zukünftigen Studien beachtet werden.

Allerdings zeigt das in dieser Arbeit etablierte Verfahren bezüglich der Erstellung eines virtuellen Patienten ein sehr gut nutzbares Modell für Verlaufskontrollen bei kieferorthopädischer Therapie und kann in Zukunft genutzt werden. Vor allem bei Patienten mit pathologisch gewanderten Frontzähnen, deutlich vergrößertem overjet und aufgefächerten Fronten verändert sich im Laufe der Therapie das Gesichtsweichteil und in Folge dessen das Gesichtsprofil. Der Vergleich prätherapeutischer und posttherapeutischer Weichteilbefunde wurde schon in anderen Studien durchgeführt. Dabei wurden Veränderungen des Weichteilprofils nach einer Vielzahl von Frontzahnbewegungen in verschiedenen Richtungen bei erwachsenen Patienten untersucht. Hierbei diente ein palatinales Implantat als stabiler Referenzpunkt. Es konnten signifikante Korrelationen zwischen horizontalen Veränderungen der Schneidezähne und der horizontalen Position der Oberlippe und Unterlippe festgestellt werden (Kuhn et al. 2016). In der vorliegenden Studie fehlte ein solcher fixer Referenzpunkt. Außerdem wurden Patienten untereinander verglichen und nicht jeder Patient isoliert, vor und nach der Therapie. Dies könnte in zukünftigen Studien vertieft werden, damit sowohl gezeigt werden kann, dass eine optimierte Zahnstellung durch kieferorthopädische Behandlung erreicht werden kann aber auch eine funktionell und ästhetisch ansprechende Weichteilmorphologie zu erreichen ist. Eine Studie von Kim et al. (2014) bekräftigt die Wichtigkeit der Analyse von Weichteilveränderungen im Verlauf der kieferorthopädischen Behandlung bei dem jeweiligen Patienten. Durch Kompositapplikation auf die Labialfläche der Zähne wurden Veränderungen der Lippenmorphologie dokumentiert. In der vorliegenden Studie konnten Auffächerungen, Extrusion und Labialkippungen der Frontzähne bei starker parodontaler Destruktion festgestellt werden. Patienten mit diesen Befunden hatten Probleme beim Lippenschluss und das 
Profil dieser Patienten hatte sich verschlechtert. Interessant wäre hier zu überprüfen, wie sich das Lippenprofil nach Intrusion und Retrusion des Frontzahnsegments posttherapeutisch verändert hat.

Zur Kontrolle des Behandlungserfolgs und besseren Visualisierung für den Patienten wird die in der Mund-Kiefer- und Gesichtschirurgie schon lange von der dreidimensionalen Oberflächendarstellung Gebrauch gemacht (Schwenzer et al. 1998; Joss et al. 2010). Für nachfolgende Studien sollten bei Patienten prä- und posttherapeutische Daten des Gesichtsweichteils analysiert werden. Nach Kopplung der Daten mit der kieferorthopädischen Therapie sollten Aussagen, unter anderem bezüglich der Vorhersagbarkeit der Gesichtsweichteilveränderungen infolge kieferorthopädischer Behandlungen, möglich sein.

\subsubsection{Fragebögen}

Eine Steigerung des Bewusstseins für Zähne, Mund und Mundgesundheit konnte in Folge der parodontalen Therapie festgestellt werden. Die Veränderungen wurden von ca. $82 \%$ der Befragten als deutlich bis sehr deutlich beschrieben. Die Mundhygiene kann mit erfolgreicher Mundhygieneerziehung durch geschultes Personal und bedarfsorientierter Motivation verbessert werden (Zimmer et al. 2001; Nakre und Harikiran 2013). Patienten mit einer parodontalen Erkrankung können durch die Wahrnehmung eines Prophylaxeprogramms für inre Erkrankung sensibilisiert werden. Es liegt jedoch oft ein unzureichendes Wissen über Ätiologie, Symptome und damit verbundenen Risikofaktoren oder parodontalen Risikofaktoren bei Patienten vor (Varela-Centelles et al. 2019). In einer Studie von Deinzer et al. (2009) konnte gezeigt werden, dass das Wissen über Parodontitis in der deutschen Gesellschaft mit großen Defiziten einhergeht. Besonders bildungsschwache Menschen haben wenige Informationen über die Erkrankung. Umso wichtiger ist es in Zukunft, seitens der Zahnärzte und des zahnärztlichen Personals für eine bessere Aufklärung zu sorgen.

Es konnte beobachtet werden, dass ein Aufklärungsmangel auch hinsichtlich kieferorthopädischer Therapiemöglichkeiten besteht. Annähernd die Hälfte der Patienten wurde noch nie über kieferorthopädische Behandlungsmaßnahmen informiert. Im Jahr 1990 wurden in einer Motivationsstudie für erwachsene Probanden im Hinblick auf kieferorthopädische Behandlungen ähnliche 
Ergebnisse verzeichnet. Hier fehlte einem Drittel der Befragten die Information über Möglichkeiten der Kieferorthopädie. Als Hauptinformationsquelle wurde der Zahnarzt angegeben (Bauer und Diedrich 1990). Während der Befragung konnte festgestellt werden, dass der Begriff „Kieferorthopädie“ bei einem Großteil der Bevölkerung fälschlicherweise mit einer anderen Disziplin der Zahnmedizin assoziiert wird. Es wurden kieferchirurgische, aber auch prothetische Bezüge hergestellt.

Dies zeigt, wie wichtig es ist, den Patienten für Möglichkeiten und Grenzen der zahnmedizinischen, respektive kieferorthopädischen Therapie zu informieren und sensibilisieren. Aufgrund des hoch komplexen therapeutischen Aufwands bei der kieferorthopädischen Therapie parodontal kompromittierter Patienten werden viele dieser Fälle mittels Extraktion behandelt (Brunsvold 2005). Ein erhöhtes Behandlungsaufkommen für Patienten mit Parodontitis ist in der Zukunft durch längeren Erhalt der eigenen Dentition und den demographischen Wandel zu erwarten (Schutzhold et al. 2015; Schiffner 2016). Die Herausforderungen für die Kieferorthopädie in der Erwachsenenbehandlung werden weiter steigen (Wehrbein et al. 2011). Im Jahr 2004 wurden bereits $20 \%$ aller kieferorthopädischen Therapien bei einem erwachsenen Patientenklientel durchgeführt (Musich 2004).

Die kombiniert kieferorthopädisch-parodontologische Therapie kann nicht nur Zahnstellungen, Zahn- und Mundgesundheit verbessern, sondern auch ein besseres Selbstvertrauen und psychologisches Wohlbefinden fördern (Hunt et al. 2001; Johal et al. 2015; de Couto Nascimento et al. 2016). Die Gründe für Patienten, sich kieferorthopädisch behandeln zu lassen, sind Zahnfehlstellungen und eine Disharmonie zwischen Dentition und Gesichtsweichteil (Espeland und Stenvik 1991; McKiernan et al. 1992). Patienten mit schwerer parodontaler Destruktion leiden häufig unter Rezessionsbildungen, Lockerungen der Zähne, pathologischen Zahnwanderungen und lückig aufgefächerten Fronten, sowie einem ästhetisch beeinträchtigten Erscheinungsbild (Towfighi et al. 1997; Brunsvold 2005). Das ,jugendliche Lächeln“ als Sinnbild für Attraktivität spielt eine wichtige Rolle für das Selbstbewusstsein im psychosozialen Umfeld der Patienten (Diedrich 1992). In dieser Studie waren von den nicht an einer Parodontitis erkrankten Patienten nur etwa $20 \%$ an einer kieferorthopädischen Behandlung interessiert. Aus der Gruppe der moderat und schwer erkrankten 
Patienten war annähernd ein Drittel einer kieferorthopädischen Behandlung gegenüber aufgeschlossen. Eine Tendenz für den erhöhten Wunsch an einer Zahnstellungskorrektur mit Zunahme des Erkrankungsgrades ist zu verzeichnen. Dies kann durch das gesteigerte Bewusstsein für Zähne, Zahn- und Mundgesundheit bei Patienten mit einer Parodontitistherapie begründet sein. Dennoch sind verhältnismäßig wenig Patienten an einer Therapie der Kieferorthopädie interessiert, obwohl sie signifikant höhere Behandlungsbedürftigkeiten und Malokklusionen aufweisen. Hierfür könnte die mangelnde Aufklärung über Möglichkeiten der kieferorthopädischen Therapie eine Erklärung sein (Deinzer et al. 2009). Ein weiterer Erklärungsansatz besteht darin, dass viele ältere Patienten nicht bereit sind, eine längere Behandlungsdauer in Kauf zu nehmen (Bauer und Diedrich 1990; Hirschfeld et al. 2019). Die Kosten für eine kieferorthopädische Therapie sind für einen Großteil der Patienten ebenfalls nicht akzeptabel und somit ein Ausschlussgrund. Dessen ungeachtet haben Patienten mit definitivem Behandlungsinteresse keine Abneigung gegen eine Behandlung mit sichtbaren kieferorthopädischen Apparaturen (Hirschfeld et al. 2019).

In der vorliegenden Studie wurden die Erwartungen an eine kieferorthopädische Therapie näher betrachtet. Die Erwartungen der Patienten sind gleichzeitig die Motivationsgründe, sich durch den Kieferorthopäden behandeln zu lassen. Auffällig war, dass Patienten mit zunehmender Schwere der Parodontitis häufiger den Erhalt der eigenen Zähne im Vordergrund sahen. Die ästhetische Komponente bei einer Korrektur der Zahnstellung war für dieses Patientenkollektiv dagegen von geringerer Bedeutung. Mit Zunahme der parodontalen Destruktion stiegen aber auch hier die Erwartungen, dass die kieferorthopädische Therapie einen ästhetischen Mehrwert mit sich bringt. Gleichwohl besitzt der Erhalt der eigenen Zähne für Patienten mit einer Parodontitis eine deutlich höhere Priorität. Die in der Literatur angegeben Beweggründe für eine kieferorthopädische Behandlung sind aber sehr häufig ästhetischer Herkunft (Ehmer 1981; Bauer und Diedrich 1990; Pabari et al. 2011; Lin et al. 2016). In den Motivationsstudien mit erwachsenen Patienten im Hinblick auf eine kieferorthopädische Therapie unterlag der Wunsch nach dauerhaftem Erhalt der eigenen Zähne und funktionellen Rehabilitationen dem der Ästhetik und der Verbesserung des äußeren Erscheinungsbildes. In den letzten Jahren 
nahmen die Motivationsgründe der funktionellen Verbesserung und des dauerhaften Erhaltes der eigenen Zähne jedoch zu (Ehmer 1981; Bauer und Diedrich 1990; Pabari et al. 2011). Die in der Literatur beschriebenen Erkenntnisse zeigen also andere Motivationsgründe und Erwartungen an die Kieferorthopädie als in der vorliegenden Studie. Dies könnte in dieser Studie durch ein älteres und durch das Recallprogramm besser aufgeklärtes und motiviertes Patientenkollektiv begründet sein. Die ästhetischen Beweggründe verloren in der Studie von Bauer und Diedrich (1990) mit dem Alter an Bedeutung. Zudem wurde in der vorliegenden Studie zwischen parodontalen Erkrankungsgraden unterschieden, und Patienten mit stark parodontal kompromittierter Dentition nahmen teil. Diese Patienten zeigten, wie zuvor beschrieben, einen Zuwachs des Bewusstseins für Zähne, Zahn- und Mundgesundheit. Sie waren sich über den Verlust der Zähne durch parodontale Destruktion bewusst und sahen möglicherweise in der Kieferorthopädie eine Möglichkeit, ihre eigenen Zähne länger erhalten zu können. Die ästhetischen Komponenten verlieren zunehmend an Bedeutung, wenn Patienten an stark eingeschränkter Funktionalität leiden.

Kombiniert man die Interessen und Erwartungen der Patienten mit den klinischen Befunden und der Behandlungsbedürftigkeit, tritt eine Disharmonie auf. Um ein allumfassendes therapeutisches Konzept zu generieren, muss die Kommunikation zwischen den verschiedenen Fachdisziplinen der Zahnmedizin in Zukunft besser gewährleistet sein (Mavreas und Athanasiou 2009; Bills 2013; Acharya und Victor 2015).

Die mundgesundheitsbezogene Lebensqualität, die mit Hilfe des OHIP-G 14 und GOHAl ermittelt wurde, geht stark mit den klinischen Befunden der Patienten einher. $\mathrm{Zu}$ gleichen Ergebnissen kam eine Studie, die die mundgesundheitsbezogene Lebensqualität in Abhängigkeit vom prothetischen Versorgungsumfang ermittelte. Je größer der Zahnersatz gestaltet war, desto geringer wurde die Lebensqualität beurteilt (John et al. 2004). Nimmt der parodontale Erkrankungsgrad zu, vermehren sich die orofazialen Dysfunktionen und die Malokklusion wird gravierender. Die schwer an einer Parodontitis erkrankten Patienten beurteilten ihr Leben im Allgemeinen weniger zufriedenstellend. Hierbei wurde im Speziellen angeführt, dass es schwierig war, sich zu entspannen, Mahlzeiten in der Öffentlichkeit unterbrochen werden 
mussten und Schmerzen im Mundbereich auftraten. Die Patienten waren mit der Ästhetik der Dentition unzufrieden und sorgten sich wegen des möglichen Zahnverlustes durch die Parodontitis. Pathologische Stellungsänderungen der Zähne, Schmerzen im Mundbereich und eine eingeschränkte Funktionalität bringen häufig auch psychische Beeinträchtigungen mit sich. Betroffene Patienten vermeiden die Teilnahme am sozialen Leben und weichen einem Lächeln aus. In einer Studie von Sergl (1990) empfanden mehr als die Hälfte der befragten Personen Probleme mit den Zähnen, z.B. Zahnfehlstellungen, als störend. Außerdem gaben einige Probanden an, unter dem Aussehen gelitten zu haben und in sozialen Beziehungen eingeschränkt zu sein. Bestimmte Fehlstellungen, insbesondere auffällige Malokklusionsbefunde und lückige Zähne, können das Körperbild und Selbstbewusstsein nicht nur im Jugendalter, sondern auch im Erwachsenenalter beeinträchtigen (Helm et al. 1985; Klages et al. 2004; Sun et al. 2017). In der vorliegenden Studie konnte gezeigt werden, dass das Interesse an einer kieferorthopädischen Behandlung umso größer war, je geringer die mundgesundheitsbezogene Lebensqualität war. Ebenfalls stieg die Behandlungsbedürftigkeit aus kieferorthopädischer Sicht, beziehungsweise nahmen die Parameter der Malokklusion zu. Die kieferorthopädische Behandlung kann eine Steigerung der Zufriedenheit mit der eigenen dentofazialen Ästhetik zur Folge haben und somit psychologischen Einfluss nehmen. Aber auch eine funktionelle Rehabilitation ist das Ergebnis (Demasure-Trockels et al. 1995; Gkantidis et al. 2010; Johal et al. 2015). Patienten mit solch schwerwiegenden kieferorthopädischen und parodontologischen Befunden weisen vielschichtige Probleme auf. Sie müssen von dem Behandler aufgeklärt und für ihre Erkrankung sensibilisiert werden. Ein gemeinsames Therapiekonzept sollte Ziel der Behandlung sein. 


\subsection{Schlussfolgerung}

Im Rahmen dieser Studie konnte gezeigt werden, dass das Auftreten pathologischer Zahnwanderungen mit dem Schweregrad der parodontalen Erkrankung einhergeht. Patienten, die an einer schweren Parodontitis erkrankt waren, zeigten deutlich häufiger schwerwiegende Malokklusionen und einen großen kieferorthopädischen Behandlungsbedarf als solche, die an keiner oder moderaten Parodontitis erkrankt waren.

Das Interesse an einer kieferorthopädischen Therapie stieg mit dem Erkrankungsgrad und dementsprechend mit dem Grad der durch Zahnmigrationen bedingten Zahnfehlstellungen. Patienten mit und ohne parodontale Erkrankung legten besonderen Wert auf den Erhalt der eigenen Zähne. Von geringerer Bedeutung war die ästhetische Komponente bei einer kieferorthopädischen Therapie. Es konnte gezeigt werden, dass alle Patienten nur mangelhaft über Möglichkeiten der kieferorthopädischen Therapie aufgeklärt waren.

Die mundgesundheitsbezogene Lebensqualität war deutlich niedriger bei Patienten mit einer schweren parodontalen Erkrankung. Das Leben dieser Patienten war im Allgemeinen weniger zufriedenstellend. Sie sind eingeschränkt in der Funktionalität des stomatognathen Systems, im sozialen Leben und bei dem Versuch zu entspannen.

Die Ergebnisse bestätigen die Beobachtungen vorangegangener Studien, dass eine Parodontitis pathologische Zahnwanderungen zur Folge haben kann und Patienten in ihrer mundgesundheitsbezogenen Lebensqualität eingeschränkt sind (Brunsvold 2005; Bernhardt et al. 2019; Hirschfeld et al. 2019).

Ein dreidimensionales virtuelles Patientenmodell konnte generiert werden. Ziel zukünftiger Studien sollte es sein, ein prä- und posttherapeutisches virtuelles Patientenmodell zu erstellen, damit therapeutische Erfolge quantifiziert werden können. Der Einfluss der eingeschränkten dentofazialen Ästhetik von Patienten mit einer schweren Parodontitis auf die Wahrnehmung anderer könnte weiteren Aufschluss über soziale Aspekte liefern.

Die Erkenntnisse der vorliegenden Studie unterstreichen die vielschichtigen Probleme von Patienten mit einer schweren Parodontitis. Die Generierung eines 
interdisziplinären therapeutischen Konzepts sollte Ziel der unterschiedlichen Fachrichtungen in der Zahnmedizin sein, damit für Patienten mit derart starken Einschränkungen in Funktion, Ästhetik und sozialem Leben frühzeitig ein optimales Behandlungskonzept erstellt werden kann. 


\section{Zusammenfassung}

Ziel der Studie: Ziel dieser Untersuchung war es, pathologische Zahnwanderungen zu quantifizieren und Zusammenhänge zwischen dem Schweregrad der Parodontitis und Zahnmigrationen zu ermitteln. Bezüge zwischen der mundgesundheitsbezogenen Lebensqualität und der Parodontitis sollten hergestellt werden. Außerdem war es Ziel ein kieferorthopädisches Behandlungsinteresse und die Erwartungen an eine kieferorthopädische Behandlung aufzudecken. Ein virtuelles Patientenmodell sollte Weichteilveränderungen als Folge pathologischer Zahnwanderungen identifizieren.

Material und Methoden: Im Rahmen der vorliegenden explorativen Querschnittstudie wurden klinische, modellbezogene und dreidimensionale Daten von parodontal gesunden Patienten $(n=39)$, Patienten mit einer moderaten Parodontitis $(n=39)$ und solchen mit einer schweren parodontalen Erkrankung $(n=40)$ erfasst. Es wurden parodontale Parameter aller Patienten erhoben (ST, RZ, CAL, Lockerungsgrad). Die orthodontischen Parameter (overjet, overbite, Little-Index) wurden anhand der Gipsmodelle erhoben. Der index of treatment need wurde hinsichtlich der dentalen Gesundheit erfasst. Die Erstellung des virtuellen Patientenmodells erfolgte mit dem digitalisierten Gipsmodell und Oberflächenweichteilscans. Anschließend fand eine Vermessung des dreidimensionalen Datensatzes statt. Die Patienten gaben anhand verschiedener Fragebögen (OHIP-G 14, GOHAI) ihre mundgesundheitsbezogene Lebensqualität an. Ein individueller kieferorthopädischer Fragebogen ermöglichte es, ein Behandlungsinteresse und die Erwartungen an einer kieferorthopädischen Therapie zu quantifizieren. 
Ergebnisse: Der Malokklusionsgrad nahm mit der Schwere der parodontalen Erkrankung signifikant zu. Der kieferorthopädische Behandlungsbedarf bei Patienten mit einer schweren Form der Parodontitis wurde in $50 \%$ der Fälle groß bis sehr groß eingestuft. Die mundgesundheitsbezogene Lebensqualität dieser Patienten war eingeschränkt. Das Interesse an einer kieferorthopädischen Behandlung bei den Patienten der Studie war gering. Es wurden deutlich höhere Erwartungen an den Erhalt der eigenen Zähne als an eine Verbesserung der Ästhetik gestellt. Ein virtuelles Patientenmodell konnte der klinischen Situation entsprechend richtig erstellt werden. Die Weichteilveränderungen spiegelten nicht den Grad der parodontalen Destruktion wider.

Schlussfolgerungen: Patienten mit einer schweren parodontalen Erkrankung zeigen deutlich häufiger pathologische Zahnwanderungen und einen erhöhten kieferorthopädischen Behandlungsbedarf. Sowohl das Leben dieser Patienten im Allgemeinen als auch im Speziellen die mundgesundheitsbezogene Lebensqualität zeigen sich vermindert. Es besteht ein geringes Interesse an einer kieferorthopädischen Behandlung. Ursächlich dafür ist unter anderem die unzureichende Aufklärung über Therapieoptionen. 


\section{Anhang}

\subsection{Befundbogen}

\section{UNIVERSITÄTSMEDIZIN " $\mathbf{U} \mathbf{M G}$
GÖTTINGEN}

Prof. Dr. med. dent. P. Meyer-Marcotty

Universitätsmedizin Göttingen, Zentrum Zahn-, Mund- und Kieferheilkunde Poliklinik für Kieferorthopädie Robert-Koch-Str. 40, 37075 Göttingen; Tel.: 0551-3922366

Befundbogen

„Pathologische Zahnwanderung der Frontzähne im parodontal geschädigten Gebiss“

Patientenidentifikationsnummer:

Patientengruppe

Männlich Weiblich I II III

\section{Klinische Analyse}

Clinical Attachment Loss

\begin{tabular}{llll}
\hline \multicolumn{1}{c}{ CAL } & PD \\
\hline Starke Parodontitis & $\begin{array}{l} \pm 2 \text { interproximale Seiten } \\
\text { mit CAL } \pm \text { 6mm (nicht am } \\
\text { gleichen Zahn) }\end{array}$ & und & $\begin{array}{l} \pm 1 \text { interproximal Seite } \\
\text { mit PD } \pm 5 \mathrm{~mm}\end{array}$ \\
Moderate Parodontitis & $\begin{array}{l} \pm 2 \text { interproximale Seiten } \\
\text { mit CAL } \pm \text { 4mm (nicht am } \\
\text { gleichen Zahn) }\end{array}$ & oder & $\begin{array}{l} \pm 2 \text { interproximale Seiten } \\
\text { mit PD } \pm 5 \mathrm{~mm}\end{array}$ \\
$\begin{array}{l}\text { Keine oder Schwache } \\
\text { Parodontitis }\end{array}$ & & \\
Moderate Parodontitis & & \\
\hline
\end{tabular}

$\underline{B O P}$
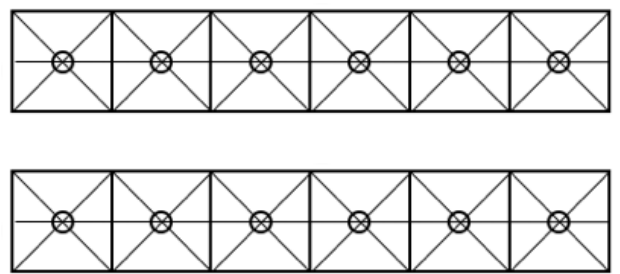

Rezessionen
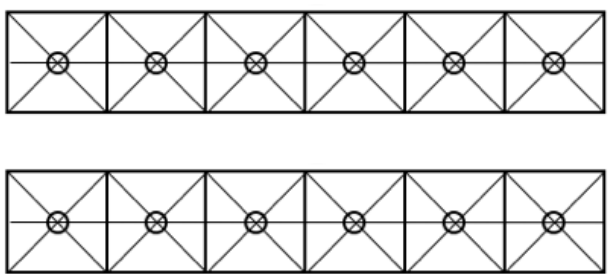

Universitătsmedizin Göttingen, Georg-August-Universität Stiftung Öffentlichen Rechts Vorstand Prof. Dr. Heyo K. Kroemer (Forschung und Lehre, Sprecher des Vorstands) Dr. Martin Siess (Krankenversorgung) Dr. Sebastian Freytag (Wirtschaftsführung und Administration) Sparkasse Göttingen (26050001) Kto: 448, IBAN: DE55 2605000100000004 48, BIC: NOLADE21GOE

Abbildung A1: Befundbogen Blatt 1 


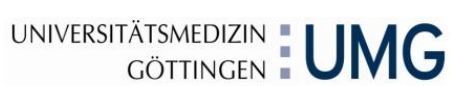 \\ Prof. Dr. med. dent. P. Meyer-Marcotty Universitätsmedizin Göttingen, Zentrum Zahn-, Mund- und Kieferheilkunde Poliklinik für Kieferorthopädie Robert-Koch-Str. 40, 37075 Göttingen; Tel.: 0551-3922366}

Patientenidentifikationsnummer:

$\underline{\text { SBI }}$
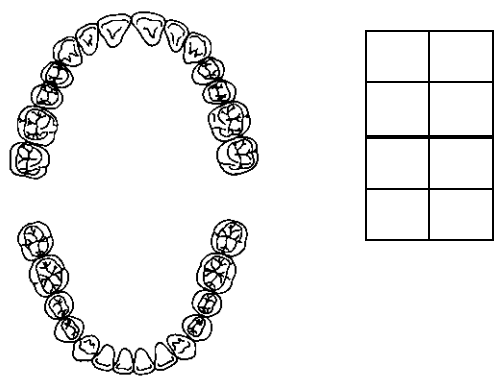

Anzahl der Positiven Messungen:

\section{Lockerungsgrad}

Grad I: bis $1 \mathrm{~mm}$

Grad II: >1mm

Grad III: beweglich auf Lippen-/ Zungengrund

\section{Gingivabiotyp}

Messstelle 11

Dick

Dünn
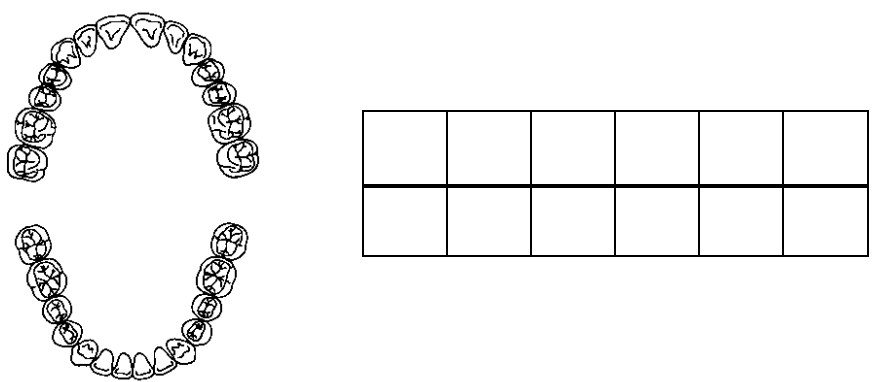

Universitătsmedizin Göttingen, Georg-August-Universităt Stiftung Öffentlichen Rechts Vorstand Prof. Dr. Heyo K. Kroemer (Forschung und Lehre, Sprecher des Vorstands) Dr. Martin Siess (Krankenversorgung) Dr. Sebastian Freytag (Wirtschaftsführung und Administration Sparkasse Göttingen (260 500 01) Kto: 448, IBAN: DE55 2605000100000004 48, BIC: NOLADE21GOE

Abbildung A2: Befundbogen Blatt 2 


\section{UNIVERSITÄTSMEDIZIN : $\mathbf{U M G}$
GÖTTINGEN :}

Prof. Dr. med. dent. P. Meyer-Marcotty Universitätsmedizin Göttingen, Zentrum Zahn-, Mund- und Kieferheilkunde Poliklinik für Kieferorthopädie Robert-Koch-Str. 40, 37075 Göttingen; Tel.: 0551-3922366

Patientenidentifikationsnummer:

\section{Modellanalyse}

\section{Overjet/Overbite in $\mathrm{mm}$}
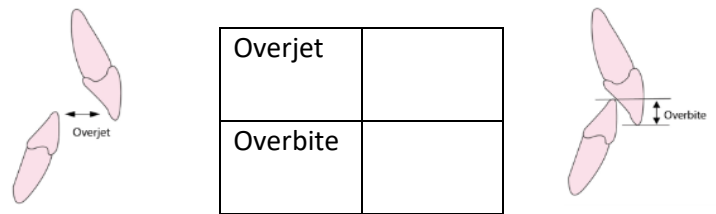

Irregularitätsindex nach Little

\begin{tabular}{|l|l|l|l|l|l|l|l|l|l|l|l|}
\hline Zahn & $43 / 42$ & $42 / 41$ & $41 / 31$ & $31 / 32$ & $32 / 33$ & Zahn & $13 / 12$ & $12 / 11$ & $11 / 21$ & $21 / 22$ & $22 / 23$ \\
\hline $\begin{array}{l}\text { Abstand } \\
\text { Kontaktpunkte }\end{array}$ & & & & & & $\begin{array}{l}\text { Abstand } \\
\text { Kontaktpunkte }\end{array}$ & & & & & \\
\hline
\end{tabular}

Summe:

Summe:

\section{Index of Treatment Need (IOTN)}

$D H C$

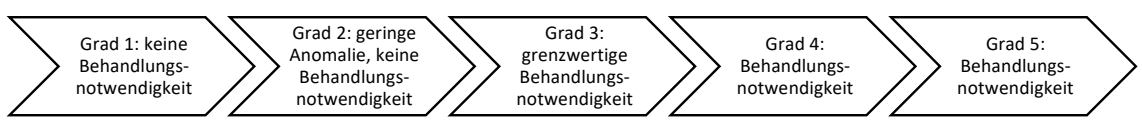
Universitätsmedizin Göttingen, Georg-August-Universität Stiftung Öffentlichen Rechts Vorstand Prof. Dr. Heyo K. Kroemer (Forschung
und Lehre, Sprecher des Vorstands) Dr. Martin Siess (Krankenversorgung) Dr. Sebastian Freytag (Wirtschaftsführung und Administration) Sparkasse Göttingen (260 500 01) Kto: 448, IBAN: DE55 2605000100000004 48, BIC: NOLADE21GOE

Abbildung A3: Befundbogen Blatt 3 


\subsection{Fragebögen}

\section{UNIVERSITÄTSMEDIZIN "
GÖTTINGEN " $\mathbf{U M G}$}

Prof Dr. med dent. P. Meyer-Marcotty Universitätsmedizin Göttingen, Zentrum Zahn-, Mund- und Kieferheilkunde Poliklinik für Kieferorthopädie

\begin{tabular}{|c|c|}
\hline Patientenindividueller kieferorthopädischer Behandlungswunsch \\
\hline
\end{tabular}

Patientenidentifikationsnummer:

\begin{tabular}{|c|c|}
\hline Alter & ............. Jahre \\
\hline Geschlecht & $\square$ männlich $\quad \square$ weiblich \\
\hline Zigarettenkonsum & ............ Zigaretten pro Tag \\
\hline $\begin{array}{l}\text { Wurde bei Ihnen Bereits eine Kieferorthopädische Behandlung } \\
\text { durchgeführt? }\end{array}$ & ............. Alter zu Beginn der Behandlung \\
\hline $\begin{array}{l}\text { Hat sich Ihr Bewusstsein für Ihre Zähne, Mund, Mundgesundheit seit } \\
\text { der parodontologischen Therapie verändert? }\end{array}$ & $\begin{array}{ll}\square & \text { sehr deutlich } \\
\square & \text { deutlich } \\
\square & \text { gering } \\
\square & \text { Gar nicht }\end{array}$ \\
\hline $\begin{array}{l}\text { Wurden Sie bereits über eine kieferorthopädische } \\
\text { Behandlungsmöglichkeit informiert? } \\
\text { (Mehrfachankreuzen möglich) }\end{array}$ & $\begin{array}{ll}\square & \text { Noch nie } \\
\square & \text { Durch den Zahnarzt } \\
\square & \text { Durch einen Kieferorthopäden } \\
\square & \text { Durch eigene Kinder } \\
\square & \text { Durch Bekannte/ Verwandte } \\
\square & \text { Durch Medien/Internet }\end{array}$ \\
\hline
\end{tabular}

\begin{tabular}{lcc}
\hline Haben Sie derzeit ein Interesse sich kieferorthopädisch behandeln zu lassen? & ja & nein \\
.. zur funktionellen Verbesserung (Kaufunktion, Lippenschluss) & $\square$ & $\square$ \\
.. wegen gesundheitlicher Aspekte (z.B. Schmerzlinderung im Kiefergelenk) & $\square$ \\
.. aus ästhetischen Gründen & $\square$ & $\square$ \\
.. für einen langfristigen Erhalt der eigenen Zähne & $\square$ & $\square$ \\
.. zur Steigerung des eigenen Wohlbefindens & $\square$ & $\square$ \\
\hline
\end{tabular}

\begin{tabular}{|c|c|c|c|}
\hline Welche Erwartungen haben/ hätten Sie an eine kieferorthopädische Therapie? & sehr & mittel & wenig \\
\hline Funktionelle Verbesserung (Kaufunktion, Lippenschluss) & $\square$ & $\square$ & $\square$ \\
\hline Gesundheitliche Aspekte (z.B. Schmerzlinderung im Kiefergelenk) & $\square$ & $\square$ & $\square$ \\
\hline Aus ästhetischen Gründen & $\square$ & $\square$ & $\square$ \\
\hline Dauerhafter Erhalt der eigenen Zähne & $\square$ & $\square$ & $\square$ \\
\hline
\end{tabular}

\footnotetext{
Universitätsmedizin Göttingen, Georg-August-Universităt Stiftung Öftentlichen Rechts Vorstand Prof. Dr. Heyo K. Kroemer (Forschung und Lehre, Sprecher des Vorstands) Dr. Martin Siess (Krankenversorgung) Dr. Sebastian Freytag (Wirtschaftsfūhrung und Administration) Sparkasse Göttingen (260500 01) Kto: 448, IBAN: DE55 2605000100000004 48, BIC: NOLADE21GOE
}

Abbildung A4: Fragebogen zum patientenindividuellen kieferorthopädischen Behandlungswunsch 


\section{OHIP- G 14}

\section{- Fragebogen zur mundgesundheitsbezogenen Lebensqualität -}

Patientenidentifikationsnummer:

Bitte prüfen Sie für jede der folgenden Aussagen, wie sehr die beschriebene Situation für Sie persönlich zutrifft. Kreuzen Sie bitte für jede Aussage eine Zahl an.

\begin{tabular}{|c|c|c|c|c|c|}
\hline $\begin{array}{l}\text { Hatten Sie im vergangenen Monat aufgrund von Problemen mit } \\
\text { Ihren Zähnen, im Mundbereich oder mit Ihrem Zahnersatz ... }\end{array}$ & $\begin{array}{c}\text { sehr } \\
\text { oft }\end{array}$ & oft & $\begin{array}{c}\text { ab } \\
\text { und } \\
\text { zu }\end{array}$ & kaum & nie \\
\hline Schwierigkeiten bestimmte Worte auszusprechen? ....................................... & 4 & 3 & 2 & 1 & $\mathbf{0}$ \\
\hline das Gefühl, Ihr Geschmackssinn war Beeinträchtigt? ..................................... & 4 & 3 & 2 & 1 & $\mathbf{0}$ \\
\hline 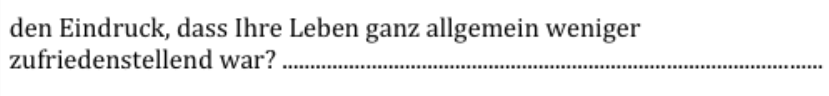 & 4 & 3 & 2 & 1 & $\mathbf{0}$ \\
\hline 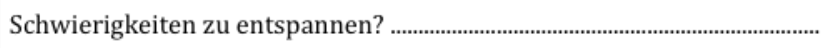 & 4 & 3 & 2 & 1 & 0 \\
\hline
\end{tabular}

Ist es im vergangenen Monat aufgrund von Problemen mit Ihren Zähnen, im Mundbereich oder mit Ihrem Zahnersatz

vorgekommen, ...

dass Sie sich angespannt gefühlt haben?

dass Sie Ihre Mahlzeiten unterbrechen mussten?

dass Sie anderen Menschen gegenüber eher reizbar gewesen sind? ......

dass es Ihnen schwergefallen ist, Ihren alltäglichen Beschäftigungen nachzugehen?

dass Sie vollkommen unfähig waren, etwas zu tun?

dass Sie sich ein wenig verlegen gefühlt haben?

dass Ihre Ernährung unbefriedigend gewesen ist?

$\begin{array}{ccccc}\begin{array}{c}\text { sehr } \\ \text { oft }\end{array} & \text { oft } & \begin{array}{c}\text { ab } \\ \text { und } \\ \text { zu }\end{array} & \text { kaum } & \text { nie } \\ 4 & 3 & 2 & 1 & 0 \\ 4 & 3 & 2 & 1 & 0 \\ 4 & 3 & 2 & 1 & 0 \\ 4 & 3 & 2 & 1 & 0 \\ 4 & 3 & 2 & 1 & 0 \\ 4 & 3 & 2 & 1 & 0 \\ 4 & 3 & 2 & 1 & 0 \\ 4 & 3 & 2 & 1 & 0\end{array}$

Hatten Sie im vergangenen Monat ...

$\begin{array}{ccccc}\begin{array}{c}\text { sehr } \\ \text { oft }\end{array} & \text { oft } & \begin{array}{c}\text { ab } \\ \text { und } \\ \text { zu }\end{array} & \text { kaum } & \text { nie } \\ 4 & 3 & 2 & 1 & 0 \\ 4 & 3 & 2 & 1 & 0\end{array}$

ein Gefühl der Unsicherheit in Zusammenhang mit Ihren Zähnen,

Ihrem Mund oder Ihrem Zahnersatz?

Universitätsmedizin Göttingen, Georg-August-Universität Stiftung Offentlichen Rechts Vorstand Prof. Dr. Heyo K. Kroemer (Forschung und Lehre, Sprecher des Vorstands) Dr. Martin Siess (Krankenversorgung) Dr. Sebastian Freytag (Wirtschaftsführung und Administration) Sparkasse Gōttingen (260 500 01) Kto: 448, IBAN: DE55 2605000100000004 48, BIC: NOLADE21GOE

Abbildung A5: Fragebogen oral health impact profile 


\section{UNIVERSITÄTSMEDIZIN "
GÖTTINGEN " $\mathbf{U M G}$}

Prof. Dr. med. dent. P. Meyer-Marcotty Universitätsmedizin Göttingen, Zentrum Zahn-, Mund- und Kieferheilkunde Poliklinik für Kieferorthopädie Poliklinik fur Kieferorthopadie

\section{German Version of the GOHAI - General Oral Health Assessment Index -}

Patientenidentifikationsnummer:

Bitte prüfen Sie für jede der folgenden Aussagen, wie sehr die beschriebene Situation für Sie persönlich zutrifft. Kreuzen Sie bitte für jede Aussage eine Zahl an.

Wie oft in den letzten drei Monaten...

$\begin{gathered}\text { sehr } \\ \text { oft }\end{gathered}$
$\quad \begin{gathered}\text { ab } \\ \text { und } \\ \text { zu }\end{gathered}$ selten nie

haben Sie den Genuss von Art und Menge von Nahrungsmitteln eingeschränkt aufgrund von Problemen mit Ihren Zähnen oder Ihrer Zahnprothese?

Hatten Sie Schwierigkeiten beim Beißen oder Kauen von

Nahrungsmitteln, wie zum Beispiel festem Fleisch oder Äpfeln?

Konnten Sie problemlos schlucken?

Konnten Sie wegen Ihrer Zähne oder Ihrer Zahnprothese nicht so sprechen wie Sie wollten?

Konnten sie ohne Beschwerden alles essen?

Haben Sie den Kontakt zu anderen Personen gemieden wegen des Zustands Ihrer Zähne oder Ihrer Zahnprothese?

Waren Sie zufrieden oder glücklich mit dem Aussehen Ihrer Zähne, des Zahnfleisches oder der Zahnprothese?

Haben Sie Medikamente genommen, um Schmerzen oder

Beschwerden im Mundbereich zu lindern?

Haben Sie sich Sorgen um Ihre Zähne, Ihr Zahnfleisch oder Ihre

Zahnprothese gemacht?

Waren Sie nervös oder unsicher, weil sie Probleme mit Ihren Zähnen, Ihrem Zahnfleisch oder Ihrer Zahnprothese hatten?

Fühlten Sie sich in Gegenwart anderer Personen beim Essen unwohl aufgrund von Problemen mit Ihren Zähnen oder Ihrer Zahnprothese?..

Reagierten Ihre Zähne oder Ihr Zahnfleisch auf heiß, kalt oder süß empfindlich?

\begin{tabular}{|c|c|c|c|c|}
\hline 4 & 3 & 2 & 1 & 0 \\
\hline 4 & 3 & 2 & 1 & 0 \\
\hline 4 & 3 & 2 & 1 & 0 \\
\hline 4 & 3 & 2 & 1 & 0 \\
\hline 4 & 3 & 2 & 1 & 0 \\
\hline 4 & 3 & 2 & 1 & 0 \\
\hline 4 & 3 & 2 & 1 & 0 \\
\hline 4 & 3 & 2 & 1 & 0 \\
\hline 4 & 3 & 2 & 1 & 0 \\
\hline 4 & 3 & 2 & 1 & 0 \\
\hline 4 & 3 & 2 & 1 & 0 \\
\hline 4 & 3 & 2 & 1 & 0 \\
\hline
\end{tabular}

Universitätsmedizin Göttingen, Georg-August-Universitāt Stiftung Offentlichen Rechts Vorstand Prof. Dr. Heyo K. Kroemer (Forschung und Lehre, Sprecher des Vorstands) Dr. Martin Siess (Krankenversorgung) Dr. Sebastian Frevtag (Wirtschaftsführung und Administration) Sparkasse Göttingen (260500 01) Kto: 448, IBAN: DE55 2605000100000004 48, BIC: NOLADE21GOE

Abbildung A6: Fragebogen general / geriatric oral health assessment index 


\subsection{Parameter der Gruppeneinteilung}
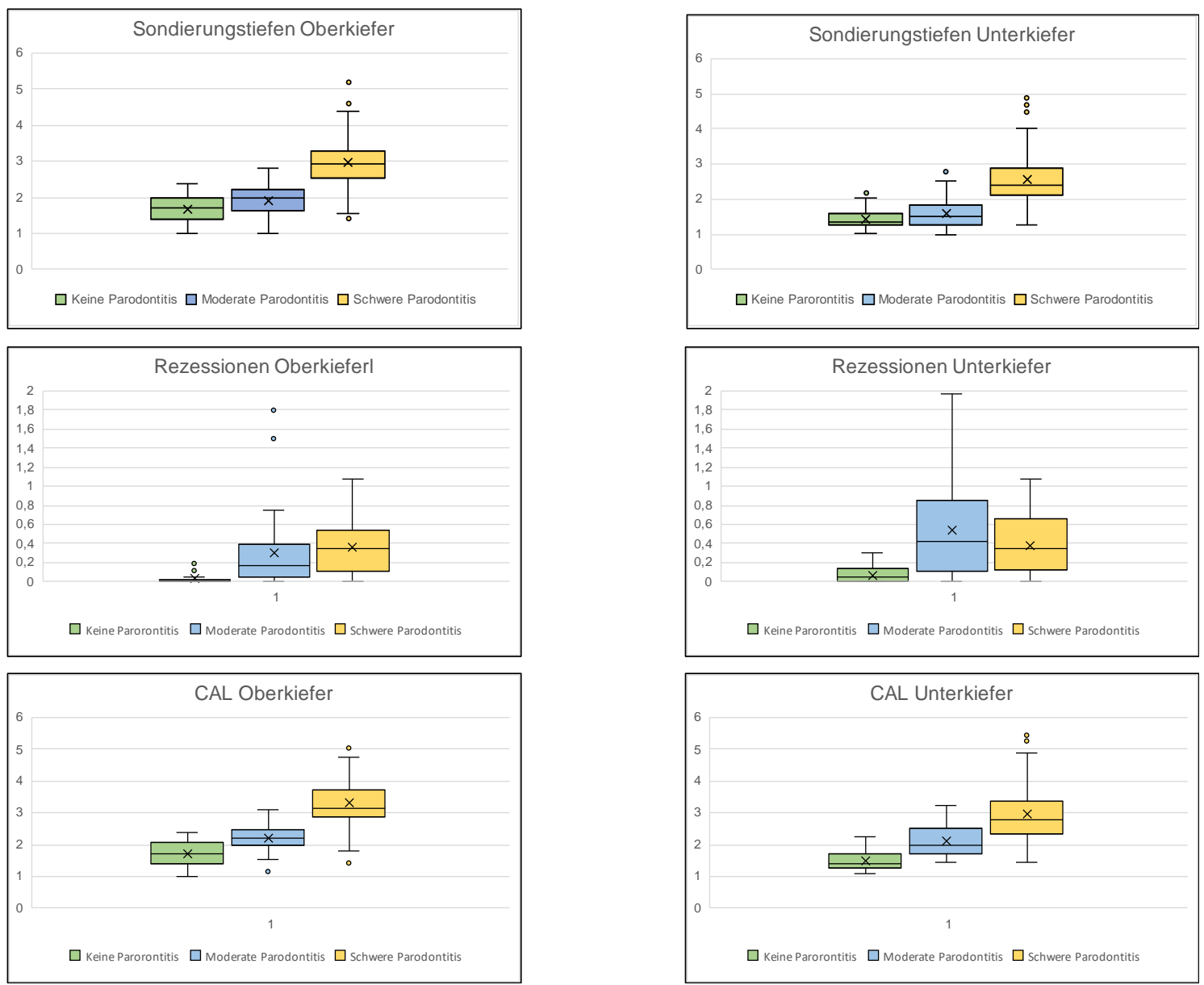

Abbildung A7: Box Plot Darstellungen zu den Ergebnissen der Parameter der Gruppeneinteilung 


\section{Literaturverzeichnis}

Acharya V, Victor D (2015): Orthodontic management of patients undergoing prosthetic rehabilitation. J Calif Dent Assoc 느, 185-191

Albandar JM (2005): Epidemiology and risk factors of periodontal diseases. Dent Clin North Am $\underline{49}$, 517-532

Albandar JM (2014): Aggressive periodontitis: case definition and diagnostic criteria. Periodontol $2000 \underline{65}, 13-26$

Armitage GC (1996): Periodontal diseases: diagnosis. Ann Periodontol 1, 37-215

Armitage GC (1999): Development of a classification system for periodontal diseases and conditions. Ann Periodontol $\underline{4}, 1-6$

Atchison KA, Dolan TA (1990): Development of the Geriatric Oral Health Assessment Index. J Dent Educ 54, 680-687

Ayoub AF, Wray D, Moos KF, Siebert P, Jin J, Niblett TB, Urquhart C, Mowforth R (1996): Three-dimensional modeling for modern diagnosis and planning in maxillofacial surgery. Int J Adult Orthodon Orthognath Surg 11, 225-233

Bascones-Martinez A, Figuero-Ruiz E (2004): Periodontal diseases as bacterial infection. Med Oral Patol Oral Cir Bucal (Supp 9), 92-107

Bauer W, Diedrich P (1990): Motivation und Erfolgsbeurteilung erwachsener Patienten zur kieferorthopädischen Behandlung - Interpretation einer Befragung. Fortschr Kieferorthop $\underline{51}$, 180-188

Beck JD, Koch GG, Rozier RG, Tudor GE (1990): Prevalence and risk indicators for periodontal attachment loss in a population of older community-dwelling blacks and whites. J Periodontol $\underline{61}, 521-528$

Berglundh T, Armitage G, Araujo MG, Avila-Ortiz G, Blanco J, Camargo PM, Chen S, Cochran D, Derks J, Figuero E et al. (2018): Peri-implant diseases and conditions: Consensus report of workgroup 4 of the 2017 World Workshop on the Classification of Periodontal and Peri-Implant Diseases and Conditions. J Clin Periodontol $\underline{45}$ (Supp 20), 286-291

Bergstrom J (1989): Cigarette smoking as risk factor in chronic periodontal disease. Community Dent Oral Epidemiol 17, 245-247

Bernhardt O, Krey KF, Daboul A, Volzke H, Kindler S, Kocher T, Schwahn C (2019): New insights in the link between malocclusion and periodontal disease. $J$ Clin Periodontol $\underline{46}, 144-159$

Bills D (2013): Collaboration between the general dentist and orthodontist: an essential partnership to ensure orthodontic success. Compend Contin Educ Dent 34, 262-267 
Borges CM, Peres MA, Peres KG (2010): Association between malocclusion and dissatisfaction with dental and gingival appearance: study with Brazilian adolescents. Rev Bras Epidemiol 13, 713-723

Brook PH, Shaw WC (1989): The development of an index of orthodontic treatment priority. Eur J Orthod 11, 309-320

Brunsvold MA (2005): Pathologic Tooth Migration. J Periodontol $\underline{76}$, 859-866

Caffesse RG (1980): Management of periodontal disease in patients with occlusal abnormalities. Dent Clin North Am 24, 215-230

Camara CA, Martins RP (2016): Functional Aesthetic Occlusal Plane (FAOP). Dental Press J Orthod 21, 114-125

Cao T, Xu L, Shi J, Zhou Y (2015): Combined orthodontic-periodontal treatment in periodontal patients with anteriorly displaced incisors. Am J Orthod Dentofacial Orthop $\underline{148}, 805-813$

Cardaropoli D, Re S, Corrente G, Abundo R (2001): Intrusion of migrated incisors with infrabony defects in adult periodontal patients. Am J Orthod Dentofacial Orthop $\underline{120}, 671-675$

Carvalho CV, Saraiva L, Bauer FPF, Kimura RY, Souto MLS, Bernardo CC, Pannuti CM, Romito GA, Pustiglioni FE (2018): Orthodontic treatment in patients with aggressive periodontitis. Am J Orthod Dentofacial Orthop 153, 550-557

Caton JG, Armitage G, Berglundh T, Chapple ILC, Jepsen S, Kornman KS, Mealey BL, Papapanou PN, Sanz M, Tonetti MS (2018): A new classification scheme for periodontal and peri-implant diseases and conditions - Introduction and key changes from the 1999 classification. J Clin Periodontol $\underline{45}, 1-8$

Ceinos R, Lupi L, Tellier A, Bertrand MF (2017): Three-dimensional stereophotogrammetric analysis of 50 smiles: A study of dento-facial proportions. J Esthet Restor Dent $\underline{00,1-8}$

Celebi AA, Kau CH, Ozaydin B (2017): Three-Dimensional Anthropometric Evaluation of Facial Morphology. J Craniofac Surg 28, 470-474

Chapple ILC, Mealey BL, Van Dyke TE, Bartold PM, Dommisch H, Eickholz P, Geisinger ML, Genco RJ, Glogauer M, Goldstein M et al. (2018): Periodontal health and gingival diseases and conditions on an intact and a reduced periodontium: Consensus report of workgroup 1 of the 2017 World Workshop on the Classification of Periodontal and Peri-Implant Diseases and Conditions. $J$ Periodontol $\underline{89}$ (Supp 1), 74-84

Chasens Al (1979): Periodontal disease, pathologic tooth migration and adult orthodontics. N Y J Dent $\underline{49}$, 40-43

Cho MI, Garant PR (2000): Development and general structure of the periodontium. Periodontol $2000 \underline{24,}$ 9-27 
Cirelli JA, Cirelli CC, Holzhausen M, Martins LP, Brandao CH (2006): Combined periodontal, orthodontic, and restorative treatment of pathologic migration of anterior teeth: a case report. Int J Periodontics Restorative Dent 26, 501-506

Clarke NG, Hirsch RS (1995): Personal risk factors for generalized periodontitis. J Clin Periodontol 22, 136-145

de Couto Nascimento V, de Castro Ferreira Conti AC, de Almeida Cardoso M, Valarelli DP, de Almeida-Pedrin RR (2016): Impact of orthodontic treatment on self-esteem and quality of life of adult patients requiring oral rehabilitation. Angle Orthod $\underline{86}, 839-845$

de Luca Canto G, Pacheco-Pereira C, Lagravere MO, Flores-Mir C, Major PW (2015): Intra-arch dimensional measurement validity of laser-scanned digital dental models compared with the original plaster models: a systematic review. Orthod Craniofac Res $\underline{18}$, 65-76

de Pablo P, Dietrich T, McAlindon TE (2008): Association of periodontal disease and tooth loss with rheumatoid arthritis in the US population. J Rheumatol $\underline{35}, 70-$ 76

Deinzer R, Micheelis W, Granrath N, Hoffmann T (2009): More to learn about: periodontitis-related knowledge and its relationship with periodontal health behaviour. J Clin Periodontol $\underline{36}, 756-764$

Demasure-Trockels P, Katsaros C, Besold G, Berg R (1995): Psychosoziale Aspekte bei kieferorthopädisch behandelten und unbehandelten Erwachsenen mit ähnlichen, gut sichtbaren Zahnstellungsfehlern. Fortschr Kieferorthop 56, 7783

Dewhirst FE, Chen T, Izard J, Paster BJ, Tanner AC, Yu WH, Lakshmanan A, Wade WG (2010): The human oral microbiome. J Bacteriol 192, 5002-5017

Diedrich P (1989): Wechselbeziehungen zwischen Kieferorthopädie und Parodontologie. Fortschr Kieferorthop 50, 347-364

Diedrich P (1992): Die Korrektur aufgefächerter Frontzähne - ein wichtiger kieferorthopädischer Beitrag zur ästhetischen Zahnheilkunde. Fortschr Kieferorthop $\underline{53}$, 304-311

Doyle CJ, Bartold PM (2012): How does stress influence periodontitis? J Int Acad Periodontol 14, $42-49$

Duran GS, Dindaroglu F, Kutlu P (2019): Hard - and soft-tissue symmetry comparison in patients with Class III malocclusion. Am J Orthod Dentofacial Orthop $\underline{155}, 509-522$

Dvortsin DP, Ye Q, Pruim GJ, Dijkstra PU, Ren Y (2011): Reliability of the integrated radiograph-photograph method to obtain natural head position in cephalometric diagnosis. Angle Orthod 81, 889-894

Edman K, Ohrn K, Nordstrom B, Holmlund A, Hellberg D (2015): Trends over 30 years in the prevalence and severity of alveolar bone loss and the influence of 
smoking and socio-economic factors-based on epidemiological surveys in Sweden 1983-2013. Int J Dent Hyg 13, 283-291

Eger T, Muller HP, Heinecke A (1996): Ultrasonic determination of gingival thickness. Subject variation and influence of tooth type and clinical features. $J$ Clin Periodontol 23, 839-845

Ehmer U (1981): Motivation zur kieferorthopfidischen Behandlung aus der Sicht des Patienten und seiner Eltern in Beziehung zu objektiven Symptomen der Dysgnathie. Fortschr Kieferorthop $\underline{42}$, 441-450

Eke PI, Dye BA, Wei L, Thornton-Evans GO, Genco RJ (2012): Prevalence of periodontitis in adults in the United States: 2009 and 2010. J Dent Res 91, 914920

Eliasson LA, Hugoson A, Kurol J, Siwe H (1982): The effects of orthodontic treatment on periodontal tissues in patients with reduced periodontal support. Eur J Orthod $\underline{4}, 1-9$

Ericsson I (1986): The combined effects of plaque and physical stress on periodontal tissues. J Clin Periodontol $\underline{13}$, 918-922

Espeland LV, Stenvik A (1991): Perception of personal dental appearance in young adults: relationship between occlusion, awareness, and satisfaction. Am J Orthod Dentofacial Orthop 100, 234-241

Feneis H (1952): Gefüge und Funktion des normalen Zahnfleischbindegewebes. Dtsch Zahnarztl Z $\underline{7}, 467-476$

Fisk R (1960): When Malocclusion Concerns the Public. J Can Dent Assoc 26, 397-412

Galantucci LM, Deli R, Laino A, Di Gioia E, D'Alessio R, Lavecchia F, Percoco G, Savastano C (2016): Three-Dimensional Anthropometric Database of Attractive Caucasian Women: Standards and Comparisons. J Craniofac Surg 27, 18841895

Gher ME (1998): Changing concepts. The effects of occlusion on periodontitis. Dent Clin North Am 42, 285-299

Gkantidis N, Christou P, Topouzelis N (2010): The orthodontic-periodontic interrelationship in integrated treatment challenges: a systematic review. J Oral Rehabil 37, 377-390

Glavind L, Loe H (1967): Errors in the clinical assessment of periodontal destruction. J Periodontal Res 2 , 180-184

Goldstein MC (1953): Adult Orthodontics. Am J Orthod Dentofacial Orthop $\underline{39}$, 400-424

Greenstein G (1997): Contemporary interpretation of probing depth assessments: diagnostic and therapeutic implications. A literature review. J Periodontol $\underline{68}, 1194-1205$ 
Gross AJ, Paskett KT, Cheever VJ, Lipsky MS (2017): Periodontitis: a global disease and the primary care provider's role. Postgrad Med J $\underline{93}, 560-565$

Grossi SG, Genco RJ, Machtei EE, Ho AW, Koch G, Dunford R, Zambon JJ, Hausmann E (1995): Assessment of risk for periodontal disease. II. Risk indicators for alveolar bone loss. J Periodontol $\underline{66}, 23-29$

Grossi SG, Zambon JJ, Ho AW, Koch G, Dunford RG, Machtei EE, Norderyd OM, Genco RJ (1994): Assessment of risk for periodontal disease. I. Risk indicators for attachment loss. J Periodontol $\underline{65}$, 260-267

Gwilliam JR, Cunningham SJ, Hutton T (2006): Reproducibility of soft tissue landmarks on three-dimensional facial scans. Eur J Orthod $\underline{28}, 408-415$

Hassel AJ, Rolko C, Koke U, Leisen J, Rammelsberg P (2008): A German version of the GOHAI. Community Dent Oral Epidemiol $\underline{36}, 34-42$

Häupl K, Lang FJ: Die Marginale Paradentitis. Verlag von Hermann Meusser, Berlin 1927

Havemose-Poulsen A, Westergaard J, Stoltze K, Skjodt H, Danneskiold-Samsoe $\mathrm{B}$, Locht H, Bendtzen K, Holmstrup P (2006): Periodontal and hematological characteristics associated with aggressive periodontitis, juvenile idiopathic arthritis, and rheumatoid arthritis. J Periodontol $\underline{77}$, 280-288

Hefti AF (1997): Periodontal probing. Crit Rev Oral Biol Med $\underline{8}$, 336-356

Helenius LM, Hallikainen D, Helenius I, Meurman JH, Kononen M, Leirisalo-Repo M, Lindqvist C (2005): Clinical and radiographic findings of the temporomandibular joint in patients with various rheumatic diseases. A casecontrol study. Oral Surg Oral Med Oral Pathol Oral Radiol Endod 99, 455-463

Helm S, Kreiborg S, Solow B (1985): Psychosocial implications of malocclusion: a 15-year follow-up study in 30-year-old Danes. Am J Orthod 87, 110-118

Hensel E, Born G, Korber V, Altvater T, Gesch D (2003): Prevalence of defined symptoms of malocclusion among probands enrolled in the Study of Health in Pomerania (SHIP) in the age group from 20 to 49 years. J Orofac Orthop 64 166

Hirschfeld J, Reichardt E, Sharma P, Hilber A, Meyer-Marcotty P, StellzigEisenhauer A, Schlagenhauf U, Sickel FE (2019): Interest in orthodontic tooth alignment in adult patients affected by periodontitis: A questionnaire-based crosssectional pilot study. J Periodontol 90, 957-965

Hojo K, Nagaoka S, Ohshima T, Maeda N (2009): Bacterial interactions in dental biofilm development. J Dent Res $\underline{88}, 982-990$

Holtfreter B, Albandar JM, Dietrich T, Dye BA, Eaton KA, Eke PI, Papapanou PN, Kocher T (2015): Standards for reporting chronic periodontitis prevalence and severity in epidemiologic studies: Proposed standards from the Joint EU/USA Periodontal Epidemiology Working Group. J Clin Periodontol 42, 407-412 
Holtfreter B, Alte D, Schwahn C, Desvarieux M, Kocher T (2012): Effects of different manual periodontal probes on periodontal measurements. J Clin Periodontol $\underline{39}$, 1032-1041

Holtfreter B, Schwahn C, Biffar R, Kocher T (2009): Epidemiology of periodontal diseases in the Study of Health in Pomerania. J Clin Periodontol $\underline{36}, 114-123$

Horup N, Melsen B, Terp S (1987): Relationship between malocclusion and maintenance of teeth. Community Dent Oral Epidemiol 15, 74-78

Hunt O, Hepper P, Johnston C, Stevenson M, Burden D (2001): Professional perceptions of the benefits of orthodontic treatment. Eur J Orthod $\underline{23}, 315-323$

Jang KS, Bayome M, Park JH, Park KH, Moon HB, Kook YA (2017): A threedimensional photogrammetric analysis of the facial esthetics of the Miss Korea pageant contestants. Korean J Orthod ㄴ7, 87-99

Jepsen K, Jaeger A, Jepsen S (2015): Esthetic and functional rehabilitation of a severely compromised central incisor: an interdisciplinary approach. Int $\mathrm{J}$ Periodontics Restorative Dent 35, 35-43

Jepsen S, Caton JG, Albandar JM, Bissada NF, Bouchard P, Cortellini P, Demirel K, de Sanctis M, Ercoli C, Fan J et al. (2018): Periodontal manifestations of systemic diseases and developmental and acquired conditions: Consensus report of workgroup 3 of the 2017 World Workshop on the Classification of Periodontal and Peri-Implant Diseases and Conditions. J Clin Periodontol $\underline{45}$, 219-229

Johal A, Alyaqoobi I, Patel R, Cox S (2015): The impact of orthodontic treatment on quality of life and self-esteem in adult patients. Eur J Orthod 37, 233-237

John MT, Micheelis W, Biffar R (2004): Normwerte mundgesundheitsbezogener Lebensqualität für Kurzversionen des Oral Health Impact Profile. Schweiz Monatsschr Zahnmed 114, 784-791

John MT, Patrick DL, Slade GD (2002): The German version of the Oral Health Impact Profile-translation and psychometric properties. Eur J Oral Sci 110, 425433

Jordan RA, Bodechtel C, Hertrampf K, Hoffmann T, Kocher T, Nitschke I, Schiffner U, Stark H, Zimmer S, Micheelis W (2014): The Fifth German Oral Health Study (Fünfte Deutsche Mundgesundheitsstudie, DMS V) - rationale, design, and methods. BMC Oral Health $\underline{14}, 161$

Joshi M, Wu LP, Maharjan S, Regmi MR (2015): Sagittal lip positions in different skeletal malocclusions: a cephalometric analysis. Prog Orthod 16, 1-8

Joss CU, Joss-Vassalli IM, Berge SJ, Kuijpers-Jagtman AM (2010): Soft tissue profile changes after bilateral sagittal split osteotomy for mandibular setback: a systematic review. J Oral Maxillofac Surg $\underline{68}$, 2792-2801 
Kawano Y (1987): Three dimensional analysis of the face in respect of zygomatic fractures and evaluation of the surgery with the aid of Moire topography. $J$ Craniomaxillofac Surg $\underline{15}, 68-74$

Kessler M (1976): Interrelationships between orthodontics and periodontics. Am J Orthod $\underline{70}, 154-172$

Kim HH, Lee JW, Cha KS, Chung DH, Lee SM (2014): Three-dimensional assessment of upper lip positional changes according to simulated maxillary anterior tooth movements by white light scanning. Korean J Orthod 444, 281-293

Kirschneck C, Fanghanel J, Wahlmann U, Wolf M, Roldan JC, Proff P (2017): Interactive effects of periodontitis and orthodontic tooth movement on dental root resorption, tooth movement velocity and alveolar bone loss in a rat model. Ann Anat $\underline{210}, 32-43$

Klages U, Bruckner A, Zentner A (2004): Dental aesthetics, self-awareness, and oral health-related quality of life in young adults. Eur J Orthod $\underline{26}, 507-514$

Konig J, Holtfreter B, Kocher T (2010): Periodontal health in Europe: future trends based on treatment needs and the provision of periodontal services-position paper 1. Eur J Dent Educ 14, 4-24

Kuhn M, Markic G, Doulis I, Gollner P, Patcas R, Hanggi MP (2016): Effect of different incisor movements on the soft tissue profile measured in reference to a rough-surfaced palatal implant. Am J Orthod Dentofacial Orthop 149, 349-357

Landsberger DR: Der Einfluß der Zähne auf die Entwicklung des Schädels (Archiv für Anatomie und Physiologie). Verlag von Veit \& Comp., Leipzig 1911

Lange DE: Parodontologie in der täglichen Praxis. Quintessenz Verlag-GmbH, Berlin 1981

Li H, Cao T, Zhou H, Hou Y (2019): Lip position analysis of young women with different skeletal patterns during posed smiling using 3-dimensional stereophotogrammetry. Am J Orthod Dentofacial Orthop 155, 64-70

Lin F, Ren M, Yao L, He Y, Guo J, Ye Q (2016): Psychosocial impact of dental esthetics regulates motivation to seek orthodontic treatment. Am J Orthod Dentofacial Orthop 150, 476-482

Listgarten MA (1980): Periodontal probing: what does it mean? J Clin Periodontol 7, $165-176$

Little RM (1975): The irregularity index: a quantitative score of mandibular anterior alignment. Am J Orthod $\underline{68}, 554-563$

Locker D, Matear D, Stephens M, Lawrence H, Payne B (2001): Comparison of the GOHAI and OHIP-14 as measures of the oral health-related quality of life of the elderly. Community Dent Oral Epidemiol 29, 373-381

Loe H (1983): Principles of aetiology and pathogenesis governing the treatment of periodontal disease. Int Dent J $\underline{33}, 119-126$ 
Loe H, Theilade E, Jensen SB (1965): Experimental Gingivitis in Man. J Periodontol $\underline{36}, 177-187$

Martinez-Canut P, Carrasquer A, Magan R, Lorca A (1997): A study on factors associated with pathologic tooth migration. J Clin Periodontol $\underline{24}, 492-497$

Masoud MI, Bansal N, J CC, Manosudprasit A, Allareddy V, Haghi A, Hawkins HC, Otarola-Castillo E (2017): 3D dentofacial photogrammetry reference values: a novel approach to orthodontic diagnosis. Eur J Orthod $\underline{39}$, 215-225

Mavreas D, Athanasiou AE (2009): Orthodontics and its interactions with other dental disciplines. Prog Orthod 10, 72-81

McCall JO (1944): A study of malocclusion in pre-school and school children. Dental Items of Interest Pub. Co. $\underline{66}, 131-133$

McCance AM, Moss JP, Wright WR, Linney AD, James DR (1992): A threedimensional soft tissue analysis of 16 skeletal class III patients following bimaxillary surgery. Br J Oral Maxillofac Surg 30, 221-232

McKiernan EX, McKiernan F, Jones ML (1992): Psychological profiles and motives of adults seeking orthodontic treatment. Int $\mathrm{J}$ Adult Orthodon Orthognath Surg $\underline{7}, 187-198$

Melsen B, Agerbaek N, Markenstam G (1989): Intrusion of incisors in adult patients with marginal bone loss. Am J Orthod Dentofacial Orthop $\underline{96}$, 232-241

Meyle J, Chapple I (2015): Molecular aspects of the pathogenesis of periodontitis. Periodontol $2000 \underline{69}, 7-17$

Minch LE, Sarul M, Nowak R, Kawala B, Antoszewska-Smith J (2017): Orthodontic intrusion of periodontally-compromised maxillary incisors: 3dimensional finite element method analysis. Adv Clin Exp Med 르, 829-833

Moss JP, Grindrod SR, Linney AD, Arridge SR, James D (1988): A computer system for the interactive planning and prediction of maxillofacial surgery. Am J Orthod Dentofacial Orthop 94, 469-475

Müller Campanile V, Megally A, Campanile G, Gayet-Ageron A, Giannopoulou C, Mombelli A (2019): Risk factors for recurrence of periodontal disease in patients in maintenance care in a private practice. J Clin Periodontol $\underline{46}$, 918-926

Müller HP, Heinecke A, Schaller N, Eger T (2000): Masticatory mucosa in subjects with different periodontal phenotypes. J Clin Periodontol 27, 621-626

Musich DR (2004): Mythen und Missverständnisse bei Diagnostik und Behandlung von Erwachsenen. Inf Orthod Kieferorthop 36, 99-114

Nakre PD, Harikiran AG (2013): Effectiveness of oral health education programs: A systematic review. J Int Soc Prev Community Dent $\underline{3}$, 103-115

Newman MG (1985): Current concepts of the pathogenesis of periodontal disease. Microbiology emphasis. J Periodontol $\underline{56}, 734-739$ 
Ngom PI, Diagne F, Benoist HM, Thiam F (2006): Intraarch and interarch relationships of the anterior teeth and periodontal conditions. Angle Orthod $\underline{76}$, 236-242

Oh SL (2011): An interdisciplinary treatment to manage pathologic tooth migration: a clinical report. J Prosthet Dent 106, 153-158

Öhrn K, Jönsson B (2012): A comparison of two questionnaires measuring oral health-related quality of life before and after dental hygiene treatment in patients with periodontal disease. Int J Dent Hyg 10, 9-14

Ong MM, Wang HL (2002): Periodontic and orthodontic treatment in adults. Am J Orthod Dentofacial Orthop 122, 420-428

Pabari S, Moles DR, Cunningham SJ (2011): Assessment of motivation and psychological characteristics of adult orthodontic patients. Am J Orthod Dentofacial Orthop 140, 263-272

Page RC, Eke PI (2007): Case definitions for use in population-based surveillance of periodontitis. J Periodontol $\underline{78}$ (Supp 7), 1387-1399

Page RC, Kornman KS (1997): The pathogenesis of human periodontitis: an introduction. Periodontol $2000 \underline{14}, 9-11$

Palmer RM, Wilson RF, Hasan AS, Scott DA (2005): Mechanisms of action of environmental factors-tobacco smoking. J Clin Periodontol 32 (Supp 6), 180-195

Papapanou PN, Sanz M, Buduneli N, Dietrich T, Feres M, Fine DH, Flemmig TF, Garcia R, Giannobile WV, Graziani F et al. (2018): Periodontitis: Consensus report of workgroup 2 of the 2017 World Workshop on the Classification of Periodontal and Peri-Implant Diseases and Conditions. J Clin Periodontol $\underline{45}$ (Supp 20), 162-170

Papapanou PN, Susin C (2017): Periodontitis epidemiology: is periodontitis under-recognized, over-diagnosed, or both? Periodontol $2000 \underline{75}, 45-51$

Pihlstrom BL, Michalowicz BS, Johnson NW (2005): Periodontal diseases. Lancet $\underline{366}, 1809-1820$

Pischon N, Pischon T, Kroger J, Gulmez E, Kleber BM, Bernimoulin JP, Landau $\mathrm{H}$, Brinkmann PG, Schlattmann P, Zernicke J et al. (2008): Association among rheumatoid arthritis, oral hygiene, and periodontitis. J Periodontol $\underline{79}$, 979-986

Popova C, Dosseva-Panova V, Panov V (2013): Microbiology of periodontal diseases. A review. Biotechnol Biotechnol Equip 27, 3754-3759

Poulsen $\mathrm{H}$, Meurman JH, Kautiainen H, Heikkinen AM, Huvinen E, Koivusalo S, Eriksson JG (2019): Oral Health in Women with a History of High Gestational Diabetes Risk. Dent J $\underline{7}, 92$

Preber $\mathrm{H}$, Bergstrom $\mathrm{J}$ (1986): Cigarette smoking in patients referred for periodontal treatment. Scand J Dent Res $\underline{94}, 102-108$ 
Preber H, Bergstrom J (1990): Effect of cigarette smoking on periodontal healing following surgical therapy. J Clin Periodontol 17, 324-328

Proffit WR (1978): Equilibrium theory revisited: factors influencing position of the teeth. Angle Orthod $\underline{48}, 175-186$

Proffit WR, Fields HW, Jr., Moray LJ (1998): Prevalence of malocclusion and orthodontic treatment need in the United States: estimates from the NHANES III survey. Int J Adult Orthodon Orthognath Surg 13, 97-106

Ramfjord SP, Ash MM, Jr. (1981): Significance of occlusion in the etiology and treatment of early, moderate, and advanced periodontitis. J Periodontol $\underline{52}, 511$ 517

Rangel FA, Chiu YT, Maal TJ, Bronkhorst EM, Berge SJ, Kuijpers-Jagtman AM (2016): Does powdering of the dentition increase the accuracy of fusing 3D stereophotographs and digital dental casts. Eur J Orthod $\underline{38}, 440-445$

Rangel FA, Maal TJ, Berge SJ, van Vlijmen OJ, Plooij JM, Schutyser F, KuijpersJagtman AM (2008): Integration of digital dental casts in 3-dimensional facial photographs. Am J Orthod Dentofacial Orthop 134, 820-826

Rangel FA, Maal TJJ, de Koning MJJ, Bronkhorst EM, Berge SJ, KuijpersJagtman AM (2018): Integration of digital dental casts in cone beam computed tomography scans-a clinical validation study. Clin Oral Investig ⒉, 1215-1222

Rateitschak KH, Rateitschak EM, Wolf HF (Hrsg.): Farbatlanten der Zahnmedizin Parodontologie Band 1. Georg Thieme Verlag, Stuttgart 1989

Rateitschak KH, Rateitschak EM, Wolf HF (Hrsg.): Farbatlanten der Zahnmedizin Parodontologie Band 1. Georg Thieme Verlag, 2012

Re S, Cardaropoli D, Abundo R, Corrente G (2004): Reduction of gingival recession following orthodontic intrusion in periodontally compromised patients. Orthod Craniofac Res $\underline{7}, 35-39$

Reynolds MA (2014): Modifiable risk factors in periodontitis: at the intersection of aging and disease. Periodontol $2000 \underline{64}$, 7-19

Richtlinien des Bundesausschusses der Zahnärzte und Krankenkassen für die kieferorthopädische Behandlung (2003): Richtlinien des Bundesausschusses der Zahnärzte und Krankenkassen für die kieferorthopädische Behandlung in der Fassung vom 04. Juni 2003 und vom 24. September 2003 veröffentlicht im Bundesanzeiger Nr. 226 (S. 24 966) vom 03. Dezember 2003 in Kraft getreten am 1. Januar 2004

Ricketts RM (1960): A foundation for cephalometric communication. American Journal of Orthodontics $\underline{46}, 330-357$

Rosati R, De Menezes M, Rossetti A, Sforza C, Ferrario VF (2010): Digital dental cast placement in 3-dimensional, full-face reconstruction: a technical evaluation. Am J Orthod Dentofacial Orthop $\underline{138}, 84-88$ 
Rosati R, Rossetti A, De Menezes M, Ferrario VF, Sforza C (2012): The occlusal plane in the facial context: inter-operator repeatability of a new three-dimensional method. Int J Oral Sci 4, 34-37

Rossini G, Parrini S, Castroflorio T, Deregibus A, Debernardi CL (2016): Diagnostic accuracy and measurement sensitivity of digital models for orthodontic purposes: A systematic review. Am J Orthod Dentofacial Orthop $\underline{149}$, $161-170$

Rozier RG, White BA, Slade GD (2017): Trends in Oral Diseases in the U.S. Population. J Dent Educ 81, 97-109

Rutherford ST, Bassler BL (2012): Bacterial quorum sensing: its role in virulence and possibilities for its control. Cold Spring Harb Perspect Med 2, $1-25$

Sawyer AR, See M, Nduka C (2009): 3D stereophotogrammetry quantitative lip analysis. Aesthetic Plast Surg $\underline{33}$, 497-504

Schiffner U: Fünfte Deutsche Mundgesundheitsstudie (DMS V). Deutscher Zahnärtzte Verlag, Köln 2016

Schroeder HE: Handbook of Microscopic Anatomy Volume V/5: The Periodontium. Springer-Verlag, Berlin 1986

Schroeder HE: Oral Structural Biology: Embryology, Structure, and Function of Normal Hard and Soft Tissues of the Oral Cavity and Temporomandibular Joints. Georg Thieme Verlag, Stuttgart 1991

Schroeder HE: Pathobiologie oraler Strukturen. Karger Verlag, Basel 1997

Schroeder HE, Listgarten MA (1997): The gingival tissues: the architecture of periodontal protection. Periodontol $2000 \underline{13}, 91-120$

Schutzhold S, Kocher T, Biffar R, Hoffmann T, Schmidt CO, Micheelis W, Jordan $\mathrm{R}$, Holtfreter B (2015): Changes in prevalence of periodontitis in two German population-based studies. J Clin Periodontol 42, 121-130

Schwenzer K, Holberg C, Willer J, Mast G, Ehrenfeld M (1998): 3D-Erfassung der Gesichtsoberfläche durch Topometrie unter der Verwendung von projizierten Weißlichtstreifen. Mund Kiefer Gesichtschir 2 (Supp 1), 130-134

Selvig KA, Mjör IA: Histology of the Human Tooth. Munksgaard, Kopenhagen 1979

Sergl HG (1990): Psychologie der Erwachsenenbehandlung. Fortschr Kieferorthop $\underline{51}$, 8-13

Sharma AA, Park JH (2010): Esthetic considerations in interdental papilla: remediation and regeneration. J Esthet Restor Dent 22, 18-28

Shifman A, Laufer BZ, Chweidan H (1998): Posterior bite collapse-revisited. J Oral Rehabil 25, 376-385 
Slade GD (1997): Derivation and validation of a short-form oral health impact profile. Community Dent Oral Epidemiol 25, 284-290

Slade GD, Spencer AJ (1994): Development and evaluation of the Oral Health Impact Profile. Community Dent Health 11, 3-11

Slots J (2004): Systemic antibiotics in periodontics. J Periodontol $\underline{75}, 1553-1565$

Socransky SS, Haffajee AD (2005): Periodontal microbial ecology. Periodontol $2000 \underline{38}, 135-187$

Socransky SS, Haffajee AD, Cugini MA, Smith C, Kent RL, Jr. (1998): Microbial complexes in subgingival plaque. J Clin Periodontol 25, 134-144

Solem RC (2017): Utilizing three-dimensional data in orthodontic practice and research. Orthod Craniofac Res $\underline{20}$ (Supp 1), 114-118

Soskolne WA, Klinger A (2001): The relationship between periodontal diseases and diabetes: an overview. Ann Periodontol $\underline{6}, 91-98$

Stahl SS (1975): The need for orthodontic treatment: a periodontist's point of view. Int Dent J 25, 242-247

Stoltenberg JL, Osborn JB, Pihlstrom BL, Herzberg MC, Aeppli DM, Wolff LF, Fischer GE (1993): Association between cigarette smoking, bacterial pathogens, and periodontal status. J Periodontol $\underline{64}, 1225-1230$

Sun L, Wong HM, McGrath CP (2017): Relationship Between the Severity of Malocclusion and Oral Health Related Quality of Life: A Systematic Review and Meta-analysis. Oral Health Prev Dent $\underline{15}, 503-517$

Taylor GW (2001): Bidirectional interrelationships between diabetes and periodontal diseases: an epidemiologic perspective. Ann Periodontol $\underline{6}, 99-112$

Tessarollo FR, Feldens CA, Closs LQ (2012): The impact of malocclusion on adolescents' dissatisfaction with dental appearance and oral functions. Angle Orthod $\underline{82}, 403-409$

Theilade $E$ (1986): The non-specific theory in microbial etiology of inflammatory periodontal diseases. J Clin Periodontol $\underline{13}$, 905-911

Theilade E, Wright WH, Jensen SB, Loe H (1966): Experimental gingivitis in man. II. A longitudinal clinical and bacteriological investigation. J Periodontal Res 1 , 113

Thilander B (1979): Indications for orthodontic treatment in adults. Eur J Orthod 1, 227-241

Thorstensson H, Hugoson A (1993): Periodontal disease experience in adult long-duration insulin-dependent diabetics. J Clin Periodontol 20, 352-358 
Toma AM, Zhurov A, Playle R, Ong E, Richmond S (2009): Reproducibility of facial soft tissue landmarks on 3D laser-scanned facial images. Orthod Craniofac Res $\underline{12}, 33-42$

Tonetti MS (1998): Cigarette smoking and periodontal diseases: etiology and management of disease. Ann Periodontol $\underline{3}, 88-101$

Tonetti MS, Greenwell H, Kornman KS (2018): Staging and grading of periodontitis: Framework and proposal of a new classification and case definition. J Periodontol $\underline{89}$ (Supp 1), 159-172

Towfighi PP, Brunsvold MA, Storey AT, Arnold RM, Willman DE, McMahan CA (1997): Pathologic migration of anterior teeth in patients with moderate to severe periodontitis. J Periodontol $\underline{68}, 967-972$

Tsuchida S, Satoh M, Takiwaki M, Nomura F (2018): Current Status of Proteomic Technologies for Discovering and Identifying Gingival Crevicular Fluid Biomarkers for Periodontal Disease. Int J Mol Sci 20, 1-11

Tugnait A, Clerehugh V (2001): Gingival recession-its significance and management. J Dent 모, 381-394

van der Linden FP (1975): Theoretical and practical aspects of crowding in the human dentition. Oral Health $\underline{65}, 14-31$

van Dyke TE, Sheilesh D (2005): Risk factors for periodontitis. J Int Acad Periodontol $\underline{7}$, 3-7

van Gastel J, Quirynen M, Teughels W, Carels C (2007): The relationships between malocclusion, fixed orthodontic appliances and periodontal disease. A review of the literature. Aust Orthod J $\underline{23}, 121-129$

Varela-Centelles P, Diz-Iglesias P, Estany-Gestal A, Blanco-Hortas A, BugarinGonzalez R, Seoane-Romero JM, Blanco J (2019): Periodontal awareness and what it actually means: A cross-sectional study. Oral Dis $\underline{25}, 831-838$

Verma D, Garg PK, Dubey AK (2018): Insights into the human oral microbiome. Arch Microbiol 200, 525-540

Vos T, Abajobir AA, Abbafati C, Abbas KM, Abate KH, Abd-Allah F, Abdulle AM, Abebo TA, Abera SF, Aboyans V et al. (2017): Global, regional, and national incidence, prevalence, and years lived with disability for 328 diseases and injuries for 195 countries, 1990-2016: a systematic analysis for the Global Burden of Disease Study 2016. Lancet $\underline{390}, 1211-1259$

Wang SF, Leknes KN, Zimmerman GJ, Sigurdsson TJ, Wikesjo UM, Selvig KA (1995): Intra - and inter-examiner reproducibility in constant force probing. J Clin Periodontol 22, 918-922

Wehrbein H, Wriedt S, Jung BA (2011): Wandel und Innovation in der Kieferorthopädie. Bundesgesundheitsbl $\underline{54}, 1110-1115$ 
Weski DO (1921): Die Röntgendiagnostik bei marginalen Paradentosen. Zahnaerztliche Rundschau 느, 738-739

Wong L, Ryan FS, Christensen LR, Cunningham SJ (2018): Factors influencing satisfaction with the process of orthodontic treatment in adult patients. Am J Orthod Dentofacial Orthop $\underline{153}, 362-370$

Xie Y, Zhao Q, Tan Z, Yang S (2014): Orthodontic treatment in a periodontal patient with pathologic migration of anterior teeth. Am J Orthod Dentofacial Orthop $\underline{145}$, 685-693

Zimmer S, Bizhang M, Seemann R, Barthel CR (2001): Einfluss von Prophylaxeprogrammen auf die Mundhygiene von Erwachsenen und Schulkindern. Gesundheitswesen $\underline{63}, 98-101$ 


\section{Danksagung}

Mein besonderer Dank gilt Herrn Prof. Dr. Philipp Meyer-Marcotty, dem Direktor der Poliklinik für Kieferorthopädie der Universitätsmedizin Göttingen, für die Überlassung des Themas sowie die überaus engagierte Betreuung.

Frau Prof. Dr. Annette Wiegand, der Direktorin der Poliklinik für Präventive Zahnmedizin, Parodontologie und Kariologie der Universitätsmedizin Göttingen, danke ich für die gute Zusammenarbeit.

Frau Dr. Valentina Hraský, Oberärztin der Poliklinik für Präventive Zahnmedizin, Parodontologie und Kariologie der Universitätsmedizin Göttingen, danke ich für die stets unterstützende Hilfe.

Des Weiteren danke ich sowohl den Mitarbeiterinnen der Poliklinik für Kieferorthopädie und der Poliklinik für Präventive Zahnmedizin, Parodontologie und Kariologie der Universitätsmedizin Göttingen für die große Unterstützung bei der Probandenakquise und Durchführung der Studie.

Den Mitarbeiterinnen und Mitarbeitern der Zentralen Serviceeinheit für Medizinische Biometrie und Statistische Bioinformatik am Institut für Medizinische Statistik der Universitätsmedizin Göttingen danke ich für die intensive Beratung.

Ein weiterer Dank gilt Herrn Dr. David Zimmermann für die freundschaftliche Hilfe bei zahlreichen Fragen, welche er jederzeit beantwortete.

Abschließend möchte ich mich sehr herzlich bei allen Patientinnen und Patienten für die Teilnahme an der Studie bedanken. 